\title{
Towards Oka-Cartan theory for algebras of holomorphic functions on coverings of Stein manifolds. II
}

\author{
Alexander Brudnyi and Damir Kinzebulatov
}

\begin{abstract}
We establish basic results of complex function theory within certain algebras of holomorphic functions on coverings of Stein manifolds (such as algebras of Bohr's holomorphic almost periodic functions on tube domains or algebras of all fibrewise bounded holomorphic functions arising, e.g., in the corona problem for $H^{\infty}$ ). In particular, in this context we obtain results on holomorphic extension from complex submanifolds, properties of divisors, corona-type theorems, holomorphic analogues of the Peter-Weyl approximation theorem, Hartogs-type theorems, characterizations of uniqueness sets, etc. Our proofs are based on analogues of Cartan theorems A and B for coherent-type sheaves on maximal ideal spaces of these algebras proved in Part I.
\end{abstract}

\section{Introduction}

In 1930-1950, methods of sheaf theory radically transformed the theory of holomorphic functions of several variables which led to solution of a number of fundamental and long standing problems including problems of holomorphic interpolation, Cousin problems, the Levi problem on characterization of domains of holomorphy, etc. Since then the theory started to play a foundational and unifying role in modern mathematics, with implications for analytic geometry, automorphic forms, Banach algebras, etc. Further development of the theory was motivated, in part, by the problems requiring to study properties of holomorphic functions satisfying additional restrictions (such as uniform boundedness along certain subsets of their domains or certain growth 'at infinity'). In particular, the principal question arose whether the fundamental problems of the function theory of several complex variables can be solved within a proper subclass of the algebra $\mathcal{O}(X)$ of holomorphic

Mathematics Subject Classification (2010): 32A38, 32K99.

Keywords: Oka-Cartan theory, algebras of holomorphic functions, coverings of complex manifolds. 
functions on a Stein manifold $X$. In the present paper we address this question for subalgebras of $\mathcal{O}(X)$ subject to the following definition.

Definition 1.1. A holomorphic function $f$ defined on a regular covering $p: X \rightarrow X_{0}$ of a connected complex manifold $X_{0}$ with a deck transformation group $G$ is called a holomorphic a-function if

(1) $f$ is bounded on subsets $p^{-1}\left(U_{0}\right), U_{0} \Subset X_{0}$, and

(2) for each $x \in X$ the function $G \ni g \mapsto f(g \cdot x)$ belongs to a fixed closed unital subalgebra $\mathfrak{a}:=\mathfrak{a}(G)$ of the algebra $\ell_{\infty}(G)$ of bounded complex functions on $G$ (with pointwise multiplication and sup-norm) that is invariant with respect to the action of $G$ on $\mathfrak{a}$ by right translations:

$$
u \in \mathfrak{a}, \quad g \in G \quad \Longrightarrow \quad R_{g} u \in \mathfrak{a},
$$

where $R_{g}(u)(h):=u(h g), h \in G$.

We endow the subalgebra $\mathcal{O}_{\mathfrak{a}}(X) \subset \mathcal{O}(X)$ of holomorphic $\mathfrak{a}$-functions with the Fréchet topology of uniform convergence on subsets $p^{-1}\left(U_{0}\right), U_{0} \Subset X_{0}$.

The model examples of algebras $\mathcal{O}_{\mathfrak{a}}(X)$ are:

(1) Bohr's holomorphic almost periodic functions on a tube domain $T \subset \mathbb{C}^{n}$, see Example 1.2 below;

(2) all fibrewise bounded holomorphic functions on $X$, see Example 1.4(1) below. (If $X_{0}$ is a compact complex manifold, then this algebra coincides with algebra $H^{\infty}(X)$ of bounded holomorphic functions on $\left.X\right)$.

See Section 3 for other examples.

In [15] we derived analogues of Cartan's theorems A and B for coherent-type sheaves on the fibrewise compactification $c_{\mathfrak{a}} X$ of the covering $X$ of a Stein manifold $X_{0}$, a topological space having certain features of a complex manifold (see Section 2 for details). This allows us to transfer in a systematic manner most of the significant results of the classical theory of holomorphic functions on Stein manifolds to holomorphic functions in algebras $\mathcal{O}_{\mathfrak{a}}(X)$. In particular, in the present paper we establish:

- results on holomorphic interpolation within algebra $\mathcal{O}_{\mathfrak{a}}(X)$ over complex a-submanifolds (i.e., complex submanifolds of $X$ determined by holomorphic a-functions) (Subsection 2.2),

- tubular neighbourhood theorem for complex a-submanifolds (Subsection 2.2),

- properties of holomorphic line a-bundles and their divisors (Subsection 2.3),

- characterization of uniqueness sets for functions in $\mathcal{O}_{\mathfrak{a}}(X)$ (Subsection 2.4),

- Leray integral representation formulas and Hartogs theorems for functions in $\mathcal{O}_{\mathfrak{a}}(X)$ (Subsection 2.5),

- holomorphic Peter-Weyl theorems for $\mathcal{O}_{\mathfrak{a}}(X)$ (Subsection 2.5),

- Cartan's theorems A and B for coherent-type sheaves on complex a-submanifolds (Subsection 5.4), 
- Dolbeault isomorphisms on complex a-submanifolds (Subsection 5.5),

- corona theorems for algebras $\mathcal{O}_{\mathfrak{a}}(X)$ (Subsection 5.6).

Note that the classical proof of Cartan theorems A and B on complex manifolds does not work in our case, in particular, because of absence of the Oka coherence lemma, and since the fibre $\hat{G}_{\mathfrak{a}}$ of the covering $c_{\mathfrak{a}} X \rightarrow X_{0}$ being an arbitrary compact Hausdorff space does not admit open covers by contractible sets as required for the proof of the classical Cartan lemma. Instead, we develop a new approach to the proof of Cartan theorems A and B, where we use some results from [42] and [41].

Example 1.2 (Holomorphic almost periodic functions). The theory of almost periodic functions was created in the $1920 \mathrm{~s}$ by $\mathrm{H}$. Bohr and nowadays is widely used in various areas of mathematics including number theory, harmonic analysis, differential equations (e.g., KdV equation), etc.

Let us recall the S. Bochner (equivalent) definition of almost periodicity: a function $f \in \mathcal{O}(T)$ on a tube domain $T=\mathbb{R}^{n}+i \Omega \subset \mathbb{C}^{n}, \Omega \subset \mathbb{R}^{n}$ is open and convex, is called holomorphic almost periodic if the family of its translates $\{z \mapsto f(z+s), z \in T\}_{s \in \mathbb{R}^{n}}$ is relatively compact in the topology of uniform convergence on tube subdomains $T^{\prime}=\mathbb{R}^{n}+i \Omega^{\prime}, \Omega^{\prime} \Subset \Omega$. The principal result of Bohr's theory (see [2]) is the approximation theorem which states that every holomorphic almost periodic function is the uniform limit (on tube subdomains $T^{\prime}$ of $T$ ) of exponential polynomials

$$
z \mapsto \sum_{k=1}^{m} c_{k} e^{i\left\langle z, \lambda_{k}\right\rangle}, \quad z \in T, \quad c_{k} \in \mathbb{C}, \quad \lambda_{k} \in \mathbb{R}^{n},
$$

where $\left\langle\cdot, \lambda_{k}\right\rangle$ is the Hermitian inner product on $\mathbb{C}^{n}$.

The classical approach to study of holomorphic almost periodic functions exploits the fact that $T$ is the trivial bundle with base $\Omega$ and fibre $\mathbb{R}^{n}$ (e.g., as in the characterization of almost periodic functions in terms of their Jessen functions defined on $\Omega$, see [51], [43], [38], [48], [20], [52] and references therein). In our approach, we consider $T$ as a regular covering $p: T \rightarrow T_{0}\left(:=p(T) \subset \mathbb{C}^{n}\right)$ with the deck transformation group $\mathbb{Z}^{n}$, where $p(z):=\left(e^{i z_{1}}, \ldots, e^{i z_{n}}\right), z=\left(z_{1}, \ldots, z_{n}\right) \in T$ (if $n=1$, then this is a complex strip covering an annulus in $\mathbb{C}$ ), and obtain:

Theorem 1.3. A function $f \in \mathcal{O}(T)$ is almost periodic if and only if $f \in \mathcal{O}_{A P}(T)$.

(Here $A P=A P\left(\mathbb{Z}^{n}\right)$ is the algebra of von Neumann's almost periodic functions on group $\mathbb{Z}^{n}$, see definition in Example 3.1(2) below.) This result enables us to regard holomorphic almost periodic functions on $T$ as:

(a) holomorphic sections of a certain holomorphic Banach vector bundle on $T_{0}$;

(b) holomorphic-like functions on the fibrewise Bohr compactification $c_{A P} T$ of the covering $p: T \rightarrow T_{0}$.

As a result, we can apply the methods of multidimensional complex function theory (in particular, analytic sheaf theory and Banach-valued complex analysis) 
to study holomorphic almost periodic functions. In particular, even in this classical setting, we obtain new results on holomorphic almost periodic interpolation, recovery of almost periodicity of a holomorphic function from that for its trace to a real periodic hypersurface, etc. We also show that some results known for holomorphic almost periodic functions are, in fact, valid for a general algebra $\mathcal{O}_{\mathfrak{a}}(X)$.

It is interesting to note that already in his monograph [2], H. Bohr uses equally often the aforementioned "trivial fibre bundle" and "regular covering" points of view on a complex strip. We mention also that the Bohr compactification of a tube domain $\mathbb{R}^{n}+i \Omega$ in the form $b \mathbb{R}^{n}+i \Omega$, where $b \mathbb{R}^{n}$ is the Bohr compactification of group $\mathbb{R}^{n}$, was used earlier in [18], [19], [31].

Example 1.4. (1) By definition, every $\mathcal{O}_{\mathfrak{a}}(X) \subset \mathcal{O}_{\ell_{\infty}(G)}(X)$; here $G$ is the deck transformation group of covering $p: X \rightarrow X_{0}$.

Algebra $\mathcal{O}_{\ell_{\infty}(G)}(X)$ arises, e.g., in study of holomorphic $L^{2}$-functions on coverings of pseudoconvex manifolds [32], [6], [9], [40], Caratheodory hyperbolicity (the Liouville property) of $X[45],[44]$, corona-type problems for bounded holomorphic functions on $X$ [5]. Earlier, some methods similar to those developed in the present paper were elaborated for algebra $\mathcal{O}_{\ell_{\infty}(G)}(X)$ in $[5]-[8],[10]$ in connection with corona-type problems for some subalgebras of bounded holomorphic functions on coverings of bordered Riemann surfaces, Hartogs-type theorems, integral representation of holomorphic functions of slow growth on coverings of Stein manifolds, etc.

A confirmation of potential productivity of the sheaf-theoretic approach to corona problem for $H^{\infty}$ comes from the recent papers [11], [12] on Banach-valued holomorphic functions on the unit disk $\mathbb{D} \subset \mathbb{C}$ having relatively compact images.

(2) Let $\mathfrak{a}:=c(G) \subset \ell_{\infty}(G)$ (with card $G=\infty$ ) be the subalgebra of bounded complex functions on $G$ that admit continuous extensions to the one-point compactification of $G$. Then $\mathcal{O}_{c}(X)$ consists of holomorphic functions that have fibrewise limits at 'infinity'.

For other examples of algebras $\mathfrak{a}$ and $\mathcal{O}_{\mathfrak{a}}(X)$ see Subsections 3.1 and 3.2.

In the formulation of our main results we use the following definitions.

Assume that $d$ is a path metric on $X$ defined by the pullback to $X$ of a (smooth) hermitian metric on $X_{0}$.

A function $f \in C(X)$ is called a continuous $\mathfrak{a}$-function if it is bounded and uniformly continuous with respect to metric $d$ on subsets $p^{-1}\left(U_{0}\right), U_{0} \Subset X_{0}$, and is such that for each $x \in X$ the function $G \ni g \mapsto f(g \cdot x)$ belongs to $\mathfrak{a}$.

We denote by $C_{\mathfrak{a}}(X)$ the algebra of continuous a-functions on $X$. It is easily seen that $C_{\mathfrak{a}}(X)$ does not depend on the choice of the hermitian metric on $X_{0}$ and $C_{\mathfrak{a}}(X) \cap \mathcal{O}(X)=\mathcal{O}_{\mathfrak{a}}(X)$.

If $D_{0} \Subset X_{0}$ is a subdomain, we set $D:=p^{-1}\left(D_{0}\right) \subset X$ and define $C_{\mathfrak{a}}(\bar{D})$ to be the subalgebra of complex functions $f$ on $\bar{D}$ (the closure of $D$ ) that are bounded and uniformly continuous with respect to path metric $d$ and such that for each $x \in \bar{D}_{0}$ functions $G \ni g \mapsto f(g \cdot x)$ belong to $\mathfrak{a}$.

Let $\mathcal{L}\left(B_{1}, B_{2}\right)$ denote the space of bounded linear operators $B_{1} \rightarrow B_{2}$ between complex Banach spaces $B_{1}$ and $B_{2}$. 
Acknowledgement. We thank Professors T. Bloom, L. Lempert, T. Ohsawa, R. Shafikov and Y.-T. Siu for their interest to this work.

\section{Main results}

\subsection{Analogues of Cartan theorems A and B}

Our approach is based on analogues of the Cartan theorems A and B for coherenttype sheaves on the fibrewise compactification $c_{\mathfrak{a}} X$ of covering $p: X \rightarrow X_{0}$. We briefly describe its construction postponing details till Section 5 (see also [15]).

Let $M_{\mathfrak{a}}$ denote the maximal ideal space of algebra $\mathfrak{a}$, i.e., the space of all characters $\mathfrak{a} \rightarrow \mathbb{C}$ endowed with weak ${ }^{*}$ topology (of $\mathfrak{a}^{*}$ ). The space $M_{\mathfrak{a}}$ is compact Hausdorff and every element $f$ of $\mathfrak{a}$ determines a function $\hat{f} \in C\left(M_{\mathfrak{a}}\right)$ by the formula

$$
\hat{f}(\eta):=\eta(f), \quad \eta \in M_{\mathfrak{a}} .
$$

Since algebra $\mathfrak{a}$ is uniform (i.e., $\left\|f^{2}\right\|=\|f\|^{2}$ ) and hence is semi-simple, the homomorphism ^ $: \mathfrak{a} \rightarrow C\left(M_{\mathfrak{a}}\right)$ (called the Gelfand transform) is an isometric embedding (see, e.g., [27]). We have a continuous map $j=j_{\mathfrak{a}}: G \rightarrow M_{\mathfrak{a}}$ defined by associating to each point in $G$ its point evaluation homomorphism in $M_{\mathfrak{a}}$. This map is an injection if and only if algebra $\mathfrak{a}$ separates points of $G$.

Let $\hat{G}_{\mathfrak{a}}$ denote the closure of $j(G)$ in $M_{\mathfrak{a}}$ (also a compact Hausdorff space). If algebra $\mathfrak{a}$ is self-adjoint with respect to complex conjugation, then $\mathfrak{a} \cong C\left(M_{\mathfrak{a}}\right)$ and hence $\hat{G}_{\mathfrak{a}}=M_{\mathfrak{a}}$. In a standard way the action of group $G$ on itself by right multiplication determines the right action of $G$ on $M_{\mathfrak{a}}$, so that $\hat{G}_{\mathfrak{a}}$ is invariant with respect to this action.

Definition 2.1. The fibrewise compactification $\bar{p}: c_{\mathfrak{a}} X \rightarrow X_{0}$ is defined to be the fibre bundle with fibre $\hat{G}_{\mathfrak{a}}$ associated to the regular covering $p: X \rightarrow X_{0}$ (regarded as a principal bundle with fibre $G$ ).

There exists a continuous map

$$
\iota=\iota_{\mathfrak{a}}: X \longrightarrow c_{\mathfrak{a}} X
$$

induced by the equivariant map $j$. Clearly, $\iota(X)$ is dense in $c_{\mathfrak{a}} X$. If $\mathfrak{a}$ separates points of $G$, then $\iota$ is an injection.

Definition 2.2. A function $f \in C\left(c_{\mathfrak{a}} X\right)$ is called holomorphic if its pullback $\iota^{*} f$ is holomorphic on $X$. The algebra of functions holomorphic on $c_{\mathfrak{a}} X$ is denoted by $\mathcal{O}\left(c_{\mathfrak{a}} X\right)$.

For a subalgebra $\mathfrak{a} \subset \ell_{\infty}(G)$ we have a monomorphism $\mathcal{O}_{\mathfrak{a}}(X) \hookrightarrow \mathcal{O}\left(c_{\mathfrak{a}} X\right)$ (see Proposition 5.1 below) which is an isomorphism if $\mathfrak{a}$ is self-adjoint (in this case we can work with algebra $\mathcal{O}\left(c_{\mathfrak{a}} X\right)$ instead of $\left.\mathcal{O}_{\mathfrak{a}}(X)\right)$. See [15] for the description of the complex-analytic structure on $c_{\mathfrak{a}} X$. 
Analogously to Definition 2.2, we define holomorphic functions on open subsets of $c_{\mathfrak{a}} X$ and thus obtain the structure sheaf $\mathcal{O}:=\mathcal{O}_{c_{\mathfrak{a}} X}$ of germs of holomorphic functions on $c_{\mathfrak{a}} X$. Now, a coherent sheaf $\mathcal{A}$ on $c_{\mathfrak{a}} X$ is a sheaf of modules over $\mathcal{O}$ such that every point in $c_{\mathfrak{a}} X$ has a neighbourhood $U$ over which, for every $N \geq 1$, there is a free resolution of $\mathcal{A}$ of length $N$, i.e., an exact sequence of sheaves of modules of the form

$$
\left.\left.\left.\left.\mathcal{O}^{m_{N}}\right|_{U} \stackrel{\varphi_{N-1}}{\longrightarrow} \cdots \stackrel{\varphi_{2}}{\longrightarrow} \mathcal{O}^{m_{2}}\right|_{U} \stackrel{\varphi_{1}}{\longrightarrow} \mathcal{O}^{m_{1}}\right|_{U} \stackrel{\varphi_{0}}{\longrightarrow} \mathcal{A}\right|_{U} \longrightarrow 0
$$

(here $\varphi_{i}, 0 \leq i \leq N-1$, are homomorphisms of sheaves of modules). If $X=X_{0}$ and $p=\mathrm{Id}$, then this definition gives the classical definition of a coherent sheaf on a complex manifold $X_{0}$.

Let $X_{0}$ be a Stein manifold, $\mathcal{A}$ a coherent sheaf on $c_{\mathfrak{a}} X$.

Theorem 2.3 ([15]). Each stalk ${ }_{x} \mathcal{A}\left(x \in c_{\mathfrak{a}} X\right)$ is generated by global sections of $\mathcal{A}$ over $c_{\mathfrak{a}} X$ as an ${ }_{x} \mathcal{O}$-module ( "Cartan-type Theorem $A$ ").

Theorem 2.4 ([15]). Čech cohomology groups $H^{i}\left(c_{\mathfrak{a}} X, \mathcal{A}\right)=0$ for all $i \geq 1$ ( "Cartan-type Theorem B").

The collection of open subsets of $X$ of the form $V=\iota^{-1}(U)$, where $U \subset c_{\mathfrak{a}} X$ is open, determines a topology on $X$, denoted by $\mathcal{T}_{\mathfrak{a}}$, which is Hausdorff if and only if $\mathfrak{a}$ separates points of $G$. (A basis of $\mathcal{T}_{\mathfrak{a}}$ consists of interiors of sublevel sets of functions in $C_{\mathfrak{a}}(X)$.) If algebra $\mathfrak{a}$ is self-adjoint, we define spaces of continuous and holomorphic a-functions on $V=\iota^{-1}(U) \in \mathcal{T}_{\mathfrak{a}}$ by

$$
C_{\mathfrak{a}}(V):=\iota^{*} C(U), \quad \mathcal{O}_{\mathfrak{a}}(V):=\iota^{*} \mathcal{O}(U) .
$$

Thus, if $V=X, U=c_{\mathfrak{a}} X$, we obtain algebras $C_{\mathfrak{a}}(X), \mathcal{O}_{\mathfrak{a}}(X)$ as defined in Section 1 and Definition 1.1 (for holomorphic functions this is proved in Proposition $2.3(2)$ of [15]; for continuous functions the argument is similar to the one in the proof of the latter proposition).

For subsets $V, W \in \mathcal{T}_{\mathfrak{a}}$ we denote

$$
\mathcal{O}_{\mathfrak{a}}(V, W):=\left\{f \in C(V, W): f^{*} h \in \mathcal{O}_{\mathfrak{a}}(V) \text { for all } h \in \mathcal{O}_{\mathfrak{a}}(W)\right\} .
$$

In Subsections 2.2, 2.3 and 2.4 we assume that algebra $\mathfrak{a}$ is self-adjoint.

\subsection{Complex a-submanifolds and their properties}

We now formulate the results on complex submanifolds determined by holomorphic $\mathfrak{a}$-functions, the corresponding tubular neighbourhood theorem, and the result on interpolation within $\mathcal{O}_{\mathfrak{a}}(X)$. We will need:

Definition 2.5. An open cover $\mathcal{V}$ of $X$ is said to be of class $\left(\mathcal{T}_{\mathfrak{a}}\right)$ if it is the pullback by $\iota$ of an open cover of $c_{\mathfrak{a}} X$ (e.g., $\mathcal{V}=p^{-1}\left(\mathcal{V}_{0}\right)$, where $\mathcal{V}_{0}$ is an open cover of $X_{0}$ is of class $\left.\left(\mathcal{T}_{\mathfrak{a}}\right)\right)$. 
It is easy to see that any open cover of $X$ by sets in $\mathcal{T}_{\mathfrak{a}}$ is a subcover of an open cover of $X$ of class $\left(\mathcal{T}_{\mathfrak{a}}\right)$.

Definition 2.6. A closed subset $Z \subset X$ is called a complex a-submanifold of codimension $k \leq n:=\operatorname{dim}_{\mathbb{C}} X_{0}$ if there exists an open cover $\mathcal{V}$ of $X$ of class $\left(\mathcal{T}_{\mathfrak{a}}\right)$ such that for each $V \in \mathcal{V}$ the closure of $p(V)$ is compact and contained in a coordinate chart on $X_{0}$ and either $V \cap Z=\emptyset$ or there are functions $h_{1}, \ldots, h_{k} \in$ $\mathcal{O}_{\mathfrak{a}}(V)$ that satisfy:

(1) $Z \cap V=\left\{x \in V: h_{1}(x)=\cdots=h_{k}(x)=0\right\}$;

(2) the maximum of moduli of determinants of all $k \times k$ submatrices of the Jacobian matrix of the map $x \mapsto\left(h_{1}(x), \ldots, h_{k}(x)\right)$ with respect to local coordinates on $V$ pulled back from a coordinate chart on $X_{0}$ containing the closure of $p(V)$ is uniformly bounded away from zero on $Z \cap V$.

Some examples of complex $\mathfrak{a}$-submanifolds are given in Subsection 3.4 below.

We have analogues of Cartan-type theorems 2.3 and 2.4 on complex a-submanifolds, see Subsection 5.3 and Theorems 5.11, 5.12 below.

Theorem 2.7 (Characterization of complex a-submanifolds). Suppose that $X_{0}$ is a Stein manifold. Then a closed subset $Z \subset X$ is a complex a-submanifold of codimension $k \leq n$ if and only if there exist an at most countable collection of globally defined functions $f_{i} \in \mathcal{O}_{\mathfrak{a}}(X), i \in I$, and an open cover $\mathcal{V}$ of $X$ of class $\left(\mathcal{T}_{\mathfrak{a}}\right)$ such that

(i) $Z=\left\{x \in X: f_{i}(x)=0\right.$ for all $\left.i \in I\right\}$,

(ii) for each $V \in \mathcal{V}$ the closure $p(V)$ is compact and contained in a coordinate chart on $X_{0}$, and either $V \cap Z=\emptyset$ or there are functions $f_{i_{1}}, \ldots f_{i_{k}}$ such that $Z \cap V=\left\{x \in V: f_{i_{1}}=\cdots=f_{i_{k}}=0\right\}$ and the maximum of moduli of determinants of all $k \times k$ submatrices of the Jacobian matrix of the map $x \mapsto\left(f_{i_{1}}(x), \ldots, f_{i_{k}}(x)\right)$ with respect to local coordinates on $V$ pulled back from a coordinate chart on $X_{0}$ containing the closure of $p(V)$ is uniformly bounded away from zero on $Z \cap V$.

We prove Theorem 2.7 in Section 9.

Definition 2.8. A function $f \in \mathcal{O}(Z)$ on a complex a-submanifold $Z \subset X$ is called a holomorphic a-function if it admits an extension to a function in $C_{\mathfrak{a}}(X)$.

The subalgebra of holomorphic $\mathfrak{a}$-functions on $Z$ is denoted by $\mathcal{O}_{\mathfrak{a}}(Z)$.

Alternatively, subalgebra $\mathcal{O}_{\mathfrak{a}}(Z)$ can be defined in terms of a-currents, see Subsection 4.1 .

We have the following analogue of the classical tubular neighbourhood theorem:

Theorem 2.9. Let $X_{0}$ be a Stein manifold, $Z \subset X$ be a complex $\mathfrak{a}$-submanifold. Then there exist an open in topology $\mathcal{T}_{\mathfrak{a}}$ neighbourhood $\Omega \subset X$ of $Z$ and a family of maps $h_{t} \in \mathcal{O}_{\mathfrak{a}}(\Omega, \Omega)$ continuously depending on $t \in[0,1]$ such that

$$
\left.h_{t}\right|_{Z}=\operatorname{Id}_{Z} \quad \text { for all } t \in[0,1], \quad h_{0}=\operatorname{Id}_{\Omega} \quad \text { and } \quad h_{1}(\Omega)=Z .
$$


Theorem 2.9 gives a linear extension operator $h_{1}^{*}: \mathcal{O}_{\mathfrak{a}}(Z) \rightarrow \mathcal{O}_{\mathfrak{a}}(\Omega), f \mapsto h_{1}^{*} f$. Using Theorem 2.4 we prove the following interpolation result.

Theorem 2.10. Suppose $X_{0}$ is a Stein manifold, $Z \subset X$ is a complex a-submanifold, and $f \in \mathcal{O}_{\mathfrak{a}}(Z)$. Then there is $F \in \mathcal{O}_{\mathfrak{a}}(X)$ such that $\left.F\right|_{Z}=f$.

We prove Theorems 2.9 and 2.10 in Section 9.

Example 2.11. Suppose that $Z_{1}, Z_{2} \subset T:=\mathbb{R}^{n}+i \Omega \subset \mathbb{C}^{n}$ (where $\Omega \subset \mathbb{R}^{n}$ is open and convex) are non-intersecting smooth complex hypersurfaces that are periodic, possibly with different periods, with respect to the usual action of $\mathbb{R}^{n}$ on $T$ by translations. Suppose also that the Euclidean distance $\operatorname{dist}\left(Z_{1}, Z_{2}\right)>0$. Let $f_{1} \in \mathcal{O}\left(Z_{1}\right), f_{2} \in \mathcal{O}\left(Z_{2}\right)$ be holomorphic functions periodic with respect to these periods. The union $Z_{1} \cup Z_{2}$ is a complex almost periodic submanifold of $T$ in the sense of Definition 2.6 (cf. Example 1.2), and so by Theorem 2.10 there is a holomorphic almost periodic function $F \in \mathcal{O}_{A P}(T)$ such that $\left.F\right|_{Z_{i}}=f_{i}, i=1,2$.

\subsection{Holomorphic line a-bundles and their divisors}

This subsection describes our results on $\mathfrak{a}$-divisors.

Let $Z \subset X$ be a complex submanifold. Recall that a continuous line bundle $L$ on $Z$ is given by an open cover $\left\{U_{\alpha}\right\}$ of $Z$ and nowhere zero functions $d_{\alpha \beta} \in$ $C\left(U_{\alpha} \cap U_{\beta}\right)$ (where $d_{\alpha \beta}:=1$ if $U_{\alpha} \cap U_{\beta}=\varnothing$ ) satisfying the 1-cocycle conditions:

$$
\begin{aligned}
\forall \alpha, \beta \quad d_{\alpha \beta}=d_{\beta \alpha}^{-1} & \text { on } U_{\alpha} \cap U_{\beta}, \\
\forall \alpha, \beta, \gamma \quad d_{\alpha \beta} d_{\beta \gamma} d_{\gamma \alpha}=1 & \text { on } U_{\alpha} \cap U_{\beta} \cap U_{\gamma} \neq \varnothing .
\end{aligned}
$$

If all $d_{\alpha \beta} \in \mathcal{O}\left(U_{\alpha} \cap U_{\beta}\right)$, then $L$ is called a holomorphic line bundle.

In a standard way one defines continuous and holomorphic line bundles morphisms (see, e.g., [37]). The categories of continuous and holomorphic line bundles on $Z$ are denoted by $\mathcal{L}^{c}(Z)$ and $\mathcal{L}(Z)$, respectively.

An effective (Cartier) divisor $E$ on $Z$ is given by an open cover $\left\{U_{\alpha}\right\}$ of $Z$ and not identically zero on open subsets of $U_{\alpha}$ functions $f_{\alpha} \in \mathcal{O}\left(U_{\alpha}\right)$ such that

$$
\forall \alpha, \beta \quad f_{\alpha}=d_{\alpha \beta} f_{\beta} \text { on } U_{\alpha} \cap U_{\beta} \text { for some } d_{\alpha \beta} \in \mathcal{O}\left(U_{\alpha} \cap U_{\beta}, \mathbb{C} \backslash\{0\}\right) .
$$

Clearly, holomorphic 1-cocycle $\left\{d_{\alpha \beta}\right\}$ determines a holomorphic line bundle denoted by $L_{E}$.

The collection of effective divisors on $Z$ is denoted by $\operatorname{Div}(Z)$.

Divisors $E=\left\{\left(U_{\alpha}, f_{\alpha}\right)\right\}$ and $E^{\prime}=\left\{\left(V_{\beta}, g_{\beta}\right)\right\}$ in $\operatorname{Div}(Z)$ are said to be equivalent (in $\operatorname{Div}(Z)$ ) if there exists a refinement $\left\{W_{\gamma}\right\}$ of both covers $\left\{U_{\alpha}\right\}$ and $\left\{V_{\beta}\right\}$ and nowhere zero functions $p_{\gamma} \in \mathcal{O}\left(W_{\gamma}\right)$ such that

$$
\left.f_{\alpha}\right|_{W_{\gamma}}=\left.p_{\gamma} \cdot g_{\beta}\right|_{W_{\gamma}} \quad \text { for } W_{\gamma} \subset U_{\alpha} \cap V_{\beta} .
$$

If divisors $E, E^{\prime}$ are equivalent, then their line bundles $L_{E}, L_{E^{\prime}}$ are isomorphic.

Now, let $Z \subset X$ be either a complex $\mathfrak{a}$-submanifold or $X$ itself. 
Definition 2.12. An open cover of $Z$ is said to be of class $\left(\mathcal{T}_{\mathfrak{a}}\right)$ if it is the pullback by $\iota$ of an open cover of the closure of $\iota(Z)$ in $c_{\mathfrak{a}} X$ (cf. Definition 2.5).

Definition 2.13. A continuous line bundle $L$ on $Z$ is called an $\mathfrak{a}$-bundle if, in its definition (see (2.4) and (2.5)),

(1) $\left\{U_{\alpha}\right\}$ is of class $\left(\mathcal{T}_{\mathfrak{a}}\right)$,

(2) $\forall \alpha, \beta \quad d_{\alpha \beta} \in C_{\mathfrak{a}}\left(U_{\alpha} \cap U_{\beta}\right)$.

If all $d_{\alpha \beta} \in \mathcal{O}_{\mathfrak{a}}\left(U_{\alpha} \cap U_{\beta}\right)$, then $L$ is called a holomorphic line $\mathfrak{a}$-bundle.

The categories of continuous and holomorphic line a-bundles on $Z$ are denoted by $\mathcal{L}_{\mathfrak{a}}^{c}(Z)$ and $\mathcal{L}_{\mathfrak{a}}(Z)$, respectively.

Definition 2.14. A divisor $E \in \operatorname{Div}(Z)$ is called an effective a-divisor if, in its definition (see (2.6)),

(1) $\left\{U_{\alpha}\right\}$ is of class $\left(\mathcal{T}_{\mathfrak{a}}\right)$,

(2) $\forall \alpha \quad f_{\alpha} \in \mathcal{O}_{\mathfrak{a}}\left(U_{\alpha}\right)$,

(3) $\forall \alpha, \beta \quad f_{\alpha}=d_{\alpha \beta} f_{\beta}$ on $U_{\alpha} \cap U_{\beta}$ for some $d_{\alpha \beta} \in \mathcal{O}_{\mathfrak{a}}\left(U_{\alpha} \cap U_{\beta}\right)$.

The collection of $\mathfrak{a}$-divisors is denoted by $\operatorname{Div}_{\mathfrak{a}}(Z)$.

By the definition the line bundle $L_{E}$ of an a-divisor $E$ is a holomorphic line $\mathfrak{a}$-bundle.

Definition 2.15. a-divisors $E=\left\{\left(U_{\alpha}, f_{\alpha}\right)\right\}$ and $E^{\prime}=\left\{\left(V_{\beta}, g_{\beta}\right)\right\}$ are said to be $\mathfrak{a}$-equivalent if, in the above definition of equivalence in $\operatorname{Div}(Z)$ (see $(2.7)$ ),

(1) $\left\{W_{\gamma}\right\}$ is of class $\left(\mathcal{T}_{\mathfrak{a}}\right)$,

(2) $\forall \gamma p_{\gamma}, p_{\gamma}^{-1} \in \mathcal{O}_{\mathfrak{a}}\left(W_{\gamma}\right)$.

If divisors $E$ and $E^{\prime}$ are a-equivalent, then their line bundles $L_{E}$ and $L_{E^{\prime}}$ are isomorphic in $\mathcal{L}_{\mathfrak{a}}(Z)$.

For some algebras $\mathfrak{a}$ (e.g., algebras of holomorphic almost periodic functions, see Example 1.2 and Subsection 3.2) a-divisors can be equivalently defined in terms of their currents of integration, see Subsection 4.3.

The basic example of an $\mathfrak{a}$-divisor is divisor $E_{f}$ of a function $f \in \mathcal{O}_{\mathfrak{a}}(Z)$, called an $\mathfrak{a}$-principal divisor. There are, however, divisors in $\operatorname{Div}_{\mathfrak{a}}(Z)$ that are not $\mathfrak{a}$ principal (see Subsection $3.4(4)$ ); because the Čech cohomology group $H^{2}(\overline{\iota(Z)}, \mathbb{Z}$ ), where $\overline{\iota(Z)}$ is the closure of $\iota(Z)$ in $c_{\mathfrak{a}} X$, whose elements measure deviations of $\mathfrak{a}$-divisors on $X$ from being $\mathfrak{a}$-principal is in general non-trivial (see the proof of Theorem 2.20 for details). This naturally leads to the following question, first considered in [21] in the case of holomorphic almost periodic functions:

Suppose that $X_{0}$ is Stein and $H^{2}(Z, \mathbb{Z})=0$. Does there exist a class of functions $\mathfrak{C}_{\mathfrak{a}} \subset \mathcal{O}_{\ell^{\infty}}(Z), \ell_{\infty}:=\ell_{\infty}(G)$, such that for each function from $\mathfrak{C}_{\mathfrak{a}}$ its divisor is equivalent $\left(\right.$ in $\operatorname{Div}_{\ell_{\infty}}(Z)$ ) to a divisor in $\operatorname{Div}_{\mathfrak{a}}(Z)$, and conversely, every divisor in $\operatorname{Div}_{\mathfrak{a}}(Z)$ is equivalent to a principal divisor determined by a function in $\mathfrak{C}_{\mathfrak{a}}$ ? 
If $Z=X=\{z \in \mathbb{C}: a<\operatorname{Im}(z)<b\}$ and $\mathfrak{a}=A P(\mathbb{Z})$ (see Example 1.2), then it was established in [21] that the class

$$
\mathfrak{C}_{A P}=\left\{f \in \mathcal{O}(Z):|f| \in C_{A P}(Z)\right\}
$$

satisfies this property. (The proof in [21] uses some properties of almost periodic currents.) In Proposition 2.19 we extend this result to a-divisors defined on certain one-dimensional complex manifolds $Z$. In turn, the results in [19] show that for the algebra of holomorphic almost periodic functions $\mathcal{O}_{A P}(Z)$ on a tube domain $Z \subset \mathbb{C}^{n}$ with $n>1$ (see Example 1.2) the functions in $\mathfrak{C}_{A P}$ determine only a proper subclass of almost periodic (i.e., $A P$-) divisors and provide a complete description of this subclass. Using our sheaf-theoretic approach we extend this result in Theorem 2.18 and Proposition 2.19 below.

To formulate the results we require:

Definition 2.16. A line bundle $L \in \mathcal{L}_{\mathfrak{a}}(Z)$ is called $\mathfrak{a}$-semi-trivial if there exists an isomorphism $\psi$ in category $\mathcal{L}_{\ell_{\infty}}(Z)$ of $L$ onto the trivial line bundle $L_{0} \in \mathcal{L}_{\ell_{\infty}}(Z)$ such that $|\psi|^{2}:=\psi \otimes \bar{\psi}$ is an isomorphism in category $\mathcal{L}_{\mathfrak{a}}^{c}(Z)$ of $L \otimes \bar{L}$ onto $L_{0} \otimes \bar{L}_{0}$.

(Here $\bar{L}$ is the bundle defined by complex conjugation of fibres of $L$.)

This definition is related to the question raised above via the following result (where we do not assume that $X_{0}$ is Stein).

Theorem 2.17. If the line bundle $L_{E}$ of an $\mathfrak{a}$-divisor $E$ is a-semi-trivial, then $E$ is $\ell_{\infty}$-equivalent to divisor $E_{f} \in \operatorname{Div}(Z)$ of a function $f \in \mathcal{O}(Z)$ with $|f| \in C_{\mathfrak{a}}(Z)$.

Suppose that $\mathfrak{a}$ is such that $\hat{G}_{\mathfrak{a}}$ is a compact topological group and $j(G) \subset \hat{G}_{\mathfrak{a}}$ is a dense subgroup. Then for $Z=X$ the converse holds:

If $E \in \operatorname{Div}_{\mathfrak{a}}(X)$ is $\ell_{\infty}$-equivalent to $E_{f} \in \operatorname{Div}(X)$ with $|f| \in C_{\mathfrak{a}}(X)$, then $L_{E}$ is a-semi-trivial.

The second statement of the theorem is valid, e.g., for $\mathfrak{a}=A P(G)$, the algebra of von Neumann almost periodic functions on the deck transformation group $G$, see Example 3.1 (2) below. In this case $\hat{G}_{\mathfrak{a}}:=b G$, the Bohr compactification of $G$.

Now, we characterize the class of $\mathfrak{a}$-semi-trivial holomorphic line $\mathfrak{a}$-bundles.

Theorem 2.18. Suppose $X_{0}$ is a Stein manifold. A line bundle $L \in \mathcal{L}_{\mathfrak{a}}(Z)$ is $\mathfrak{a}$-semi-trivial if and only if

(1) $L$ is isomorphic in category $\mathcal{L}_{\mathfrak{a}}(Z)$ to a discrete line a-bundle $L^{\prime}$ (i.e., a bundle determined by a locally constant cocycle), and

(2) $L^{\prime}$ is trivial in the category of discrete line bundles on $Z$.

The argument in the proof of Theorem 2.18 implies that if the line bundle $L_{E}$ of an $\mathfrak{a}$-divisor $E$ satisfies condition (1) only, then the current of integration associated with $E$ (see Subsection 4.3 ) coincides with $\frac{i}{\pi} \partial \bar{\partial} \log h$, where $h$ is a nonnegative continuous plurisubharmonic $\mathfrak{a}$-function on $Z$. 
Proposition 2.19. Suppose $X_{0}$ is a Stein manifold and $Z \subset X$ is one-dimensional. Then for a line bundle $L \in \mathcal{L}_{\mathfrak{a}}(Z)$ condition (1) of Theorem 2.18 is satisfied. If also $H^{1}(Z, \mathbb{C})=0$, then condition (2) is satisfied as well.

In particular, conditions (1) and (2) of Theorem 2.18 are satisfied if $Z=X$ is the universal covering of a non-compact Riemann surface $X_{0}$ and $\mathfrak{a} \subset \ell_{\infty}\left(\pi_{1}\left(X_{0}\right)\right)$ is a self-adjoint closed subalgebra.

The second Cousin problem for algebra $\mathcal{O}_{\mathfrak{a}}(X)$ asks about conditions for a divisor in $\operatorname{Div}_{\mathfrak{a}}(X)$ to be $\mathfrak{a}$-principal. Our next result provides some sufficient conditions for its solvability.

Theorem 2.20. Let $X_{0}$ be a Stein manifold and $E \in \operatorname{Div}_{\mathfrak{a}}(X)$. If $X_{0}$ is homotopy equivalent to an open subset $Y_{0} \subset X_{0}$ such that the restriction of $E$ to $Y:=p^{-1}\left(Y_{0}\right)$ is $\mathfrak{a}$-equivalent to an $\mathfrak{a}$-principal divisor, then $E$ is $\mathfrak{a}$-equivalent to an $\mathfrak{a}$-principal divisor as well.

In particular, the above conditions are satisfied if $\mathfrak{a}$ is such that $\hat{G}_{\mathfrak{a}}$ is a compact topological group and $j(G) \subset \hat{G}_{\mathfrak{a}}$ is a dense subgroup, and $\operatorname{supp}(E) \cap Y=\varnothing$; here $\operatorname{supp}(E)$ is the union of zero loci of holomorphic functions determining $E$.

For the algebra of Bohr's holomorphic almost periodic functions with $X$ and $Y$ being tube domains and $\mathfrak{a}=A P\left(\mathbb{Z}^{n}\right)$ (see Example 1.2) this theorem is due to [21] $(n=1)$ and [18] $(n \geq 1)$. The proof in [21] uses Arakelyan's theorem and gives an explicit construction of a holomorphic almost periodic function that determines the principal divisor. Similarly to [18] our proof of Theorem 2.20 is sheaf-theoretic.

The proofs of Theorems 2.17, 2.18, 2.20 and Proposition 2.19 are given in Section 10.

\subsection{Uniqueness sets for holomorphic a-functions}

A classical result by $\mathrm{H}$. Bohr states that if a holomorphic function $f$ on a complex strip $T:=\{z \in \mathbb{C}: \operatorname{Im}(z) \in(a, b)\}$, bounded on closed substrips, is continuous almost periodic on a horizontal line $\mathbb{R}+i c, c \in(a, b)$, then $f$ is holomorphic almost periodic on $T$. In this subsection we extend this result to algebras $\mathcal{O}_{\mathfrak{a}}(X)$.

The regular covering $p: X \rightarrow X_{0}$ is a principal fibre bundle over $X_{0}$ with structure group $G$, hence, for a cover $\left\{U_{0, \gamma}\right\}$ of $X_{0}$ by open simply connected subsets there exists a locally constant cocycle $\left\{c_{\delta \gamma}: U_{0, \gamma} \cap U_{0, \delta} \rightarrow G\right\}$ such that the covering $p: X \rightarrow X_{0}$ is obtained from the disjoint union $\sqcup_{\gamma} U_{0, \gamma} \times G$ by the identification

$$
U_{0, \delta} \times G \ni(x, g) \sim\left(x, g \cdot c_{\delta \gamma}(x)\right) \in U_{0, \gamma} \times G \quad \text { for all } x \in U_{0, \gamma} \cap U_{0, \delta},
$$

where projection $p$ is induced by the projections $U_{0, \gamma} \times G \rightarrow U_{0, \gamma}$ (see, e.g., [37]). Local inverses $\psi_{\gamma}: p^{-1}\left(U_{0, \gamma}\right) \rightarrow U_{0, \gamma} \times G$ to the identification map form a system of biholomorphic trivializations of the covering. For a given subset $S \subset G$ denote

$$
\Pi_{\gamma}\left(U_{0, \gamma}, S\right):=\psi_{\gamma}^{-1}\left(U_{0, \gamma} \times S\right)
$$


Now, let $U_{0} \subset X_{0}$ be an open simply connected set contained in some $U_{0, \gamma_{*}}$, $Z_{0} \subset U_{0}$ be a uniqueness set for holomorphic functions in $\mathcal{O}\left(X_{0}\right)$, and subsets $L \subset K \subset G$ be such that the closure of $j(L)$ in $\hat{G}_{\mathfrak{a}}$ is contained in the interior of the closure of $j(K)$ in $\hat{G}_{\mathfrak{a}}$ (see Subsection 2.1 for notation) and $\cup_{i=1}^{m} L \cdot g_{i}=G$ for some $g_{1}, \ldots, g_{m} \in G$.

Consider $Z \subset X$ such that

$$
p^{-1}\left(Z_{0}\right) \cap \Pi_{\gamma_{*}}\left(U_{0}, K\right) \subset Z .
$$

(In particular, we can take $L=K:=G$ and $Z:=p^{-1}\left(Z_{0}\right)$.)

We define $C_{\mathfrak{a}}(Z):=\left.C_{\mathfrak{a}}(X)\right|_{Z}$.

Theorem 2.21. If $f \in \mathcal{O}_{\ell_{\infty}(G)}(X)$ and $\left.f\right|_{Z} \in C_{\mathfrak{a}}(Z)$, then $f \in \mathcal{O}_{\mathfrak{a}}(X)$.

We prove Theorem 2.21 in Section 11.

Remark 2.22. (1) As an example of the uniqueness set $Z_{0}$ in Theorem 2.21 we can take any real hypersurface in $X_{0}$ or, more generally, a set of the form $\left\{x \in X_{0}: \rho_{1}(x)=\cdots=\rho_{d}(x)=0\right\}$, where $\rho_{1}, \ldots, \rho_{d}$ are real-valued differentiable functions on $X_{0}$ and $\partial \rho_{1}\left(x_{0}\right) \wedge \cdots \wedge \partial \rho_{d}\left(x_{0}\right) \neq 0$ for some $x_{0} \in Z_{0}$ (see, e.g., [3]).

(2) In the settings of the classical Bohr theorem the choice of the objects in Theorem 2.21 can be specified (recall that if $\mathfrak{a}=A P(G)$, then $\hat{G}_{\mathfrak{a}}$ is a compact topological group, cf. Example 3.3(2) in [15]):

Proposition 2.23. Suppose that $\mathfrak{a}$ is such that $\hat{G}_{\mathfrak{a}}$ is a compact topological group and $j(G) \subset \hat{G}_{\mathfrak{a}}$ is a dense subgroup. Given $K \subset G$ the following conditions are equivalent:

(a) There exist $g_{1}, \ldots, g_{m} \in G$ such that $\bigcup_{i=1}^{m} K \cdot g_{i}=G$;

(b) The closure of $j(K)$ in $\hat{G}_{\mathfrak{a}}$ has a nonempty interior;

(c) There exists a subset $L \subset K$ satisfying conditions of Theorem 2.21.

The proof of Proposition 2.23 is given in Section 11 .

Thus, for such algebras $\mathfrak{a}$ one can take as the set $K$ in Theorem 2.21, e.g., any nonempty subset of the form $\{g \in G:|f(g)|<1, f \in \mathfrak{a}\}$. For instance, in Bohr's result the line $\mathbb{R}+i c$ can be replaced with a set $S+K$, where $S \Subset T$ is an infinite set (hence, it is a uniqueness set for $\mathcal{O}(T))$ and $K:=\{n \in \mathbb{Z}:|E(n)|<1\} \neq \emptyset$, where $E$ is a univariate exponential polynomial of form (1.1).

\subsection{Leray, Hartogs and Peter-Weil-type theorems for algebras $\mathcal{O}_{\mathfrak{a}}(X)$}

In this subsection we do not assume that algebra $\mathfrak{a}$ is self-adjoint.

1. The following discussion suggests an alternative approach to study of $\mathcal{O}_{\mathfrak{a}}(X)$. Namely, we have an equivalent presentation of functions in $\mathcal{O}_{\mathfrak{a}}(X)$ as holomorphic sections of a holomorphic Banach vector bundle $\tilde{p}: C_{\mathfrak{a}} X_{0} \rightarrow X_{0}$ associated to the principal fibre bundle $p: X \rightarrow X_{0}$ and having fibre $\mathfrak{a}$ defined as follows. 
The regular covering $p: X \rightarrow X_{0}$ is a principal fibre bundle with structure group $G$ (see Subsection 2.4). Then $\tilde{p}: C_{\mathfrak{a}} X_{0} \rightarrow X_{0}$ is a holomorphic Banach vector bundle associated to $p: X \rightarrow X_{0}$ and having fibre $\mathfrak{a}$ obtained from the disjoint union $\sqcup_{\gamma} U_{0, \gamma} \times \mathfrak{a}$ by the identification

$$
U_{0, \delta} \times \mathfrak{a} \ni(x, f) \sim\left(x, R_{c_{\delta \gamma}(x)}(f)\right) \in U_{0, \gamma} \times \mathfrak{a} \quad \text { for all } x \in U_{0, \gamma} \cap U_{0, \delta} .
$$

The projection $\tilde{p}$ is induced by projections $U_{0, \gamma} \times \mathfrak{a} \rightarrow U_{0, \gamma}$.

Let $\mathcal{O}\left(C_{\mathfrak{a}} X_{0}\right)$ be the space of holomorphic sections of $C_{\mathfrak{a}} X_{0}$. This is a Fréchet algebra with respect to the usual pointwise operations and the topology of uniform convergence on compact subsets of $X_{0}$.

Proposition 2.24. $\mathcal{O}_{\mathfrak{a}}(X) \cong \mathcal{O}\left(C_{\mathfrak{a}} X_{0}\right)$.

We give proof of Proposition 2.24 in Section 12.

Using Proposition 2.24 we obtain the following result on extension within the class of holomorphic $\mathfrak{a}$-functions.

Proposition 2.25. Let $M_{0}$ be a closed complex submanifold of a Stein manifold $X_{0}, M:=p^{-1}\left(M_{0}\right) \subset X, D_{0} \Subset X_{0}$ is Levi strictly pseudoconvex (see, e.g., [34]), $D:=p^{-1}\left(D_{0}\right)$, and $f \in \mathcal{O}_{\mathfrak{a}}(M \cap D)$ is bounded. Then there exists a bounded function $F \in \mathcal{O}_{\mathfrak{a}}(D)$ such that $\left.F\right|_{M \cap D}=\left.f\right|_{M \cap D}$.

Indeed, subalgebra $\mathcal{O}_{\mathfrak{a}}(M)$ is isomorphic to the algebra $\left.\mathcal{O}\left(C_{\mathfrak{a}} X\right)\right|_{M_{0}}$ of holomorphic sections of bundle $C_{\mathfrak{a}} X$ over $M_{0}$. Since $X_{0}$ is Stein, there exist holomorphic Banach vector bundles $p_{1}: E_{1} \rightarrow X_{0}$ and $p_{2}: E_{2} \rightarrow X_{0}$ having complex Banach spaces $B_{1}$ and $B_{2}$ as their fibres, respectively, such that $E_{2}=E_{1} \oplus C_{\mathfrak{a}} X_{0}$ (the Whitney sum) and $E_{2}$ is holomorphically trivial, i.e., $E_{2} \cong X_{0} \times B_{2}$ (see, e.g., [55]). Thus, any holomorphic section of $E_{2}$ can be naturally identified with a $B_{2}$-valued holomorphic function on $X_{0}$. By $q: E_{2} \rightarrow C_{\mathfrak{a}} X_{0}$ and $i: C_{\mathfrak{a}} X_{0} \rightarrow E_{2}$ we denote the corresponding quotient and embedding homomorphisms of the bundles so that $q \circ i=\mathrm{Id}$. (Similar identifications hold for the bundle $C_{\mathfrak{a}} D_{0}$.) Given a function $\left.f \in \mathcal{O}\left(C_{\mathfrak{a}} X_{0}\right)\right|_{M_{0}}$ consider its image $\tilde{f}:=i(f)$, a $B_{2}$-valued holomorphic function on $M_{0}$, and apply to it the integral representation formula from [35] asserting the existence of a bounded function $\tilde{F} \in \mathcal{O}\left(D_{0}, B_{2}\right)$ such that $\left.\tilde{F}\right|_{M_{0} \cap D_{0}}=\left.\tilde{f}\right|_{M_{0} \cap D_{0}}$. Finally, we define $F:=q(\tilde{F})$.

In fact, this method allows to obtain similar extension results for holomorphic functions on $X$ whose restrictions to each fibre belong to some Banach space, and are possibly unbounded, see [8].

In view of Proposition 2.25, it is natural to ask to what extent Theorems 2.3, 2.4 and 2.10 depend on the assumption that the subalgebra $\mathfrak{a}$ is self-adjoint.

2. Next, we show that the classical Leray integral representation formula can be extended to work within subalgebra $\mathcal{O}_{\mathfrak{a}}(X)$.

For a given $z \in X_{0}$ by $\mathfrak{a}_{z}$ we denote the subalgebra of functions $h: p^{-1}(z) \rightarrow \mathbb{C}$ such that for all $x \in p^{-1}(z)$ functions $G \ni g \mapsto h(g \cdot x)$ are in $\mathfrak{a}$, endowed with sup-norm. Clearly, $\mathfrak{a}_{z}$ is isometrically isomorphic to $\mathfrak{a}$. 
Let

$$
D_{0} \Subset X_{0} \text { be a subdomain and } D:=p^{-1}\left(D_{0}\right) \text {. }
$$

We denote

$$
\mathcal{A}_{\mathfrak{a}}(D):=C_{\mathfrak{a}}(\bar{D}) \cap \mathcal{O}_{\mathfrak{a}}(D)
$$

(see Section 1 for definitions). This is a Banach space with respect to sup-norm.

Theorem 2.26. Let $X_{0}$ be a Stein manifold and $D_{0}, D$ be as above. There is a family of bounded linear operators $L_{z}: \mathfrak{a}_{z} \rightarrow \mathcal{A}_{\mathfrak{a}}(D), z \in D_{0}$, holomorphic in $z$ and such that

(1) $L_{z}(h)(x)=h(x)$ for all $h \in \mathfrak{a}_{z}, x \in p^{-1}(z)$;

(2) $\sup _{z \in D_{0}}\left\|L_{z}\right\|<\infty$.

We prove Theorem 2.26 in Section 12 .

Now, let us recall the classical Leray integral representation formula. For $\xi, \eta \in$ $\mathbb{C}^{n}$ we define $\langle\eta, \xi\rangle:=\sum_{k=1}^{n} \eta_{j} \xi_{j}$ and

$$
\begin{aligned}
\omega(\xi) & :=d \xi_{1} \wedge \cdots \wedge d \xi_{n} \\
\omega^{\prime}(\eta) & :=\sum_{k=1}^{n}(-1)^{k-1} \eta_{k} d \eta_{1} \wedge \cdots \wedge d \eta_{k-1} \wedge d \eta_{k+1} \wedge \cdots \wedge d \eta_{n} .
\end{aligned}
$$

For a domain $D_{0} \Subset \mathbb{C}^{n}$ we set $Q:=D_{0} \times \mathbb{C}^{n}$. Fix $z \in D_{0}$ and define a hypersurface $P_{z} \subset Q$ by

$$
P_{z}:=\{(\eta, \xi) \in Q:\langle\eta, \xi-z\rangle=0\} .
$$

Let $h_{z}$ be a $2 n-1$-dimensional cycle in $Q \backslash P_{z}$ whose projection to $D_{0}$ is homologous to $\partial D_{0}$.

Leray integral representation formula (see, e.g., [35]). For any function $f \in$ $\mathcal{O}\left(D_{0}\right)$

$$
f(z)=\frac{(n-1) !}{(2 \pi i)^{n}} \int_{h_{z}} f(\xi) \frac{\omega^{\prime}(\eta) \wedge \omega(\xi)}{\langle\eta, \xi-z\rangle^{n}} .
$$

Interpreting $z \mapsto L_{z}\left(\left.f\right|_{p^{-1}(z)}\right), z \in D_{0}, f \in \mathcal{O}_{\mathfrak{a}}(D)$, in Theorem 2.26 as an $\mathcal{A}_{\mathfrak{a}}(D)$-valued holomorphic function on $D_{0}$ and using the fact that representation (2.10) is valid for Banach-valued holomorphic functions (because the integral kernel in this formula is continuous and bounded on $h_{z}$ ) we obtain:

Theorem 2.27 (Leray-type integral representation formula). Let $X_{0} \subset \mathbb{C}^{n}$ be a Stein domain and $D_{0} \Subset X_{0}$ be a subdomain. Then for any function $f \in \mathcal{O}_{\mathfrak{a}}(D)$,

$$
f(x)=\frac{(n-1) !}{(2 \pi i)^{n}} \int_{h_{z}} L_{\xi}\left(\left.f\right|_{p^{-1}(\xi)}\right)(x) \frac{\omega^{\prime}(\eta) \wedge \omega(\xi)}{\langle\eta, \xi-z\rangle^{n}}, \quad \text { for all } x \in p^{-1}(z) .
$$

A similar formula for functions in $\mathcal{O}_{\ell_{\infty}}(D)$ was first established in [8]. 
3. Similarly to [7], we obtain the following Hartogs-type theorem.

Theorem 2.28. Suppose $n:=\operatorname{dim}_{\mathbb{C}} X_{0} \geq 2$. Let $D_{0} \Subset X_{0}$ be a subdomain with a connected piecewise $C^{1}$ boundary $\partial D_{0}$ contained in a Stein open submanifold of $X_{0}$ and $D:=p^{-1}\left(D_{0}\right)$. Assume that $f \in C_{\mathfrak{a}}(\partial D)$ satisfies the tangential CauchyRiemann equations on $\partial D$, i.e., for any smooth $(n, n-2)$-form $\omega$ on $X$ having compact support,

$$
\int_{\partial D} f \bar{\partial} \omega=0
$$

Then there exists a function $F \in \mathcal{O}_{\mathfrak{a}}(D) \cap C(\bar{D})$ such that $\left.F\right|_{\partial D}=f$.

The proof of Theorem 2.28 is given in Section 12 .

In particular, Theorem 2.28 implies that if $n \geq 2$, then each continuous almost periodic function on the boundary $\partial T=\mathbb{R}^{n}+i \partial \Omega$ of a tube domain $T:=\mathbb{R}^{n}+i \Omega \subset$ $\mathbb{C}^{n}$, where $\Omega \Subset \mathbb{R}^{n}$ is a domain with piecewise-smooth boundary $\partial \Omega$, satisfying the tangential Cauchy-Riemann equations on $\partial T$, admits a continuous extension to a holomorphic almost periodic function in $\mathcal{O}_{A P}(T) \cap C(\bar{T})$.

4. Now, we extend Bohr's approximation theorem for holomorphic almost periodic functions (see Section 1) to an arbitrary subalgebra $\mathcal{O}_{\mathfrak{a}}(X)$.

Let $\mathfrak{a}_{\iota}(\iota \in I)$ be a collection of closed subspaces of $\mathfrak{a}$ such that

(1) $\mathfrak{a}_{\iota}$ are invariant with respect to the action of $G$ on $\mathfrak{a}$ by right translates (i.e., if $f \in \mathfrak{a}_{\iota}$, then $R_{g}(f) \in \mathfrak{a}_{\iota}$ for all $\left.g \in G\right)$,

(2) the family $\left\{\mathfrak{a}_{\iota}: \iota \in I\right\}$ forms a direct system ordered by inclusion, and

(3) the linear space $\mathfrak{a}_{0}:=\bigcup_{\iota \in I} \mathfrak{a}_{\iota}$ is dense in $\mathfrak{a}$.

The model examples of subspaces $\mathfrak{a}_{\iota}$ are given in Subsection 3.5 below.

Let $\mathcal{O}_{\iota}(X)$ be the space of holomorphic functions $f \in \mathcal{O}_{\mathfrak{a}}(X)$ such that for every $x \in X$ functions

$$
g \mapsto f(g \cdot x), \quad g \in G,
$$

belong to $\mathfrak{a}_{\iota}$. Let $\mathcal{O}_{0}(X)$ be $\mathbb{C}$-linear hull of spaces $\mathcal{O}_{\iota}(X)$ with $\iota$ varying over $I$.

Theorem 2.29. If $X_{0}$ is a Stein manifold, then $\mathcal{O}_{0}(X)$ is dense in $\mathcal{O}_{\mathfrak{a}}(X)$.

We prove Theorem 2.29 in Section 12 .

If $\mathfrak{a}=A P(G)$ (see Subsections $3.1(2)$ and 3.2), then this theorem may be viewed as a holomorphic analogue of the Peter-Weyl approximation theorem.

\section{Examples}

\subsection{Examples of subalgebras $\mathfrak{a}$}

In addition to $\ell_{\infty}(G), c(G)$ and $A P\left(\mathbb{Z}^{n}\right)$ (cf. Section 1), we list the following examples of self-adjoint subalgebras of $\ell_{\infty}(G)$ separating points of $G$ and invariant with respect to the action of $G$ by right translations. 
(1) If group $G$ is residually finite (respectively, residually nilpotent), i.e., for any element $t \in G, t \neq e$, there exists a normal subgroup $G_{t} \not \ngtr t$ such that $G / G_{t}$ is finite (respectively, nilpotent), we consider the closed algebra $\hat{\ell}_{\infty}(G) \subset \ell_{\infty}(G)$ generated by pullbacks to $G$ of algebras $\ell_{\infty}\left(G / G_{t}\right)$ for all $G_{t}$ as above.

(2) Recall that a (continuous) bounded function $f$ on a (topological) group $G$ is called almost periodic if the families of its left and right translates

$$
\{t \mapsto f(s t)\}_{s \in G}, \quad\{t \mapsto f(t s)\}_{s \in G}
$$

are relatively compact in $\ell_{\infty}(G)$ (J. von Neumann [47]). (It was proved in [46] that the relative compactness of either the left or the right family of translates already gives the almost periodicity on $G$.) The algebra of almost periodic functions on $G$ is denoted by $A P(G)$.

The basic examples of almost periodic functions on $G$ are given by the matrix elements of the finite-dimensional irreducible unitary representations of $G$.

Recall that group $G$ is called maximally almost periodic if its finite-dimensional irreducible unitary representations separate points. Equivalently, $G$ is maximally almost periodic iff it admits a monomorphism into a compact topological group.

Any residually finite group belongs to this class. In particular, $\mathbb{Z}^{n}$, finite groups, free groups, finitely generated nilpotent groups, pure braid groups, fundamental groups of three dimensional manifolds are maximally almost periodic.

We denote by $A P_{0}(G) \subset A P(G)$ the space of functions

$$
t \mapsto \sum_{k=1}^{m} c_{k} \sigma_{i j}^{k}(t), \quad t \in G, \quad c_{k} \in \mathbb{C}, \quad \sigma^{k}=\left(\sigma_{i j}^{k}\right),
$$

where $\sigma^{k}(1 \leq k \leq m)$ are finite-dimensional irreducible unitary representations of $G$. The von Neumann approximation theorem [47] states that $A P_{0}(G)$ is dense in $A P(G)$.

In particular, the algebra $A P\left(\mathbb{Z}^{n}\right)$ of almost periodic functions on $\mathbb{Z}^{n}$ contains as a dense subset the subalgebra of exponential polynomials $t \mapsto \sum_{k=1}^{m} c_{k} e^{i\left\langle\lambda_{k}, t\right\rangle}$, $t \in \mathbb{Z}^{n}, \lambda_{k} \in \mathbb{R}^{n}, m \in \mathbb{N}$. Here $\left\langle\lambda_{k}, \cdot\right\rangle$ denotes the linear functional defined by $\lambda_{k}$.

(3) The algebra $A P_{\mathbb{Q}}\left(\mathbb{Z}^{n}\right)$ of almost periodic functions on $\mathbb{Z}^{n}$ with rational spectra. This is the subalgebra of $A P\left(\mathbb{Z}^{n}\right)$ generated over $\mathbb{C}$ by functions $t \mapsto e^{i\langle\lambda, t\rangle}$ with $\lambda \in \mathbb{Q}^{n}$.

(4) If group $G$ is finitely generated then, in addition to the subalgebra $c(G) \subset$ $\ell_{\infty}(G)$ of functions having limits at 'infinity', we can define a subalgebra $c_{E}(G) \subset$ $\ell_{\infty}(G)$ of functions having limits at 'infinity' along each 'path'.

To make this definition precise, we will need the notion of the end compactification of a connected and locally connected topological space $T$ that admits an exhaustion by compact subsets $K_{i}, i \in \mathbb{N}$, whose interiors cover $T$. Recall that the set of ends $E=E_{T}$ of space $T$ is the inverse limit of an inverse system of discrete spaces $\left\{\pi_{0}\left(T \backslash K_{i}\right)\right\}$, where $\pi_{0}\left(T \backslash K_{i}\right)$ is the set of connected components of $T \backslash K_{i}$, and each inclusion $T \backslash K_{j} \subset T \backslash K_{i}, i \leq j$, induces projection $\pi_{0}\left(T \backslash K_{j}\right) \rightarrow \pi_{0}\left(T \backslash K_{i}\right)$. The end compactification $\bar{T}_{E}$ of $T$ is a compact space 
defined as the disjoint union $T \sqcup E_{T}$ endowed with the weakest topology containing all open subsets of $T$ and all open neighbourhoods of the ends: an open neighbourhood of an end $e=\left\{e_{i} \in \pi_{0}\left(T \backslash K_{i}\right), i \in \mathbb{N}\right\}$ is a subset $V \subset T \sqcup E_{T}$ such that $V \cap E_{T}$ and $V \cap T$ are open in the corresponding topologies and $e_{i} \subset V \cap T$ for some $i \in \mathbb{N}$, see [23].

Now, suppose that group $G$ is finitely generated. By $\bar{G}_{E}$ we denote the end compactification of the Cayley graph $\mathcal{C}_{G}$ of $G$. Identifying naturally $G$ with the vertex set of $\mathcal{C}_{G}$ we define the subalgebra $c_{E}(G) \subset \ell_{\infty}(G)$ of functions admitting continuous extensions to $\bar{G}_{E}$. For example, if $G=\mathbb{Z}$, then $E=\{ \pm \infty\}$, and $c_{E}(\mathbb{Z})$ consists of functions $\mathbb{Z} \rightarrow \mathbb{C}$ having limits at $\pm \infty$.

(5) For a finitely generated group $G$, let $S A P(G) \subset \ell_{\infty}(G)$ denote the minimal subalgebra containing $A P(G)$ and $c_{E}(G)$. Elements of $S A P(G)$ are called semi-almost periodic functions (this is a variant of definition in $[49]$ for $G=\mathbb{R}$ ), see Example 3.3 below.

(6) Let $N$ be an infinite subgroup of $G$ and $N \backslash G$ be the set of (right) conjugacy classes. For a given class $N x \in N \backslash G$ endowed with the discrete topology by $c(N x)$ we denote the subalgebra of bounded functions $N x \rightarrow \mathbb{C}$ that admit extensions to the one-point compactification of $N x$. Let $c_{N}(G) \subset \ell_{\infty}(G)$ denote the subalgebra consisting of functions $h$ such that

$$
\left.h\right|_{N x} \in c(N x) \quad \text { for each } N x \in N \backslash G \text {. }
$$

(Thus, $h$ has limits 'at infinity' along each conjugacy class.)

Every function $h \in c_{N}(G)$ can be viewed as a bounded function on $N \backslash G$ with values in Banach algebra $c(N)$, i.e.,

$$
h \in \ell_{\infty}(N \backslash G, c(N)) .
$$

Instead of $\ell_{\infty}(N \backslash G, c(N))$ we may consider other Banach algebras of $c(N)$-valued functions on $N \backslash G$, e.g., $c(N \backslash G, c(N))$, thus obtaining other subalgebras of $\ell_{\infty}(G)$ satisfying assumptions of Section 1.

\subsection{Holomorphic almost periodic functions on coverings of complex manifolds}

In [15] we defined holomorphic almost periodic functions on a regular covering $X \rightarrow X_{0}$ as elements of algebra $\mathcal{O}_{A P}(X)$ (see Subsection 3.1(2) for the definition of algebra $A P=A P(G)$ ). Equivalently, a function $f \in \mathcal{O}(X)$ is called holomorphic almost periodic if each $G$-orbit in $X$ has a neighbourhood $U$ that is invariant with respect to the (left) action of $G$, such that the family of translates $\{z \mapsto f(g \cdot z), z \in U\}_{g \in G}$ is relatively compact in the topology of uniform convergence on $U$ (see [13] for the proof of the equivalence).

This is a variant of definition in [54], where $G$ is taken to be the group of all biholomorphic automorphisms of a complex manifold $X$ (see also [53]).

For instance, if $X_{0}$ is a non-compact Riemann surface and $p: X \rightarrow X_{0}$ is a regular covering with a maximally almost periodic deck transformation group $G$, 
then functions in $\mathcal{O}_{A P}(X)$ arise, e.g., as linear combinations over $\mathbb{C}$ of matrix entries of fundamental solutions of certain linear differential equations on $X$ (see Subsection 4.4 (2) for details).

We say that the covering $p: X \rightarrow X_{0}$ has the $\mathcal{O}_{\mathfrak{a}}$-Liouville property if $\mathcal{O}_{\mathfrak{a}}(X)$ does not contain non-constant bounded functions.

Recall that a complex manifold $X_{0}$ is called ultraliouville if there are no nonconstant bounded continuous plurisubharmonic functions on $X_{0}$ (e.g., connected compact complex manifolds and their Zariski open subsets are ultraliouville).

According to [44], if $X_{0}$ is ultraliouville and $G$ is virtually nilpotent (i.e., contains a nilpotent subgroup of finite index), then $X$ has the $\mathcal{O}_{\ell_{\infty}}$-Liouville property. For holomorphic almost periodic functions on $X$ this result can be strengthened, see Theorem 2.3 in [13]:

Let $p: X \rightarrow X_{0}$ be a regular covering of an ultraliouville complex manifold $X_{0}$. Then:

(1) $X$ has the $\mathcal{O}_{A P}$-Liouville property.

(2) Let $n \geq 2, D_{0} \Subset X_{0}$ be a subdomain with a connected piecewise smooth boundary $\partial D_{0}$ contained in a Stein open submanifold of $X_{0}$, and $D:=p^{-1}\left(D_{0}\right)$. Then $X \backslash D$ has $\mathcal{O}_{A P}$-Liouville property.

For instance, consider the universal covering $p: \mathbb{D} \rightarrow \mathbb{C} \backslash\{0,1\}$ of doubly punctured complex plane (here the deck transformation group is free group with two generators). Although there are plenty of non-constant bounded holomorphic functions on $\mathbb{D}$, all bounded holomorphic almost periodic functions on $\mathbb{D}$ corresponding to this covering are constant because $\mathbb{C} \backslash\{0,1\}$ is ultraliouville.

For other properties of algebra $\mathcal{O}_{A P}(X)$ see Subsection 4.3 below.

\subsection{Holomorphic semi-almost periodic functions}

Suppose that group $G$ is finitely generated. Elements of algebra $\mathcal{O}_{S A P}(X)$ (see Example $3.1(5)$ ) are called holomorphic semi-almost periodic functions. By Theorem 2.29 , the algebra $\mathcal{O}_{S A P}(X)$ is generated by subalgebras $\mathcal{O}_{A P}(X)$ (see Example 3.2) and $\mathcal{O}_{c_{E}}(X)$ (see Example $3.1(4)$ ). In the case $T \rightarrow T_{0}$ is a complex strip covering an annulus $T_{0}$ (see Example 1.2), algebra $\mathcal{O}_{S A P}(T)$ is related to the subalgebra of Hardy algebra $H^{\infty}(\mathbb{D})$ of bounded holomorphic functions on the unit disk $\mathbb{D} \subset \mathbb{C}$ generated by functions whose moduli have only the first-kind boundary discontinuities (see [14]).

\subsection{Examples of complex a-submanifolds}

We assume that the subalgebra $\mathfrak{a}$ is self-adjoint.

(1) If $Z_{0} \subset X_{0}$ is a complex submanifold of codimension $k$, then $Z:=p^{-1}\left(Z_{0}\right) \subset$ $X$ is a complex $\mathfrak{a}$-submanifold of codimension $k$.

(2) The disjoint union of a finite collection of complex a-submanifolds $Z_{i}$ of $X$ separated by functions in $C_{\mathfrak{a}}(X)$ (i.e., for each $i$ there is $f \in C_{\mathfrak{a}}(X)$ such that $f=1$ on $Z_{i}$ and $f=0$ on $Z_{j}$ for $j \neq i$ ) is a complex a-submanifold. 
(3) Let $Z_{0}=\left\{x \in X_{0}: f_{1}(x)=\cdots=f_{k}(x)=0\right\}$ for some $f_{i} \in \mathcal{O}\left(X_{0}\right)$ $(1 \leq i \leq k)$ be a complex submanifold of $X_{0}$ of codimension $k$. Set $Z:=p^{-1}\left(Z_{0}\right)$. Further, for an open subset $X_{0}^{\prime} \Subset X_{0}$ and functions $h_{1}, \ldots, h_{k} \in \mathcal{O}_{\mathfrak{a}}(X)$ we define $X^{\prime}:=p^{-1}\left(X_{0}^{\prime}\right), \delta:=\sup _{x \in X^{\prime}} \max _{1 \leq i \leq k}\left|h_{i}(x)\right|$, and

$$
Z_{h}:=\left\{x \in X: p^{*} f_{1}(x)+h_{1}(x)=\cdots=p^{*} f_{k}(x)+h_{k}(x)=0\right\} .
$$

Using the inverse function theorem with continuous dependence on parameter (Theorem 6.2), it is not difficult to see that $Z_{h}$ is a complex a-submanifold of $X^{\prime}$ provided that $\delta>0$ is sufficiently small.

(4) A complex a-submanifold of $X$ is called cylindrical if each open set $V$ in Definition 2.6 has form $V=p^{-1}\left(V_{0}\right)$ for some open $V_{0} \subset X_{0}$ (i.e., it is determined by holomorphic a-functions on preimages by $p$ of open subsets of $X_{0}$ ). If all complex $\mathfrak{a}$-submanifolds of $X$ were cylindrical, a much weaker version of Theorem 2.3 would have sufficed for the proof of the interpolation theorem for $\mathcal{O}_{\mathfrak{a}}(X)$ (Theorem 2.10). However, non-cylindrical a-submanifolds do exist: in [13] we constructed a noncylindrical $\mathfrak{a}$-hypersurface in $X$ in the case $\mathfrak{a}=A P(\mathbb{Z})$ (see Subsection 3.2) and $p: X \rightarrow X_{0}$ is a regular covering of a Riemann surface $X_{0}$ with deck transformation group $\mathbb{Z}$. We assumed that $X_{0}$ has finite type and is a relatively compact subdomain of a larger (non-compact) Riemann surface $\tilde{X}_{0}$ whose fundamental group satisfies $\pi_{1}\left(\tilde{X}_{0}\right) \cong \pi_{1}\left(X_{0}\right)$ (e.g., the covering of Example 1.2 with $n=1$, i.e., a complex strip covering an annulus, is a regular covering of this form).

Let us briefly describe this construction.

The covering $X$ of $X_{0}$ admits an injective holomorphic map into a holomorphic fibre bundle over $X_{0}$ having fibre $\left(\mathbb{C}^{*}\right)^{2}, \mathbb{C}^{*}:=\mathbb{C} \backslash\{0\}$, defined as follows. First, note that the regular covering $p: X \rightarrow X_{0}$ admits presentation as a principal fibre bundle with fibre $\mathbb{Z}$, see $(2.8)$. We choose two characters $\chi_{1}, \chi_{2}: \mathbb{Z} \rightarrow \mathbb{S}^{1} \cong \mathbb{R} /(2 \pi \mathbb{Z})$ such that the homomorphism $\left(\chi_{1}, \chi_{2}\right): \mathbb{Z} \rightarrow \mathbb{T}^{2}=\mathbb{S}^{1} \times \mathbb{S}^{1}$ is an embedding with dense image. Consider the fibre bundle $b_{\mathbb{T}^{2}} X$ over $X_{0}$ with fibre $\mathbb{T}^{2}$ associated with the principal fibre bundle $p: X \rightarrow X_{0}$ via the homomorphism $\left(\chi_{1}, \chi_{2}\right)$. The bundle $b_{\mathbb{T}^{2}} X$ is embedded into a holomorphic fibre bundle $b_{\left(\mathbb{C}^{*}\right)^{2}} X$ with fibre $\left(\mathbb{C}^{*}\right)^{2}$ associated with the composite of the embedding homomorphism $\mathbb{T}^{2} \hookrightarrow\left(\mathbb{C}^{*}\right)^{2}$ and $\left(\chi_{1}, \chi_{2}\right)$. Now, the covering $X$ of $X_{0}$ admits an injective $C^{\infty}$ map into $b_{\mathbb{T}^{2}} X$ with dense image and the composite of this map with the embedding of $b_{\mathbb{T}^{2}} X$ into $b_{\left(\mathbb{C}^{*}\right)^{2}} X$ is an injective holomorphic map $X \rightarrow b_{\left(\mathbb{C}^{*}\right)^{2}} X$. Further, the bundle $b_{\left(\mathbb{C}^{*}\right)^{2}} X$ admits a holomorphic trivialization $\eta: b_{\left(\mathbb{C}^{*}\right)^{2}} X \rightarrow X_{0} \times\left(\mathbb{C}^{*}\right)^{2}$. We choose $\chi_{1}(1)$ and $\chi_{2}(1)$ so close to $1 \in \mathbb{S}^{1}$ that the image $\eta\left(b_{\mathbb{T}^{2}} X\right) \subset X_{0} \times\left(\mathbb{C}^{*}\right)^{2}$ is sufficiently close to $X_{0} \times \mathbb{T}^{2}$. Thus identifying $X$ (by means of holomorphic injection $\left.X \hookrightarrow b_{\left(\mathbb{C}^{*}\right)^{2}} X \stackrel{\eta}{\rightarrow} X_{0} \times\left(\mathbb{C}^{*}\right)^{2}\right)$ with a subset of $X_{0} \times\left(\mathbb{C}^{*}\right)^{2}$, we obtain that $X$ is sufficiently close to $X_{0} \times \mathbb{T}^{2}$. Next, we construct a smooth complex hypersurface in $X_{0} \times\left(\mathbb{C}^{*}\right)^{2}$ such that in each cylindrical coordinate chart $U_{0} \times\left(\mathbb{C}^{*}\right)^{2}$ on $X_{0} \times\left(\mathbb{C}^{*}\right)^{2}$ for $U_{0} \Subset X_{0}$ simply connected it cannot be determined as the set of zeros of a holomorphic function on $U_{0} \times\left(\mathbb{C}^{*}\right)^{2}$. Intersecting this hypersurface with $X$ we obtain a non-cylindrical almost periodic hypersurface in $X$. (To construct such a hypersurface in $X_{0} \times\left(\mathbb{C}^{*}\right)^{2}$, we determine a smooth divisor in $\left(\mathbb{C}^{*}\right)^{2}$ that has a nonzero Chern class -i.e., it cannot be given by a holomorphic function on $\left.\left(\mathbb{C}^{*}\right)^{2}\right)$-, 
and whose support intersects the real torus $\mathbb{T}^{2} \subset\left(\mathbb{C}^{*}\right)^{2}$ transversely. Then we take the pullback of this divisor with respect to the projection $X_{0} \times\left(\mathbb{C}^{*}\right)^{2} \rightarrow\left(\mathbb{C}^{*}\right)^{2}$ to get the desired hypersurface.)

\subsection{Examples of spaces $\mathfrak{a}_{\iota}$ in Theorem 2.29}

(1) Let $\mathfrak{a}=\ell_{\infty}(G), I$ be the collection of all subsets of $G$ ordered by inclusion. It is easy to verify that given $\iota \in I$ we can define $\mathfrak{a}_{\iota}$ to be the closed linear subspace spanned by translates $\left\{R_{g}\left(\chi_{\iota}\right): g \in G\right\}$ of the characteristic function $\chi_{\iota}$ of subset $\iota$.

(2) Let $\mathfrak{a}=A P\left(\mathbb{Z}^{n}\right)$ (see Subsection $3.1(2)$ ). We can take $I$ to be the collection of all finite subsets of $\mathbb{R}^{n}$ ordered by inclusion and $\mathfrak{a}_{\iota}\left(\mathbb{Z}^{n}\right):=\operatorname{span}_{\mathbb{C}}\left\{t \mapsto e^{i\langle\lambda, t\rangle}, \lambda \in\right.$ $\left.\iota, \iota \in I, t \in \mathbb{Z}^{n}\right\}$.

We can also consider $\mathfrak{a}=A P_{\mathbb{Q}}\left(\mathbb{Z}^{n}\right)$, the algebra of almost periodic functions on $\mathbb{Z}^{n}$ having rational spectra (see Subsection $3.1(3)$ ). Here we take $I$ to be the collection of all finite subsets of $\mathbb{Q}^{n}$ ordered by inclusion and define spaces $\mathfrak{a}_{\iota}\left(\mathbb{Z}^{n}\right)$ similarly to the above.

(3) Let $\mathfrak{a}=A P(G)$ (see Subsection 3.1(2)) and $I$ consist of finite collections of finite-dimensional irreducible unitary representations of group $G$. We define $\mathfrak{a}_{\iota}(G)$, where $\iota=\left\{\sigma_{1}, \ldots, \sigma_{m}\right\} \in I$, to be the linear $\mathbb{C}$-hull of matrix elements $\sigma_{k}^{i j} \in A P(G)$ of representations $\sigma_{k}=\left(\sigma_{k}^{i j}\right), 1 \leq k \leq m$.

\section{Comments}

\subsection{Equivalent definition of holomorphic a-functions}

Let $\Lambda_{c}^{t, s}(X)$ denote the space of smooth $(t, s)$-forms on $X$ with compact supports endowed with the standard topology (see, e.g., [17]). Recall that continuous linear functionals on $\Lambda_{c}^{t, s}(X)$ are called $(n-t, n-s)$-currents.

There is an equivalent definition of holomorphic $\mathfrak{a}$-functions on a complex $\mathfrak{a}$ submanifold $Z$ (see Definition 2.8) in terms of currents. Namely, let $\mathfrak{a}$ be selfadjoint, then a function $f \in \mathcal{O}(Z)$ on a complex a-submanifold $Z \subset X$ is a holomorphic a-function if and only if it is bounded on subsets $Z \cap p^{-1}\left(U_{0}\right), U_{0} \Subset X_{0}$, and the corresponding current $c_{f}$,

$$
\left(c_{f}, \varphi\right):=\int_{Z} f \varphi, \quad \varphi \in \Lambda_{c}^{m, m}(X), \quad m:=\operatorname{dim}_{\mathbb{C}} Z,
$$

is an $\mathfrak{a}$-current meaning that for each $\varphi$ the function $G \ni g \mapsto\left(c_{f}, \varphi_{g}\right)$ belongs to algebra $\mathfrak{a}$; here $\varphi_{g}(x):=\varphi(g \cdot x)(x \in X)$. (The proof follows an argument in Proposition 2.4 of [19].)

In the setting of Example 1.2 (holomorphic almost periodic functions on tube domains) almost periodic currents were studied, e.g., in [22] (see further references therein). 


\subsection{Cylindrical a-divisors}

The class of $\mathfrak{a}$-principal divisors is contained in a larger class of cylindrical $\mathfrak{a}$ divisors, i.e., a-divisors determined by functions $f_{\alpha} \in \mathcal{O}_{\mathfrak{a}}\left(U_{\alpha}\right)$ with $U_{\alpha}=p^{-1}\left(U_{0, \alpha}\right)$ for some open $U_{0, \alpha} \subset X_{0}$ (see Definition 2.14).

If covering dimension of the maximal ideal space $M_{\mathfrak{a}}$ of $\mathfrak{a}$ is zero, then every $\mathfrak{a}$-divisor is $\mathfrak{a}$-equivalent to a cylindrical $\mathfrak{a}$-divisor (the latter follows from an equivalent definition of $\mathfrak{a}$-divisors as divisors on fibrewise compactification $c_{\mathfrak{a}} X$, see [13]). In particular, all $\ell_{\infty^{-}}, \hat{\ell}_{\infty}(G)$ - (for a residually finite group $\left.G\right), A P_{\mathbb{Q}^{-}}$ divisors (see (1) and (3) in Subsection 3.1) are $\ell_{\infty^{-}}, \hat{\ell}_{\infty}(G)-A P_{\mathbb{Q}^{-}}$equivalent to cylindrical divisors (see Examples $3.3(3)$ and $3.3(4)$ in [15]). There are, however, non-cylindrical $A P$-divisors, see Subsection 4.4 in [13].

For an $\mathfrak{a}$-divisor $E$ on $X$, Theorem 2.20 implies the following:

(a) If there exists a function $f \in \mathcal{O}_{\mathfrak{a}}(U)$, where $U=p^{-1}\left(U_{0}\right), U_{0} \subset X_{0}$ is open, such that $\left.E\right|_{U}$ is determined by $f$, then $E$ is a-equivalent to a cylindrical divisor (see the argument in the proof of Theorem 2.20).

(b) If $\mathfrak{a}=A P(G)$ and $E$ is not $\mathfrak{a}$-equivalent to a cylindrical $\mathfrak{a}$-divisor, then the projection of $\operatorname{supp}(E)$ to $X_{0}$ is dense (the converse is not true, see Subsection $3.4(4))$.

\subsection{Almost periodic divisors}

We use notation introduced in Subsection 4.1. Let $T_{E}$ be the current of integration of a divisor $E \in \operatorname{Div}(X)$, i.e.,

$$
\left(T_{E}, \varphi\right):=\int_{E} \varphi, \quad \varphi \in \Lambda_{c}^{n-1, n-1}(X)
$$

(see, e.g., [17]). One can prove that if $E \in \operatorname{Div}_{A P}(X)$, then current $T_{E}$ is almost periodic. Conversely, if the current of integration $T_{E}$ of a divisor $E \in \operatorname{Div}(X)$ is almost periodic, then $E$ is equivalent to an $A P$-divisor.

\subsection{Approximation of holomorphic almost periodic functions}

(1) Let $\mathcal{O}_{0}(T) \subset \mathcal{O}_{A P\left(\mathbb{Z}^{n}\right)}(T)$ be a subspace determined by the choice of spaces $\mathfrak{a}_{\iota}=\mathfrak{a}_{\iota}\left(\mathbb{Z}^{n}\right)(\iota \in I)$ as in Subsection $3.5(2)$. We show that exponential polynomials, see (1.1), are dense in $\mathcal{O}_{0}(T)$.

We denote $e_{\lambda}(t):=e^{i\langle\lambda, t\rangle}\left(\lambda \in \mathbb{R}^{n}, t \in \mathbb{Z}^{n}\right)$. Clearly, $e_{\lambda} \in \mathcal{O}_{\{\lambda\}}(T)$. Now, let $\iota=\left\{\lambda_{1}, \ldots, \lambda_{m}\right\}$. Since functions $e_{\lambda_{k}}(1 \leq k \leq m)$ are linearly independent in $\mathfrak{a}_{\iota}$, there exist linear projections $p_{\iota, \lambda_{k}}: \mathfrak{a}_{\iota} \rightarrow \mathfrak{a}_{\left\{\lambda_{k}\right\}}$. Since projections $p_{\iota, \lambda_{k}}$, $1 \leq k \leq m$, are invariant with respect to the action of $G$ on itself by right translates, they determine projections $P_{\iota, \lambda_{k}}: \mathcal{O}_{\iota}(T) \rightarrow \mathcal{O}_{\left\{\lambda_{k}\right\}}(T)$. (The latter follows, e.g., from the presentation of functions in $\mathcal{O}_{A P}(T)$ as sections of holomorphic Banach vector bundle $C_{A P} X_{0}$, see (2.9), where projections $P_{\iota, \lambda_{k}}$ become bundle homomorphisms $C_{\mathfrak{a}_{\iota}} X_{0} \rightarrow C_{\mathfrak{a}_{\left\{\lambda_{k}\right\}}} X_{0}$.) Therefore, there exist functions $f_{\lambda_{k}} \in \mathcal{O}_{\left\{\lambda_{k}\right\}}(T)$, $f_{\lambda_{k}}:=P_{\iota, \lambda_{k}}(f), 1 \leq k \leq m$, such that $f(z)=\sum_{k=1}^{m} f_{\lambda_{k}}(z), z \in T$. It is easy to see 
that for each $f_{\lambda_{k}}$ there exists a function $h_{\lambda_{k}} \in \mathcal{O}\left(T_{0}\right)$ such that $f_{\lambda_{k}} / e_{\lambda_{k}}=p^{*} h_{\lambda_{k}}$; hence,

$$
f(z)=\sum_{k=1}^{m}\left(p^{*} h_{\lambda_{k}}\right)(z) e^{i\left\langle\lambda_{k}, z\right\rangle}, \quad z \in T .
$$

Since the base $T_{0}$ of the covering is a relatively compact Reinhardt domain, functions $h_{\lambda_{k}}$ admit expansions into Laurent series (see, e.g., [50]):

$$
h_{k}(z)=\sum_{|\alpha|=-\infty}^{\infty} b_{\alpha} z^{\alpha}, \quad z \in T_{0}, \quad b_{t} \in \mathbb{C},
$$

where $\alpha=\left(\alpha_{1}, \ldots, \alpha_{n}\right)$ is a multiindex, $|\alpha|:=\alpha_{1}+\cdots+\alpha_{n}$. Since $p(z)=$ $\left(e^{i z_{1}}, \ldots, e^{i z_{n}}\right), z=\left(z_{1}, \ldots, z_{n}\right) \in T$ (see Example 1.2), each $p^{*} h_{\lambda_{k}}$ admits an approximation by finite sums

$$
\sum_{|\alpha|=-M}^{M} b_{\alpha} e^{i\langle\alpha, z\rangle}, \quad z \in T
$$

converging uniformly on subsets $p^{-1}\left(W_{0}\right) \subset T, W_{0} \Subset T_{0}$. Together with (4.2) this implies that exponential polynomials (1.1) are dense in $\mathcal{O}_{0}(T)$.

A similar argument shows that the algebra of holomorphic almost periodic functions with rational spectra (whose elements admit approximations by exponential polynomials (1.1) with $\lambda_{k} \in \mathbb{Q}^{n}$ ) coincides with algebra $\mathcal{O}_{A P_{\mathbb{Q}}}(T)$ (see Subsection $3.1(3))$.

(2) Let $X_{0}$ be a non-compact Riemann surface, $p: X \rightarrow X_{0}$ be a regular covering with a maximally almost periodic deck transformation group $G$ (for instance, $X_{0}$ is hyperbolic, $X=\mathbb{D}$ is its universal covering and $G=\pi_{1}\left(X_{0}\right)$ is a free (not necessarily finitely generated) group). Functions in $\mathcal{O}_{A P}(X)$ (see Subsection 3.2) arise, e.g., as linear combinations over $\mathbb{C}$ of matrix entries of fundamental solutions of certain linear differential equations on $X$.

Indeed, let $\mathcal{U}_{G}$ be the set of finite dimensional irreducible unitary representations $\sigma: G \rightarrow U_{m}(m \geq 1), I$ be the collection of finite subsets of $\mathcal{U}_{G}$ directed by inclusion, and for each $\iota \in I$ let $A P_{\iota}(G)$ be the (finite-dimensional) subspace generated by matrix elements of the unitary representations $\sigma \in \iota$. Then by Theorem 2.29 the $\mathbb{C}$-linear hull $\mathcal{O}_{0}(X)$ of spaces $\mathcal{O}_{\iota}(X)$ is dense in $\mathcal{O}_{\mathfrak{a}}(X)$ (note that for each $\sigma \in \mathcal{U}_{G}$ the space $\mathcal{O}_{\{\sigma\}}(X)$ is the $\mathbb{C}$-linear hull of coordinates of vectorvalued functions $f$ in $\mathcal{O}\left(X, \mathbb{C}^{m}\right)$ having the property that $f(g \cdot x)=\sigma(g) f(x)$ for all $g \in G, x \in X)$. Now, a unitary representation $\sigma: G \rightarrow U_{m}, m \geq 1$, can be obtained as the monodromy of the system $d F=\omega F$ on $X_{0}$, where $\omega$ is a holomorphic 1 -form on $X_{0}$ with values in the space of $m \times m$ complex matrices $M_{m}(\mathbb{C})$ (see, e.g., [24]). In particular, the system $d F=\left(p^{*} \omega\right) F$ on $X$ admits a global solution $F \in \mathcal{O}\left(X, G L_{m}(\mathbb{C})\right)$ such that $F \circ g^{-1}=F \sigma(g)(g \in G)$. By definition, a linear combination of matrix entries of $F$ is an element of $\mathcal{O}_{A P}(X)$. 


\subsection{Approximation property}

Recall that a (complex) Banach space $B$ is said to have the approximation property if for every compact set $K \subset B$ and every $\varepsilon>0$ there is a bounded operator $T=T_{\varepsilon, K} \in \mathcal{L}(B, B)$ of finite rank so that

$$
\|T x-x\|_{B}<\varepsilon \quad \text { for every } x \in K .
$$

For example, space $A P(G)$ of almost periodic functions on a group $G$ (see Subsection $3.1(2))$ has the approximation property with (approximation) operators $T$ in $\mathcal{L}\left(A P(G), A P_{0}(G)\right)$ (see, e.g., an argument in [51]).

In Subsection 2.5 suppose additionally to conditions (1)-(3) that

(4) the spaces $\mathfrak{a}_{\iota}, \iota \in I$, are finite-dimensional, and

(5) the space $\mathfrak{a}$ has the approximation property with approximation operators $S \in \mathcal{L}\left(\mathfrak{a}, \mathfrak{a}_{0}\right)$ equivariant with respect to the action of $G$ on $\mathfrak{a}$ by right translations, i.e., $S\left(R_{g}(f)\right)=R_{g}(S(f))$ for all $f \in \mathfrak{a}, g \in G$.

One can show that if $X_{0}$ is a Stein manifold and $D_{0} \Subset X_{0}$ is a strictly pseudoconvex domain, then the Banach space $\mathcal{A}_{\mathfrak{a}}(D):=\mathcal{O}_{\mathfrak{a}}(D) \cap C_{\mathfrak{a}}(\bar{D}), D:=p^{-1}\left(D_{0}\right)$, has the approximation property with approximation operators in $\mathcal{L}\left(\mathcal{A}_{\mathfrak{a}}(D), \mathcal{A}_{0}(D)\right)$ (here $\mathcal{A}_{0}(D)$ is defined similarly to $\mathcal{O}_{0}(D)$ in Theorem 2.29).

\section{Structure of fibrewise compactification $c_{\mathfrak{a}} X$}

In the present section we show that if algebra $\mathfrak{a}$ is self-adjoint, then there is an isomorphism between Fréchet algebras $\mathcal{O}\left(c_{\mathfrak{a}} X\right)$ (cf. Section 2) and $\mathcal{O}_{\mathfrak{a}}(X)$; therefore, complex function theories within $\mathcal{O}_{\mathfrak{a}}(X)$ and $\mathcal{O}\left(c_{\mathfrak{a}} X\right)$ are equivalent.

We refer to Sections 8 and 13 for the proofs of the results formulated in the present section.

\subsection{Complex structure}

A function $f \in C(U)$ on an open subset $U \subset c_{\mathfrak{a}} X$ is called holomorphic, i.e., belongs to the space $\mathcal{O}(U)$, if $\iota^{*} f$ is holomorphic on $V:=\iota^{-1}(U) \subset X$ in the usual sense (see Subsection 2.1 for notation).

Proposition 5.1. If $\mathfrak{a}$ is self-adjoint, then $C_{\mathfrak{a}}(V) \cong C(U)$ and $\mathcal{O}_{\mathfrak{a}}(V) \cong \mathcal{O}(U)$.

Let $U_{0} \subset X_{0}$ be open. A function $f \in C(U)$ on an open subset $U \subset U_{0} \times \hat{G}_{\mathfrak{a}}$ is called holomorphic if the function $\tilde{j}^{*} f$, where $\tilde{j}:=\operatorname{Id} \times j: U_{0} \times G \rightarrow U_{0} \times \hat{G}_{\mathfrak{a}}$, is holomorphic on the open subset $\tilde{j}^{-1}(U)$ of the complex manifold $U_{0} \times G$ (see Subsection 2.1 for the definition of the map $j$ ).

For sets $U$ as above, by $\mathcal{O}(U)$ we denote the algebra of holomorphic functions on $U$ endowed with the topology of uniform convergence on compact subsets of $U$. Clearly, $f \in C\left(c_{\mathfrak{a}} X\right)$ belongs to $\mathcal{O}\left(c_{\mathfrak{a}} X\right)$ if and only if each point in $c_{\mathfrak{a}} X$ has an open neighbourhood $U$ such that $\left.f\right|_{U} \in \mathcal{O}(U)$.

By $\mathcal{O}_{U}$ we denote the sheaf of germs of holomorphic functions on $U$. 
The category $\mathcal{M}$ of ringed spaces of the form $\left(U, \mathcal{O}_{U}\right)$, where $U$ is either an open subset of $c_{\mathfrak{a}} X$ and $X$ is a regular covering of a complex manifold $X_{0}$ or an open subset of $U_{0} \times \hat{G}_{\mathfrak{a}}$ with $U_{0} \subset X_{0}$ open, contains in particular complex manifolds.

Definition 5.2. A morphism of two objects in $\mathcal{M}$, that is, a map $F \in C\left(U_{1}, U_{2}\right)$, where $\left(U_{i}, \mathcal{O}_{U_{i}}\right) \in \mathcal{M}, i=1,2$, such that $F^{*} \mathcal{O}_{U_{2}} \subset \mathcal{O}_{U_{1}}$, is called a holomorphic map.

The collection of holomorphic maps $F: U_{1} \rightarrow U_{2},\left(U_{i}, \mathcal{O}_{U_{i}}\right) \in \mathcal{M}, i=1,2$, is denoted by $\mathcal{O}\left(U_{1}, U_{2}\right)$. If $F \in \mathcal{O}\left(U_{1}, U_{2}\right)$ has inverse $F^{-1} \in \mathcal{O}\left(U_{2}, U_{1}\right)$, then $F$ is called a biholomorphism.

Further, over each simply connected open subset $U_{0} \subset X_{0}$ there exists a biholomorphic trivialization $\psi=\psi_{U_{0}}: p^{-1}\left(U_{0}\right) \rightarrow U_{0} \times G$ of covering $p: X \rightarrow X_{0}$ which is a morphism of fibre bundles with fibre $G$ (see Subsection 2.4). Then there exists a biholomorphic trivialization $\bar{\psi}=\bar{\psi}_{U_{0}}: \bar{p}^{-1}\left(U_{0}\right) \rightarrow U_{0} \times \hat{G}_{\mathfrak{a}}$ of bundle $c_{\mathfrak{a}} X$ over $U_{0}$ which is a morphism of fibre bundles with fibre $\hat{G}_{\mathfrak{a}}$ such that the following diagram:

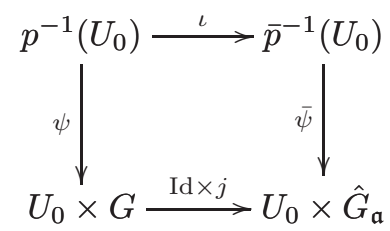

is commutative.

For a given subset $S \subset G$ we denote

$$
\Pi\left(U_{0}, S\right):=\psi^{-1}\left(U_{0} \times S\right)
$$

and identify $\Pi\left(U_{0}, S\right)$ with $U_{0} \times S$ where appropriate (here $\Pi\left(U_{0}, G\right)=p^{-1}\left(U_{0}\right)$ ).

For a subset $K \subset \hat{G}_{\mathfrak{a}}$ we denote

$$
\hat{\Pi}\left(U_{0}, K\right)\left(=\hat{\Pi}_{\mathfrak{a}}\left(U_{0}, K\right)\right):=\bar{\psi}^{-1}\left(U_{0} \times K\right) .
$$

A pair of the form $\left(\hat{\Pi}\left(U_{0}, K\right), \bar{\psi}\right)$ will be called a coordinate chart for $c_{\mathfrak{a}} X$. Similarly, sometimes we identify $\hat{\Pi}\left(U_{0}, K\right)$ with $U_{0} \times K$. If $K \subset \hat{G}_{\mathfrak{a}}$ is open, then, by our definitions, $\bar{\psi}^{*}: \mathcal{O}\left(U_{0} \times K\right) \rightarrow \mathcal{O}\left(\hat{\Pi}\left(U_{0}, K\right)\right)$ is an isomorphism of (topological) algebras.

\subsection{Basis of topology on $c_{\mathfrak{a}} X$}

By $\mathfrak{Q}$ we denote the basis of topology of $\hat{G}_{\mathfrak{a}}$ consisting of sets of the form

$$
\left\{\eta \in \hat{G}_{\mathfrak{a}}: \max _{1 \leq i \leq m}\left|h_{i}(\eta)-h_{i}\left(\eta_{0}\right)\right|<\varepsilon\right\}
$$

for all $\eta_{0} \in \hat{G}_{\mathfrak{a}}, h_{1}, \ldots, h_{m} \in C\left(\hat{G}_{\mathfrak{a}}\right)$, and $\varepsilon>0$.

The fibrewise compactification $c_{\mathfrak{a}} X$ is a paracompact Hausdorff space (as a fibre bundle with a paracompact base and a compact fibre); thus, $c_{\mathfrak{a}} X$ is a normal space. 
It is easy to see that the family

$$
\mathfrak{B}:=\left\{\hat{\Pi}\left(V_{0}, L\right) \subset c_{\mathfrak{a}} X: V_{0} \text { is open simply connected in } X_{0} \text { and } L \in \mathfrak{Q}\right\}
$$

forms a basis of topology of $c_{\mathfrak{a}} X$.

\subsection{Complex submanifolds}

To formulate the required definition note that for every $f \in \mathcal{O}\left(U_{0} \times K\right)$, where $U_{0} \subset X_{0}, K \subset \hat{G}_{\mathfrak{a}}$ are open, functions $f(\cdot, \omega), \omega \in K$, are in $\mathcal{O}\left(U_{0}\right)$. Indeed, since $f \in \mathcal{O}\left(U_{0} \times K\right)$, functions $U_{0} \ni z \mapsto f(z, j(g))\left(g \in j^{-1}(K)\right)$ are holomorphic. Then, since $j\left(j^{-1}(K)\right)$ is dense in $K$ (see Section 2) and $f$ is bounded on each $S \Subset U_{0} \times K$, by the Montel theorem $f(\cdot, \omega) \in \mathcal{O}\left(U_{0}\right)$ for all $\omega \in K$.

Definition 5.3. A closed subset $Y \subset c_{\mathfrak{a}} X$ is called a complex submanifold of codimension $k$ if for every $y \in Y$ there exist its neighbourhood of the form $U=$ $\hat{\Pi}\left(U_{0}, K\right) \subset c_{\mathfrak{a}} X$, where $U_{0} \subset X_{0}$ is open and simply connected, $K \subset \hat{G}_{\mathfrak{a}}$ is open, and functions $h_{1}, \ldots, h_{k} \in \mathcal{O}(U)$ such that

(1) $Y \cap U=\left\{x \in U: h_{1}(x)=\cdots=h_{k}(x)=0\right\}$;

(2) the rank of the map $z \mapsto\left(h_{1}(z, \omega), \ldots, h_{k}(z, \omega)\right)$ is $k$ at each point $x=$ $(z, \omega) \in Y \cap U$.

The next result describes the local structure of complex submanifolds of $c_{\mathfrak{a}} X$.

Proposition 5.4. Let $Y \subset c_{\mathfrak{a}} X$ be a complex submanifold. For every $y \in Y$ there exist an open neighbourhood $V \subset c_{\mathfrak{a}} X$ of $y$, open subsets $V_{0} \subset X_{0}$ and $K \subset \hat{G}_{\mathfrak{a}}$, a (closed) complex submanifold $Z_{0}$ of $V_{0}$ (of the same codimension as $Y$ ), and a biholomorphic map $\Phi \in \mathcal{O}\left(V_{0} \times K, V\right)$ such that $\Phi\left(V_{0} \times(K \cap j(G))\right)=V \cap \iota(X)$ and $\Phi^{-1}(V \cap Y)=Z_{0} \times K$.

The proof of Proposition 5.4, given in Subsection 6.3, is based on the inverse function theorem with continuous dependence on parameter (Theorem 6.2).

We use Proposition 5.4 to establish the following important fact (see Subsection 2.1 for the definition of a coherent sheaf on $\left.c_{\mathfrak{a}} X\right)$.

Proposition 5.5. The ideal sheaf $I_{Y}$ of germs of holomorphic functions vanishing on a complex submanifold $Y \subset c_{\mathfrak{a}} X$ is coherent.

Now, we list other properties of complex submanifolds of $c_{\mathfrak{a}} X$.

Proposition 5.6. Any complex submanifold $Y$ of $c_{\mathfrak{a}} X$ has the following properties:

(i) $\iota^{-1}(Y) \subset X$ is a complex submanifold of $X$ of codimension $k$.

(ii) $Y \cap \iota(X)$ is dense in $Y$.

Assertion (i) is immediate from the definition, while assertion (ii) follows from the fact that $\iota(X)$ is dense in $X$ combined with Proposition 5.4. 
Proposition 5.7. If $Z \subset X$ is a complex $\mathfrak{a}$-submanifold (see Definition 2.6), then the closure of $\iota(Z)$ in $c_{\mathfrak{a}} X$ is a complex submanifold of $c_{\mathfrak{a}} X$.

Suppose that $\mathfrak{a}$ is self-adjoint. If $Y$ is a complex submanifold of $c_{\mathfrak{a}} X$, then $\iota^{-1}(Y) \subset X$ is a complex $\mathfrak{a}$-submanifold.

Definition 5.8. A function $f \in C(Y)$ is called holomorphic if $\iota^{*} f \in \mathcal{O}\left(\iota^{-1}(Y)\right)$. The algebra of holomorphic functions on $Y$ is denoted by $\mathcal{O}(Y)$.

Similarly, we define holomorphic functions $\mathcal{O}(U)$ on an open subset $U \subset Y$ as those continuous functions whose pullbacks by $\iota$ are holomorphic in the usual sense.

Proposition 5.9. Suppose that $\mathfrak{a}$ is self-adjoint, $Y$ is a complex submanifold of $c_{\mathfrak{a}} X$. We set $Z:=\iota^{-1}(Y)$. Then $\mathcal{O}_{\mathfrak{a}}(Z) \cong \iota^{*} \mathcal{O}(Y)$ (so every function in $f \in \mathcal{O}_{\mathfrak{a}}(Z)$, see Definition 2.8, admits a unique extension to a function $\hat{f} \in \mathcal{O}(Y)$ such that $\left.f=\iota^{*} \hat{f}\right)$.

\subsection{Cartan theorems $\mathrm{A}$ and $\mathrm{B}$ on complex submanifolds}

The notion of a coherent sheaf on $c_{\mathfrak{a}} X$ (see Subsection 2.1) extends to analytic sheaves on a complex submanifold of $c_{\mathfrak{a}} X$. It turns out that if $X_{0}$ is a Stein manifold, then for such coherent sheaves we have analogues of Cartan theorems A and B (Theorems 5.11 and 5.12 below).

More precisely, we have the structure sheaf $\mathcal{O}_{Y}$ of germs of holomorphic functions on a complex submanifold $Y \subset c_{\mathfrak{a}} X$ (see Definition 5.8). A coherent sheaf $\mathcal{A}$ on $Y$ is a sheaf of modules over $\mathcal{O}_{Y}$ such that every point in $Y$ has a neighbourhood $V \subset Y$ over which, for every $N \geq 1$, there is a free resolution of $\mathcal{A}$ of length $N$, i.e., an exact sequence of sheaves of modules of the form

$$
\left.\left.\left.\left.\mathcal{O}_{Y}^{m_{N}}\right|_{V} \stackrel{\varphi_{N-1}}{\longrightarrow} \cdots \stackrel{\varphi_{2}}{\longrightarrow} \mathcal{O}_{Y}^{m_{2}}\right|_{V} \stackrel{\varphi_{1}}{\longrightarrow} \mathcal{O}_{Y}^{m_{1}}\right|_{V} \stackrel{\varphi_{0}}{\longrightarrow} \mathcal{A}\right|_{V} \longrightarrow 0
$$

(here $\varphi_{i}, 0 \leq i \leq N-1$, are homomorphisms of sheaves of modules).

Given a sheaf of modules $\mathcal{A}$ over $\mathcal{O}_{Y}$, we define a sheaf $\tilde{\mathcal{A}}$ on $c_{\mathfrak{a}} X$ (called the trivial extension of $\mathcal{A}$ ) by the formulas

$$
\left.\tilde{\mathcal{A}}\right|_{c_{\mathfrak{a}} X \backslash Y}:=0,\left.\quad \tilde{\mathcal{A}}\right|_{Y}:=\mathcal{A} .
$$

Using the results in [15], we establish the following.

Theorem 5.10. If $\mathcal{A}$ is a coherent sheaf on a complex submanifold $Y \subset c_{\mathfrak{a}} X$, then $\tilde{\mathcal{A}}$ is a coherent sheaf on $c_{\mathfrak{a}} X$.

It is immediate that $H^{k}(Y, \mathcal{A}) \cong H^{k}\left(c_{\mathfrak{a}} X, \tilde{\mathcal{A}}\right)$. Therefore, Theorems 2.3, 2.4 and Theorem 5.10 imply the following analogues of Cartan theorems A and B:

Let $\mathcal{A}$ be a coherent sheaf on a complex submanifold $Y \subset c_{\mathfrak{a}} X$ with $X_{0}$ Stein.

Theorem 5.11. Each stalk ${ }_{x} \mathcal{A}(x \in Y)$ is generated by global sections of $\mathcal{A}$ over $Y$ as an ${ }_{x} \mathcal{O}_{Y}$-module ( Cartan-type theorem $A$ ").

Theorem 5.12. Čech cohomology groups $H^{i}(Y, \mathcal{A})=0$ for all $i \geq 1$ ("Cartan-type theorem B"). 
Let $Y$ be either $c_{\mathfrak{a}} X$ or a complex submanifold of $c_{\mathfrak{a}} X$. The definition of coherence on $Y$ extends directly to open subsets of $Y$. It is natural to call such subset $W \subset Y$ a Stein manifold if all higher cohomology groups of coherent sheaves on $W$ vanish (i.e., Cartan-type theorem B holds on $W$ ). One can ask about characterization of Stein open submanifolds $W \subset Y$ (e.g., in terms of appropriately defined plurisubharmonic exhaustion $\mathfrak{a}$-functions on $W$ ).

\subsection{Dolbeault-type complex}

In this part we describe a Dolbeault-type complex and analogues of Dolbeault isomorphisms used in the proof of Proposition 2.19.

Let $Y \subset c_{\mathfrak{a}} X$ be a complex submanifold. We define the holomorphic tangent bundle $T Y$ of $Y$ as a holomorphic bundle on $Y$ whose pullback by $\iota$ to $\iota^{-1}(Y)$ coincides with the holomorphic tangent bundle of the complex submanifold $\iota^{-1}(Y) \subset X$ (see the proof of Theorem 2.9 in Section 9 for existence and uniqueness of $T Y$ ).

The definition of the antiholomorphic tangent bundle $\overline{T Y}$ of $Y$ is analogous.

We define the complexified tangent bundle of $Y$ as the Whitney sum

$$
T^{\mathbb{C}} Y:=T Y \oplus \overline{T Y}
$$

By $\Lambda_{c}^{m}(Y):=\Gamma\left(Y, \wedge^{m}\left(T^{\mathbb{C}} Y\right)^{*}\right)$ we denote the space of continuous sections of the vector bundle $\wedge^{m}\left(T^{\mathbb{C}} Y\right)^{*}\left(0 \leq m \leq n:=\operatorname{dim}_{\mathbb{C}} X_{0}\right)$, where $\left(T^{\mathbb{C}} Y\right)^{*}$ is the dual bundle of $T^{\mathbb{C}} Y$. Elements of $\Lambda_{c}^{m}(Y)$ will be called continuous $m$-forms.

By Proposition 5.4, for every point $x \in Y$ there exist a neighbourhood $U \subset c_{\mathfrak{a}} X$, a biholomorphism $\varphi: U \rightarrow U_{0} \times K$, where $U_{0} \subset \mathbb{C}^{n}, K \subset \hat{G}_{\mathfrak{a}}, K \in \mathfrak{Q}$ (see (5.3)) are open, and a complex submanifold $Y_{0} \subset U_{0}$ such that $\varphi(Y \cap U)=Y_{0} \times K$ and $\varphi(Y \cap U \cap \iota(X))=Y_{0} \times(K \cap j(G))$. By $\wedge^{m} T^{\mathbb{C}}\left(Y_{0} \times K\right)^{*}$ we denote the pullback to $Y_{0} \times K$ of the bundle $\wedge^{m}\left(T^{\mathbb{C}} Y_{0}\right)^{*}$ under the natural projection $Y_{0} \times K \rightarrow Y_{0}$. Since $\left.\varphi^{-1}\right|_{Y_{0} \times(K \cap j(G))}: Y_{0} \times(K \cap j(G)) \rightarrow Y \cap U \cap \iota(X)$ is a biholomorphism of usual complex manifolds,

$$
\left(\left.\varphi^{-1}\right|_{Y_{0} \times(K \cap j(G))}\right)^{*}\left(\wedge^{m}\left(T^{\mathbb{C}} Y\right)^{*}\right)=\left.\wedge^{m} T^{\mathbb{C}}\left(Y_{0} \times K\right)^{*}\right|_{Y_{0} \times(K \cap j(G))} .
$$

Since $Y_{0} \times(K \cap j(G))$ is dense in $Y_{0} \times K$, the latter bundle is dense in the bundle $\wedge^{m} T^{\mathbb{C}}\left(Y_{0} \times K\right)^{*}$. Thus the above identity and the continuity of $\varphi^{-1}$ imply that $\left(\varphi^{-1}\right)^{*}\left(\wedge^{m}\left(T^{\mathbb{C}} Y\right)^{*}\right)=\wedge^{m} T^{\mathbb{C}}\left(Y_{0} \times K\right)^{*}$. In particular, $\left(\varphi^{-1}\right)^{*} \operatorname{maps} \Lambda_{c}^{m}(Y \cap U)$ to $\Lambda_{c}^{m}\left(Y_{0} \times K\right)$, the space of continuous sections of $\wedge^{m} T^{\mathbb{C}}\left(Y_{0} \times K\right)^{*}$. Clearly,

$$
\Lambda_{c}^{m}\left(Y_{0} \times K\right)=C\left(Y_{0} \times K\right) \otimes \Lambda_{c}^{m}\left(Y_{0}\right),
$$

where $\Lambda_{c}^{m}\left(Y_{0}\right)$ is the space of continuous $m$-forms on $Y_{0}$ and $C\left(Y_{0} \times K\right)$ is the space of continuous functions on $Y_{0} \times K$ endowed with the Fréchet topology of uniform convergence on compact subsets of $Y_{0} \times K$.

By $\Lambda^{m}(Y) \subset \Lambda_{c}^{m}(Y)$ we denote the subspace of $C^{\infty} m$-forms, that is, forms $\omega$ such that for each "coordinate map" $\varphi: U \rightarrow U_{0} \times K$,

$$
\left.\left(\varphi^{-1}\right)^{*} \omega\right|_{Y \cap U} \in \Lambda^{m}\left(Y_{0} \times K\right):=C^{\infty}\left(Y_{0} \times K\right) \otimes \Lambda^{m}\left(Y_{0}\right),
$$


where $\Lambda^{m}\left(Y_{0}\right)$ is the space of $C^{\infty} m$-forms on $Y_{0}$ and $C^{\infty}\left(Y_{0} \times K\right) \subset C\left(Y_{0} \times K\right)$ is the subspace of continuous functions that are $C^{\infty}$ when viewed as functions on $Y_{0}$ taking values in the Fréchet space $C(K)$.

We denote $C^{\infty}(Y):=\Lambda^{0}(Y)$.

Lemma 5.13. $\Lambda^{m}(Y)$ is correctly defined by (local) conditions (5.7).

Let $a \in C^{\infty}\left(Y_{0} \times K\right)$. We define the differential $d a \in \Lambda^{1}\left(Y_{0} \times K\right)$ as follows. (To simplify notation, we may assume without loss of generality that $Y_{0}$ is an open subset of $\mathbb{C}^{n-k}$.)

Define $d a:=\sum_{i=1}^{n-k} \frac{\partial a}{\partial z_{i}} d z_{i}$, where $\partial a / \partial z_{i} \in C^{\infty}\left(Y_{0} \times K\right)$ is the derivative of the Fréchet-valued map $z \mapsto a(z, \cdot) \in C(K), z=\left(z_{1}, \ldots, z_{n-k}\right) \in Y_{0}$, with respect to $z_{i}$.

We have the operator of differentiation $d: \Lambda^{m}\left(Y_{0} \times K\right) \rightarrow \Lambda^{m+1}\left(Y_{0} \times K\right)$ defined by the formula

$$
d\left(\sum_{i=1}^{l} a_{i} \omega_{i}\right):=\sum_{i=1}^{l} a_{i} d \omega_{i}+\sum_{i=1}^{l} d a_{i} \wedge \omega_{i}, \quad a_{i} \in C^{\infty}\left(Y_{0} \times K\right), \quad \omega_{i} \in \Lambda^{m}\left(Y_{0}\right) .
$$

Now, we define the operator of differentiation $d: \Lambda^{m}(Y) \rightarrow \Lambda^{m+1}(Y)$ :

For each coordinate map $\varphi: U \rightarrow U_{0} \times K$ and $\omega \in \Lambda^{m}(Y)$ the form $d \omega \in$ $\Lambda^{m+1}(Y)$ satisfies

$$
\left(\varphi^{-1}\right)^{*} d \omega=d\left(\left(\varphi^{-1}\right)^{*} \omega\right)
$$

where the right-hand side is defined by (5.8).

Existence of $d \omega$ satisfying local conditions (5.9) follows from the facts that due to these conditions $\left.\iota^{*} \circ d\right|_{\left.\Lambda^{m}(Y)\right|_{U}}=\left.d \circ \iota^{*}\right|_{\left.\Lambda^{m}(Y)\right|_{U}}$, where the $d$ on the right denotes the standard differentiation on the space of differential forms defined on the complex submanifold $\iota^{-1}(Y) \subset X$, and that $\iota\left(\iota^{-1}(Y)\right)$ is dense in $Y$ (see Proposition 5.4). By the same reason we have $d \circ d=0$.

Further, (5.5) induces decomposition $\left(T^{\mathbb{C}} Y\right)^{*}=T Y^{*} \oplus \overline{T Y}^{*}$ and, hence,

$$
\Lambda^{m}(Y)=\oplus_{p+k=m} \Lambda^{p, k}(Y),
$$

where

$$
\Lambda^{p, k}(Y):=\Gamma\left(Y, \wedge^{p} T Y^{*} \otimes \wedge^{q} \overline{T Y}^{*}\right) \cap \Lambda^{m}(Y) .
$$

Since the pullback by $\iota$ of $T Y^{*}$ coincides with the holomorphic cotangent bundle of complex submanifold $\iota^{-1}(Y) \subset X$, the pullback by $\iota$ of decomposition $(5.10)$ agrees with the usual type decomposition of differential forms on $\iota^{-1}(Y)$.

Using the natural projections $\pi^{p, k}: \Lambda^{m}(Y) \rightarrow \Lambda^{p, k}(Y)(m=p+k)$, we define

$$
\partial:=\pi^{p+1, k} \circ d, \quad \bar{\partial}:=\pi^{p, k+1} \circ d .
$$

Since pullbacks by $\iota$ of these operators coincide with their usual counterparts on the complex submanifold $\iota^{-1}(Y) \subset X$ and the image by $\iota$ of the latter is dense in $Y$, we have $\partial \circ \partial=0, \bar{\partial} \circ \bar{\partial}=0$ and $d=\partial+\bar{\partial}$.

The above definitions and notation transfer naturally to open subsets of $Y$. In particular, we can define the sheaf $\Lambda^{p, k}$ of germs of $C^{\infty}(p, q)$-forms on $Y$. 
Lemma 5.14. For any open cover $\mathcal{U}$ of $Y$ there is a subordinate $C^{\infty}$ partition of unity.

This lemma implies that $\Lambda^{p, k}$ is a fine sheaf, and therefore cohomology groups $H^{r}\left(Y, \Lambda^{p, k}\right)=0$ for all $r \geq 1$ (see, e.g., [34]).

Let $Z^{p, k} \subset \Lambda^{p, k}$ denote the subsheaf of germs of $\bar{\partial}$-closed forms. We have the following analogue of $\bar{\partial}$-Poincaré lemma for sections of $Z^{p, k}$.

Proposition 5.15. Let $Y \subset c_{\mathfrak{a}} X$ be a complex submanifold. For every point $x \in Y$ there are neighbourhoods $W, V \subset Y, W \Subset V$, of $x$ such that restriction to $W$ of any $\bar{\partial}$-closed form in $\Lambda^{p, k+1}(V)$ is $\bar{\partial}$-exact.

Let $Z^{p, k}(Y) \subset \Lambda^{p, k}(Y)$ denote the subspace of $\bar{\partial}$-closed forms. We define the Dolbeault cohomology groups of $Y$ as

$$
\begin{aligned}
& H^{p, k}(Y):=Z^{p, k}(Y) / \bar{\partial} \Lambda^{p, k-1}(Y), \quad p \geq 0, \quad k \geq 1, \\
& H^{p, 0}(Y):=Z^{p, 0}(Y) .
\end{aligned}
$$

We set $\Omega^{p}:=Z^{p, 0}$. Then $\Omega^{p}$ is the sheaf of germs of holomorphic $p$-forms on $Y$, i.e., holomorphic sections of the bundle $\wedge^{p} T Y^{*}$. (Note that $\iota^{*} \Omega^{p}$ is the sheaf of germs of usual holomorphic $p$-forms on the complex submanifold $\iota^{-1}(Y) \subset X$.)

Since $\Lambda^{p, k}$ is a fine sheaf, from Proposition 5.15 and a standard result in Chapter $\mathrm{B}, \S 1.3$ of [29], we obtain:

Corollary 5.16 (Dolbeault-type isomorphism). $\forall p, k \geq 0, H^{p, k}(Y) \cong H^{k}\left(Y, \Omega^{p}\right)$.

Since $\Omega^{p}$ is the sheaf of germs of sections of a holomorphic vector bundle on $Y$, it is coherent (see Subsection 5.4). Then the previous corollary and Theorem 5.12 imply

Corollary 5.17. Suppose that $X_{0}$ is a Stein manifold, $Y \subset c_{\mathfrak{a}} X$ is a complex submanifold. Then

$$
H^{p, k}(Y)=0 \quad \text { for all } \quad p \geq 0, \quad k \geq 1
$$

(i.e., any $\bar{\partial}$-closed form in $\Lambda^{p, k}(Y)$ is $\bar{\partial}$-exact).

Similarly one can define the de Rham cohomology groups of $Y$ and obtain an analogue of the classical de Rham isomorphism (see the proof of Proposition 2.19).

\subsection{Characterization of $c_{\mathfrak{a}} X$ as the maximal ideal space of $\mathcal{O}_{\mathfrak{a}}(X)$}

Now we relate the fibrewise compactification $c_{\mathfrak{a}} X$ of covering $p: X \rightarrow X_{0}$ to the maximal ideal space $M_{X}$ of algebra $\mathcal{O}_{\mathfrak{a}}(X)$, i.e., the space of non-zero characters $\mathcal{O}_{\mathfrak{a}}(X) \rightarrow \mathbb{C}$ endowed with weak ${ }^{*}$ topology $\left(\right.$ of $\left.\mathcal{O}_{\mathfrak{a}}(X)^{*}\right)$.

Theorem 5.18. Suppose that algebra $\mathfrak{a}$ is self-adjoint, and $X_{0}$ is a Stein manifold. Then $M_{X}$ is homeomorphic to $c_{\mathfrak{a}} X$. 
Since $\iota(X)$ is dense in $c_{\mathfrak{a}} X$, and the natural mapping of $X$ into $M_{X}$, sending each point of $X$ to its point evaluation homomorphism, coincides with $\iota$ under the homeomorphism of Theorem 5.18, we obtain the following corona-type theorem.

Corollary 5.19. Let $\mathfrak{a}$ be self-adjoint, $X_{0}$ be a Stein manifold. Then $\iota(X)$ is dense in $M_{X}$.

\section{Proofs: Preliminaries}

\section{1. Čech cohomology}

For a topological space $X$ and a sheaf of abelian groups $\mathcal{S}$ on $X$ by $\Gamma(X, \mathcal{S})$ we denote the abelian group of continuous sections of $\mathcal{S}$ over $X$.

Let $\mathcal{U}$ be an open cover of $X$. By $C^{i}(\mathcal{U}, \mathcal{S})$ we denote the space of Čech $i$ cochains with values in $\mathcal{S}$, by $\delta: C^{i}(\mathcal{U}, \mathcal{S}) \rightarrow C^{i+1}(\mathcal{U}, \mathcal{R})$ the Cech coboundary operator, by $Z^{i}(\mathcal{U}, \mathcal{S}):=\left\{\sigma \in C^{i}(\mathcal{U}, \mathcal{S}): \delta \sigma=0\right\}$ the space of $i$-cocycles, and by $B^{i}(\mathcal{U}, \mathcal{S}):=\left\{\sigma \in Z^{i}(\mathcal{U}, \mathcal{S}): \sigma=\delta(\eta), \eta \in \mathcal{C}^{i-1}(\mathcal{U}, \mathcal{S})\right\}$ the space of $i$-coboundaries (see, e.g., [34] for details). The Cech cohomology groups $H^{i}(\mathcal{U}, S), i \geq 0$, are defined by

$$
H^{i}(\mathcal{U}, S):=Z^{i}(\mathcal{U}, \mathcal{S}) / B^{i}(\mathcal{U}, \mathcal{S}), \quad i \geq 1,
$$

and $H^{0}(\mathcal{U}, \mathcal{S}):=\Gamma(\mathcal{U}, \mathcal{S})$

\section{2. $\bar{\partial}$-equation}

Let $B$ be a complex Banach space, $D_{0} \subset X_{0}$ be a strictly pseudoconvex domain. We fix a system of local coordinates on $D_{0}$. Let $\left\{W_{0, i}\right\}_{i \geq 1}$ be the cover of $D_{0}$ by the coordinate patches. By $\Lambda_{b}^{(0, q)}\left(D_{0}, B\right), q \geq 0$, we denote the space of bounded continuous $B$-valued $(0, q)$-forms on $D_{0}$ endowed with norm

$$
\|\omega\|_{D_{0}}=\|\omega\|_{D_{0}, B}^{(0, q)}:=\sup _{x \in W_{0, i}, i \geq 1, \alpha}\left\|\omega_{\alpha, i}(x)\right\|_{B},
$$

where $\omega_{\alpha, i}\left(\alpha\right.$ is a multiindex) are coefficients of forms $\left.\omega\right|_{W_{0, i}} \in \Lambda_{b}^{(0, q)}\left(W_{0, i}, B\right)$ in local coordinates on $W_{0, i}$.

The next lemma follows easily from results in [35] (proved for $B=\mathbb{C}$ ) because all integral representations and estimates are preserved when passing to the case of Banach-valued forms.

Lemma 6.1. There exists a bounded linear operator

$$
R_{D_{0}, B} \in \mathcal{L}\left(\Lambda_{b}^{(0, q)}\left(D_{0}, B\right), \Lambda_{b}^{(0, q-1)}\left(D_{0}, B\right)\right), \quad q \geq 1
$$

such that if $\omega \in \Lambda_{b}^{(0, q)}\left(D_{0}, B\right)$ is $C^{\infty}$ and $\bar{\partial}$-closed on $D_{0}$, then $\bar{\partial} R_{D_{0}, B}(\omega)=\omega$. 


\subsection{Inverse function theorem with continuous dependence on the pa- rameter}

Theorem 6.2. Let $K$ be a topological space, $B_{1}, B_{2} \Subset \mathbb{C}^{n}$ be open balls centered at the origin. Fix a point $\left(x_{0}, \eta_{0}\right) \in B_{1} \times K$. Suppose that a continuous map $G: B_{1} \times K \rightarrow B_{2}$ satisfies

(1) $G(\cdot, \eta): B_{1} \rightarrow B_{2}$ is holomorphic for every $\eta \in K$,

(2) the Jacobian matrix $D_{x} G\left(x_{0}, \eta_{0}\right)$ is non-degenerate.

Then there exist an open subset $W \subset B_{2} \times K$ and a continuous map $H: W \rightarrow B_{1}$ such that

(a) $\left(G\left(x_{0}, \eta_{0}\right), \eta_{0}\right) \in W$,

(b) $H(\cdot, \eta)$ is holomorphic on $W \cap\left(B_{2} \times\{\eta\}\right)$ for all $\eta$ for which this set is non-empty,

(c) $G(H(z, \eta), \eta)=z$ for all $(z, \eta) \in W$.

Theorem 6.2 follows easily from the contraction principle with continuous dependence on parameter, see e.g., Chapter XVI of [39].

As an application of Theorem 6.2 we prove Proposition 5.4 on the local structure of complex submanifolds of $c_{\mathfrak{a}} X$.

Proof of Proposition 5.4. Let $Y \subset c_{\mathfrak{a}} X$ be a complex submanifold and $y_{0} \in Y$. In notation of Definition 5.3, there exists a neighbourhood $U:=\hat{\Pi}\left(U_{0}, L\right) \subset c_{\mathfrak{a}} X$ of $y_{0}$, where $U_{0} \subset X_{0}$ is a simply connected coordinate chart and $L \subset \hat{G}_{\mathfrak{a}}$ is open, such that $Y \cap U=\left\{y \in U: h_{1}(y)=\cdots=h_{k}(y)=0\right\}$ with $h_{i} \in \mathcal{O}(U)(1 \leq i \leq k)$ satisfying non-degeneracy condition (2) of the definition.

Since sets $U$ and $U_{0} \times L$ are biholomorphic (see Subsection 5.1), in what follows we identify them. Next, since functions $h_{i}$ satisfy the non-degeneracy condition of Definition 5.3, we may choose coordinates $x_{1}, \ldots, x_{n}$ on $U_{0}$ so that the Jacobian matrix $D_{x} G\left(x_{0}, \eta_{0}\right), y:=\left(x_{0}, \eta_{0}\right)$, of the map

$G(x, \eta):=\left(h_{1}(x, \eta), \ldots, h_{k}(x, \eta), x_{k+1}, \ldots, x_{n}\right), x:=\left(x_{1}, \ldots, x_{n}\right),(x, \eta) \in U_{0} \times L$,

is non-degenerate. Also, we may assume without loss of generality that $U_{0}=B_{1}$ and $G\left(B_{1}, \eta\right) \subset B_{2}$ for all $\eta \in L$, where $B_{i} \Subset \mathbb{C}^{n}, i=1,2$, are open balls centered at the origin. Hence, we can apply Theorem 6.2. In its notation, shrinking $W$, if necessary, we may assume that $W=V_{0} \times K$ for some open $V_{0} \subset B_{2}, K \subset L$ which we take as the required sets in the formulation of Proposition 5.4. We also take $\Phi(z, \eta):=(H(z, \eta), \eta),(z, \eta) \in V_{0} \times K$, and $V:=\Phi\left(V_{0} \times K\right) \subset U$. By definition, $\Phi \in \mathcal{O}\left(V_{0} \times K, V\right)$ (see Subsection 5.1). Further, since $(G \circ \Phi(z), \eta)=(z, \eta)$ for all $(z, \eta) \in V_{0} \times K$

$$
\left(h_{i} \circ \Phi\right)\left(z_{1}, \ldots, z_{n}, \eta\right)=z_{i}, \quad(z, \eta) \in V_{0} \times K, \quad z=\left(z_{1}, \ldots, z_{n}\right), \quad 1 \leq i \leq k .
$$

Therefore, $\Phi^{-1}(V \cap Y)=Z_{0} \times K$, where $Z_{0}:=\left\{\left(0, \ldots, 0, z_{k+1}, \ldots, z_{n}\right) \in V_{0}:\right.$ $\left.\left(z_{1}, \ldots, z_{n}\right) \in V_{0}\right\}$ is a complex submanifold of codimension $k$.

By our construction we also have $\Phi\left(V_{0} \times(K \cap j(G))\right)=V \cap \iota(X)$.

The proof of the proposition is complete. 


\section{Proof of Theorem 1.3}

Fix some $\Omega^{\prime} \Subset \Omega$ and denote $T^{\prime}:=\mathbb{R}^{n}+i \bar{\Omega}^{\prime} \subset \mathbb{C}^{n}$. We endow $T^{\prime}$ with the Euclidean metric induced from $\mathbb{C}^{n}$.

We will need the following definition.

Definition 7.1. A function $f \in C\left(T^{\prime}\right)$ is called continuous almost periodic if the family of its translates $\left\{T^{\prime} \ni z \mapsto f(z+t)\right\}_{t \in \mathbb{R}^{n}}$ is relatively compact in $C_{b}\left(T^{\prime}\right)$ (the space of bounded continuous functions on $T^{\prime}$ endowed with sup-norm).

Proposition 7.2 (see, e.g., [1]). Any continuous almost periodic function on $T^{\prime}$ is bounded and uniformly continuous.

By $A P C\left(T^{\prime}\right)$ we denote the Banach algebra of continuous almost periodic functions on $T^{\prime}$ endowed with sup-norm.

We set

$$
p(z):=\left(e^{i z_{1}}, \ldots, e^{i z_{n}}\right), \quad z=\left(z_{1}, \ldots, z_{n}\right) \in T^{\prime}, \quad \text { and } \quad T_{0}^{\prime}:=p\left(T^{\prime}\right) .
$$

Then Banach algebra $C_{A P}\left(T^{\prime}\right), A P:=A P\left(\mathbb{Z}^{n}\right), \mathbb{Z}^{n} \cong p^{-1}\left(x_{0}\right)\left(x_{0} \in X_{0}\right)$, associated to covering $p: T^{\prime} \rightarrow T_{0}^{\prime}$ and endowed with sup-norm, is well defined (see Section 1).

To prove the theorem it suffices to show that $A P C\left(T^{\prime}\right)=C_{A P}\left(T^{\prime}\right)$. (Because the space of holomorphic almost periodic functions on $T$ consists of all functions in $\mathcal{O}(T)$ whose restrictions to each tube subdomain $T^{\prime} \subset T$ are in $A P C\left(T^{\prime}\right)$, and $\mathcal{O}_{A P}(T):=\mathcal{O}(T) \cap\left\{f \in C(T):\left.f\right|_{T^{\prime}} \in C_{A P}\left(T^{\prime}\right)\right.$ for each $\left.\left.T^{\prime} \subset T\right\}.\right)$

First, let $f \in A P C\left(T^{\prime}\right)$, i.e., for any sequence $\left\{t_{k}\right\} \subset \mathbb{R}^{n}$ there exists a subsequence of $\left\{T^{\prime} \ni z \mapsto f\left(z+t_{k_{l}}\right)\right\}$ that converges uniformly on $T^{\prime}$. In particular, it follows that for each fixed $z_{0} \in T^{\prime}$ and a sequence $\left\{d_{k}\right\} \subset \mathbb{Z}^{n}$ the family of translates $\left\{\mathbb{Z}^{n} \ni g \mapsto f\left(z_{0}+g+d_{k}\right)\right\}$ has a convergent subsequence which implies (since $C_{A P}\left(T^{\prime}\right)$ is a metric space) that it is relatively compact in the topology of uniform convergence on $\mathbb{Z}^{n}$. This means that the function $\mathbb{Z}^{n} \ni g \mapsto f\left(z_{0}+g\right)$ belongs to $A P\left(\mathbb{Z}^{n}\right)$. Also, by Proposition 7.2 function $f$ is bounded and uniformly continuous on $T^{\prime}$. Hence, by definition, $f \in C_{A P}\left(T^{\prime}\right)$.

Now, let $f \in C_{A P}\left(T^{\prime}\right)$. We must show that $f \in A P C\left(T^{\prime}\right)$. To this end we fix some sequence $\left\{t_{k}\right\} \subset \mathbb{R}^{n}$. Let $\mu: \mathbb{R}^{n} \rightarrow \mathbb{R}^{n} / \mathbb{Z}^{n}$ be the natural projection. Since $\mathbb{R}^{n} / \mathbb{Z}^{n}$ is compact, $\left\{\mu\left(t_{k}\right)\right\}$ has a convergent subsequence. We may assume without loss of generality that $\left\{\mu\left(t_{k}\right)\right\}$ itself converges and has limit 0 . Hence, there exists a sequence $\left\{d_{k}\right\} \subset \mathbb{Z}^{n}$ such that $\left|t_{k}-d_{k}\right| \rightarrow 0$ as $k \rightarrow \infty$. Since $f$ is uniformly continuous on $T^{\prime}$, functions

$$
h_{k}(z):=\left|f\left(z+t_{k}\right)-f\left(z+d_{k}\right)\right| \rightarrow 0 \quad \text { uniformly on } T^{\prime} \text { as } k \rightarrow \infty .
$$

Hence, it suffices to show that sequence $\left\{T^{\prime} \ni z \mapsto f\left(z+d_{k}\right)\right\}$ has a convergent subsequence.

Let $C:=\left\{z=\left(z_{1}, \ldots, z_{n}\right) \in T^{\prime}: 0 \leq \operatorname{Re}\left(z_{i}\right) \leq 1,1 \leq i \leq n\right\}$. Since $f \in$ $C_{A P}\left(T^{\prime}\right)$, for each fixed $w \in C$ the family of translates $\left\{\mathbb{Z}^{n} \ni g \mapsto f\left(w+g+d_{k}\right)\right\}$ 
is relatively compact in the topology of uniform convergence on $\mathbb{Z}^{n}$. Let $S \subset C$ be a countable dense subset. Using Cantor's diagonal argument we find a subsequence $\left\{d_{k_{l}}\right\}$ of $\left\{d_{k}\right\}$ such that for each $w \in S$ the family of translates $\left\{\mathbb{Z}^{n} \ni g \mapsto\right.$ $\left.f\left(w+g+d_{k_{l}}\right)\right\}$ converges in the topology of uniform convergence on $\mathbb{Z}^{n}$.

Now, since $f$ is uniformly continuous on $T^{\prime}$, for every $\varepsilon>0$ there exists $\delta>0$ such that for all $w_{1}, w_{2} \in C$ satisfying $\left|w_{1}-w_{2}\right|<\delta$ and all $h \in \mathbb{Z}^{n}$,

$$
\left|f\left(w_{1}+h\right)-f\left(w_{2}+h\right)\right|<\frac{\varepsilon}{3} .
$$

Since $C$ is compact, it can be covered by finitely many $\delta$-neighbourhoods of points, say, $w_{1}, \ldots, w_{p}$, in $S$. Then we can find $N \in \mathbb{N}$ so that for all $l, m>N, w_{j}$, $1 \leq j \leq p$, and $g \in \mathbb{Z}^{n}$,

$$
\left|f\left(w_{j}+g+d_{k_{l}}\right)-f\left(w_{j}+g+d_{k_{m}}\right)\right|<\frac{\varepsilon}{3} .
$$

The last two inequalities together with the triangle inequality imply that for all $l, m>N, z \in C$ and $g \in \mathbb{Z}^{n}$,

$$
\left|f\left(z+g+d_{k_{l}}\right)-f\left(z+g+d_{k_{m}}\right)\right|<\varepsilon .
$$

Since $\left\{z+g: z \in C, g \in \mathbb{Z}^{n}\right\}=T^{\prime}$, the latter implies that for all $l, m>N$ and $z \in T^{\prime}$

$$
\left|f\left(z+d_{k_{l}}\right)-f\left(z+d_{k_{m}}\right)\right|<\varepsilon .
$$

Thus $\left\{T^{\prime} \ni z \mapsto f\left(z+d_{k_{l}}\right)\right\}$ is a Cauchy sequence in the topology of uniform convergence on $T^{\prime}$, i.e., it converges uniformly on $T^{\prime}$.

The proof is complete.

\section{Proofs of Propositions 5.1, 5.5, 5.7, 5.9 and 5.15}

\subsection{Proof of Proposition 5.1}

The proof follows straightforwardly from (2.3) and the fact that $V$ is dense in $U$ (because $\iota(X)$ is dense in $c_{\mathfrak{a}} X$, cf. Section 2).

\subsection{Proof of Proposition 5.5}

According to Proposition 5.4, it suffices to prove coherence of the ideal sheaf $I_{Z}$ $\left(\subset \mathcal{O}_{V_{0} \times K}\right)$ of the complex submanifold $Z:=Z_{0} \times K$ of $V_{0} \times K$, where $V_{0} \subset X_{0}$ and $K \subset \hat{G}_{\mathfrak{a}}$ are open, $K \in \mathfrak{Q}$ is an element of the basis of topology $\mathfrak{Q}$ of $\hat{G}_{\mathfrak{a}}$ (see Subsection 5.2) and $Z_{0} \subset V_{0}$ is a complex submanifold. Here $\mathcal{O}_{V_{0} \times K}$ denotes the structure sheaf of $V_{0} \times K$ (see Subsection 5.1); also, by $\mathcal{O}_{V_{0}}$ we denote the structure sheaf of $V_{0}$ and by $I_{Z_{0}} \subset \mathcal{O}_{V_{0}}$ the ideal sheaf of $Z_{0} \subset V_{0}$.

By Cartan's theorem (see, e.g., [33]) every point in $V_{0}$ has a neighbourhood over which $I_{Z_{0}}$ has a free resolution. Replacing $V_{0}$ by a smaller subset, if necessary, we may assume without loss of generality that such resolution is defined over $V_{0}$ :

$$
0 \rightarrow \mathcal{O}_{V_{0}}^{m_{N}} \stackrel{\varphi_{N-1}}{\longrightarrow} \cdots \rightarrow \mathcal{O}_{V_{0}}^{m_{1}} \stackrel{\varphi_{0}}{\longrightarrow} I_{Z_{0}} \rightarrow 0 .
$$


Further, by the classical Cartan theorem B (see, e.g., [33]), every point in $V_{0}$ has a neighbourhood $U_{0} \subset V_{0}$ biholomorphic to an open polydisk in $\mathbb{C}^{n}$ such that the sequence of sections induced by (8.1)

$$
0 \rightarrow \Gamma\left(U_{0}, \mathcal{O}_{V_{0}}^{m_{N}}\right) \stackrel{\bar{\varphi}_{N-1}}{\longrightarrow} \cdots \rightarrow \Gamma\left(U_{0}, \mathcal{O}_{V_{0}}^{m_{1}}\right) \stackrel{\bar{\varphi}_{0}}{\longrightarrow} \Gamma\left(U_{0}, I_{Z_{0}}\right) \rightarrow 0
$$

is exact.

For an open subset $L \subset K, L \in \mathfrak{Q}$, by $C(L)$ we denote the Fréchet space of complex continuous functions on $L$ endowed with the topology of uniform convergence on compact subsets $N_{k} \subset L, k \in \mathbb{N}$, that form an exhaustion of $L$, i.e., $N_{k} \subset N_{k+1}$ for all $k$ and $\cup_{k \in \mathbb{N}} N_{k}=L$ (such sets exist by Lemma $7.4(1)$ in [15]). We endow the space $\mathcal{O}\left(U_{0} \times L\right)$ defined in Subsection 5.1 with the topology of uniform convergence on subsets $W_{0, k} \times N_{k}$, where $\left\{W_{0, k}\right\}_{k \in \mathbb{N}}$ is an exhaustion of $U_{0}$ by compact subsets, which makes it a Fréchet space. Then we have

$$
\Gamma\left(U_{0} \times L, \mathcal{O}_{V_{0} \times K}\right)=: \mathcal{O}\left(U_{0} \times L\right) \cong C(L) \otimes \mathcal{O}\left(U_{0}\right):=C(L) \otimes \Gamma\left(U_{0}, \mathcal{O}_{V_{0}}\right)
$$

where $\otimes$ stands for the completion of the symmetric tensor product in the corresponding Fréchet space.

Next, we may assume without loss of generality that $V_{0}$ is an open polydisk in $\mathbb{C}^{n}$ and $Z_{0}$ is the intersection of a complex subspace of $\mathbb{C}^{n}$ with $V_{0}$. Then using the Taylor series expansion of a holomorphic function on $U_{0} \times L$ vanishing on $Z_{0} \times K$ (i.e., an element of $\left.\Gamma\left(U_{0} \times L, I_{Z_{0} \times K}\right)\right)$, we easily obtain that

$$
\Gamma\left(U_{0} \times L, I_{Z_{0} \times K}\right) \cong C(L) \otimes \Gamma\left(U_{0}, I_{V_{0}}\right)
$$

By Theorem B in [16] the operation $\otimes$ is an exact functor. Thus, from (8.2), (8.3) and (8.4) we obtain that every point in $V_{0} \times K$ has a neighbourhood of the form $U_{0} \times L$ over which the sequence of sections

$$
0 \rightarrow \Gamma\left(U_{0} \times L, \mathcal{O}_{V_{0} \times K}^{m_{N}}\right) \stackrel{\hat{\varphi}_{N-1}}{\longrightarrow} \cdots \rightarrow \Gamma\left(U_{0} \times L, \mathcal{O}_{V_{0} \times K}^{m_{1}}\right) \stackrel{\hat{\varphi}_{0}}{\longrightarrow} \Gamma\left(U_{0} \times L, I_{Z_{0} \times K}\right) \rightarrow 0
$$

is exact, where morphisms $\hat{\varphi}_{i}$ are defined on the corresponding symmetric tensor products by the formula

$$
\hat{\varphi}_{i}\left(\sum_{i=1}^{l} f_{i} \otimes g_{i}\right)=\sum_{i=1}^{l} f_{i} \otimes \bar{\varphi}_{i}\left(g_{i}\right), \quad f_{i} \in C(L), \quad g_{i} \in \Gamma\left(U_{0}, \mathcal{O}_{V_{0}}^{m_{i+1}}\right),
$$

and then extended to $C(L) \otimes \Gamma\left(U_{0}, \mathcal{O}_{V_{0}}^{m_{i+1}}\right)$ by continuity. Hence, the sequence of sheaves generated by $(8.4)$

$$
0 \rightarrow \mathcal{O}_{V_{0} \times K}^{m_{N}} \rightarrow \cdots \rightarrow \mathcal{O}_{V_{0} \times K}^{m_{1}} \rightarrow I_{Z_{0} \times K} \rightarrow 0
$$

is exact. This shows that the sheaf $I_{Z}$ is coherent. 


\subsection{Proof of Proposition 5.7}

Let us prove the first assertion.

Let $Y \subset c_{\mathfrak{a}} X$ be the closure of $\iota(Z)$, where $Z \subset X$ is a complex a-submanifold, in $c_{\mathfrak{a}} X$. We fix a point $y \in Y$ and use notation of Definition 2.6. Since the open cover $\mathcal{V}$ in the definition of $Z$ is of class $\left(\mathcal{T}_{\mathfrak{a}}\right)$ (and, hence, is the pullback by $\iota$ of an open cover of $c_{\mathfrak{a}} X$, see Definition 2.5), there exist an open subset $V \in \mathcal{V}$, $V=\iota^{-1}(U)$ for an open neighbourhood $U \subset c_{\mathfrak{a}} X$ of $y$, and functions $h_{i} \in \mathcal{O}_{\mathfrak{a}}(V)$, $1 \leq i \leq k$, determining $Z \cap V$, i.e., satisfying conditions (1), (2) of Definition 2.6. By Proposition 5.1 there exist (uniquely determined) functions $\hat{h}_{i} \in \mathcal{O}(U)$ such that $h_{i}=\iota^{*} \hat{h}_{i}$ for all $i$. It follows from condition (2) of Definition 2.6 and the fact that $\iota(V)$ is dense in $U$ that functions $\hat{h}_{i}$ satisfy condition (2) of Definition 5.3 at points of $U \cap Y$. Therefore, since $y \in Y$ is arbitrary, to complete the proof it suffices to show that $U \cap Y=\hat{Y}_{U}$, where $\hat{Y}_{U} \subset U$ denotes the common zero locus of functions $\left.\hat{h}_{i}\right|_{U}, 1 \leq i \leq k$.

Indeed, using the argument of the proof of Proposition 5.4 and shrinking $U$, if necessary, we obtain that there exists a biholomorphism $\Phi \in \mathcal{O}\left(U_{0} \times K, U\right)$, where $U_{0} \subset X_{0}, K \subset \hat{G}_{\mathfrak{a}}$ are open, and a closed submanifold $Z_{0} \subset U_{0}$ such that $\Phi^{-1}\left(\hat{Y}_{U}\right)=Z_{0} \times K$ and $\Phi\left(U_{0} \times(K \cap j(G))\right)=U \cap \iota(X)$. In particular, since $h_{i}=\iota^{*} \hat{h}_{i}$ for all $i$, we have $\Phi\left(Z_{0} \times(K \cap j(G))\right)=U \cap \iota(Z)$. Hence, since $Z_{0} \times(K \cap j(G))$ is dense in $Z_{0} \times K$ (see subsection 2.1), $U \cap \iota(Z)$ is dense in $\hat{Y}_{U}$, i.e., $U \cap Y=\hat{Y}_{U}$, as required. The proof of the first assertion is complete.

The second assertion follows easily from Definitions 2.6, 5.3, and (2.3).

\subsection{Proof of Proposition 5.9}

First, let $f$ be a holomorphic a-function on $Z:=\iota^{-1}(Y)$ in the sense of Definition 2.8, i.e., there is a function $F \in C_{\mathfrak{a}}(X)$ such that $\left.F\right|_{Z}=f$. By Proposition 5.1 there exists a function $\hat{F} \in C\left(c_{\mathfrak{a}} X\right)$ such that $\iota^{*} \hat{F}=F$. We set $\hat{f}:=\left.\hat{F}\right|_{Y}$. Since $\iota^{*} \hat{f}=f$, we obtain $\hat{f} \in \mathcal{O}(Y)$ (see Definition 5.8), as required.

Now, let $\hat{f} \in \mathcal{O}(Y)$. Since $c_{\mathfrak{a}} X$ is a normal space, by the Tietze-Urysohn extension theorem there exists a function $\hat{F} \in C\left(c_{\mathfrak{a}} X\right)$ such that $\left.\hat{F}\right|_{Y}=\hat{f}$. By definition (cf. (2.3)) $F:=\iota^{*} \hat{F}$ belongs to $C_{\mathfrak{a}}(X)$. Since $\left.F\right|_{Z}=f$, function $f$ $\left(=\iota^{*} \hat{f}\right)$ is a holomorphic $\mathfrak{a}$-function on $Z$ in the sense of Definition 2.8.

\subsection{Proof of Proposition $\mathbf{5 . 1 5}$}

For a point $x \in Y$, consider its open neighbourhood $V$ for which there exists a biholomorphic map $\varphi: V \rightarrow Z_{0} \times K$, where $Z_{0} \subset \mathbb{C}^{p}$ is an open ball and $K \subset \hat{G}_{\mathfrak{a}}$ is open (see Proposition 5.4). We choose an open neighbourhood $W \Subset V$ of $x$ so that $\varphi(W)=Z_{0}^{\prime} \times K^{\prime}$, where $Z_{0}^{\prime} \Subset Z_{0}$ is an open ball of the same center as $Z_{0}$ and $K^{\prime} \Subset K$ is an open subset. Then under the identification of $V$ with $Z_{0} \times K$ by $\varphi$ the restriction to $W$ of the space of $C^{\infty} \bar{\partial}$-closed $(p, k+1)$-forms on $V$ is identified with a subspace of the space of $C^{\infty} \bar{\partial}$-closed $(p, k+1)$-forms on $Z_{0}^{\prime}$ with values in the Banach space $C_{b}\left(K^{\prime}\right)$ of bounded continuous functions on $K$ endowed with 
sup-norm (see Subsection 5.5 for the corresponding definitions). According to Lemma 6.1, such Banach-valued forms on $Z_{0}^{\prime}$ are $\bar{\partial}$-exact. This completes the proof of the proposition.

\section{Proofs of Theorems $2.7,2.9$ and 2.10}

\subsection{Proof of Theorem 2.7}

Our proof is based on Theorem 2.3 and the equivalence of notions of a complex a-submanifold of $X$ and a complex submanifold of $c_{\mathfrak{a}} X$ (see Subsection 5.3 for the corresponding definitions and results).

Thus, it suffices to prove that given a complex submanifold $Y \subset c_{\mathfrak{a}} X$ of codimension $k$ there exists an at most countable collection of functions $f_{i} \in \mathcal{O}\left(c_{\mathfrak{a}} X\right)$, $i \in I$, such that

(i) $Y=\left\{y \in c_{\mathfrak{a}} X: f_{i}(y)=0\right.$ for all $\left.i \in I\right\}$, and

(ii) for each $y_{0} \in Y$ there exist a neighbourhood $W=\hat{\Pi}\left(W_{0}, L\right)$ (see (5.2) for notation) and functions $f_{i_{1}}, \ldots, f_{i_{k}}$ such that $Y \cap W=\left\{y \in U: f_{i_{1}}(y)=\cdots=\right.$ $\left.f_{i_{k}}(y)=0\right\}$ and the rank of map $z \mapsto\left(f_{1}(z, \omega), \ldots, f_{k}(z, \omega)\right),(z, \omega) \in W$, is maximal at each point of $Y \cap W$.

By Proposition 5.5 the ideal sheaf $I_{Y}$ of $Y$ is coherent, hence by Theorem 2.3, there exists an at most countable collection of sections $f_{i} \in \Gamma\left(c_{\mathfrak{a}} X, I_{Y}\right)\left(\subset \mathcal{O}_{c_{\mathfrak{a}} X}\right)$, $i \in I$, that generate $I_{Y}$ at each point of $c_{\mathfrak{a}} X$. (This collection is at most countable because any open cover of $c_{\mathfrak{a}} X$ admits an at most countable refinement as fibres of the bundle $\bar{p}: c_{\mathfrak{a}} X \rightarrow X_{0}$ are compact and any open cover of complex manifold $X_{0}$ admits an at most countable refinement.) Therefore condition (i) is valid for this collection of functions. In addition, for every point $y_{0} \in Y$ there exist a neighbourhood $U=\hat{\Pi}\left(U_{0}, K\right)$ of $y_{0}$, sections $f_{i_{1}}, \ldots, f_{i_{m}}$ and functions $u_{j l} \in \mathcal{O}\left(c_{\mathfrak{a}} X\right)$, $1 \leq j \leq k, 1 \leq l \leq m$, such that

$$
h_{j}=u_{j 1} f_{i_{1}}+\cdots+u_{j m} f_{i_{m}}, \quad 1 \leq j \leq k,
$$

where $h_{j}$ are generators of $\left.I_{Y}\right|_{U}$ from Definition 5.3 (modulo a biholomorphic transformation of Proposition 5.4 we may identify $h_{j}$ with $z_{j}$, the $j$-th coordinate of $\left.z \in \mathbb{C}^{n}\right)$.

Equation (9.1) implies that $Y \cap U=\left\{y \in U: f_{i_{1}}(y)=\cdots=f_{i_{m}}(y)=0\right\}$.

Next, let $\nabla h_{j}, \nabla f_{i_{l}}$ denote the vector-valued functions $\nabla_{z} h_{j}(z, \omega), \nabla_{z} f_{i_{l}}(z, \omega)$, $(z, \omega) \in U$. Then

$$
\nabla h_{j}=u_{j 1} \nabla f_{i_{1}}+\cdots+u_{j m} \nabla f_{i_{m}} \quad \text { on } Y \cap U, \quad 1 \leq j \leq k .
$$

Since $\left(\nabla h_{j}\right)_{j=1}^{k}$ has rank $k$ on $U$, we obtain that $k \leq m$, and $\left(u_{j l}\right)_{1 \leq j \leq k, 1 \leq l \leq m}$, $\left(\nabla f_{i_{l}}\right)_{l=1}^{m}$ have rank $k$ at each point of $U$. Thus there exist two subfamilies of vector-valued functions $\left(u_{j l_{1}}\right)_{j=1}^{k}, \ldots,\left(u_{j l_{k}}\right)_{j=1}^{k}$ and $\nabla \tilde{f}_{l_{1}}, \ldots, \nabla \tilde{f}_{l_{k}}, \tilde{f}_{l}:=f_{i_{l}}$, that are linearly independent at $y_{0}$. Now, we apply the holomorphic inverse function theorem (see Theorem 6.2) to the matrix identity (9.1) to find a neighbourhood 
$W=\hat{\Pi}\left(W_{0}, L\right) \Subset U$ of $y_{0}$ such that functions $\left.\tilde{f}_{l}\right|_{W}, l \neq l_{i}, 1 \leq i \leq k$, belong to the ideal in $\mathcal{O}(W)$ generated by $\left.\tilde{f}_{l_{1}}\right|_{W}, \ldots,\left.\tilde{f}_{l_{k}}\right|_{W}$, and the rank of map $z \mapsto$ $\left(\tilde{f}_{l_{1}}(z, \omega), \ldots, \tilde{f}_{l_{k}}(z, \omega)\right),(z, \omega) \in W$, is maximal at each point of $Y \cap W$. Clearly, $Y \cap W=\left\{y \in U: \tilde{f}_{l_{1}}(y)=\cdots=\tilde{f}_{l_{k}}(y)=0\right\}$.

This completes the proof of the theorem.

\subsection{Proof of Theorem 2.9}

The proof follows the lines of the proof of the classical tubular neighbourhood theorem (see, e.g., [26]).

We use notation and results of Section 5. Clearly, Theorem 2.9 is a corollary of:

Theorem 9.1. Let $X_{0}$ be a Stein manifold, and $Y \subset c_{\mathfrak{a}} X$ a complex submanifold (see Subsection 5.3). Then there exists an open neighbourhood $\Omega \subset c_{\mathfrak{a}} X$ of $Y$ and maps $h_{t} \in \mathcal{O}(\Omega, \Omega)$ continuously depending on $t \in[0,1]$, such that $\left.h_{t}\right|_{Y}=\operatorname{Id}_{Y}$ for all $t \in[0,1], h_{0}=\operatorname{Id}_{\Omega}$ and $h_{1}(\Omega)=Y$.

Proof. In the proof of Theorem 9.1 we use the following notation and definitions.

Let $U$ be an open subset of $c_{\mathfrak{a}} X$ or of a complex submanifold $Y \subset c_{\mathfrak{a}} X$. In the category of ringed spaces $\left(U, \mathcal{O}_{U}\right)$ (see Subsections 5.1, 5.3) we define in a standard way holomorphic vector bundles on $U$, their subbundles, the Whitney sum of bundles, holomorphic bundle morphisms, etc (see [37] for similar definitions).

Now, we define the (holomorphic) tangent bundle $T c_{\mathfrak{a}} X$ on $c_{\mathfrak{a}} X$ as the pullback $\bar{p}^{*} T X_{0}$ of the (holomorphic) tangent bundle $T X_{0}$ of $X_{0}$. We denote by $T_{x} c_{\mathfrak{a}} X$ the fibre of $T c_{\mathfrak{a}} X$ at $x \in c_{\mathfrak{a}} X$.

Next, we define a Hermitian metric on $T c_{\mathfrak{a}} X$ as the pullback by $\bar{p}$ of a (complete) Hermitian metric on $T X_{0}$.

Let $Y \subset c_{\mathfrak{a}} X$ be a complex submanifold. Every point $x \in Y$ has a neighbourhood $U=\hat{\Pi}\left(U_{0}, K\right) \subset c_{\mathfrak{a}} X$, where $U_{0} \subset X_{0}, K \subset \hat{G}_{\mathfrak{a}}$ are open, so that $Y \cap U$ is the set of common zeros of functions $h_{1}, \ldots, h_{k} \in \mathcal{O}(U)$ such that the maximum of moduli of determinants of square submatrices of the Jacobian matrix of the map $z \mapsto\left(h_{1}(z, \omega), \ldots, h_{k}(z, \omega)\right),(z, \omega) \in U$, is uniformly bounded away from zero (see Definition 5.3). We define the tangent bundle $T Y$ of $Y$ as the subbundle of $\left.T c_{\mathfrak{a}} X\right|_{Y}$ whose fibres are orthogonal to the local vector fields $(z, \omega) \mapsto D_{z} h_{1}(z, \omega), \ldots, D_{z} h_{k}(z, \omega),(z, \omega) \in Y \cap U$. Namely, in local coordinates $(z, \omega) \in U$ the metric has a form

$$
d s^{2}(z, \omega)=\sum_{l, j} g_{l j}(z) d z_{l} \otimes d \bar{z}_{j}, \quad g_{l j}(z):=\left(\frac{\partial}{\partial z_{l}}, \frac{\partial}{\partial z_{j}}\right)_{(z, \omega)} ;
$$

hence, if vector fields $D_{z} h_{i}(1 \leq i \leq k)$ are given by

$$
D_{z} h_{i}(z, \omega)=\sum_{l} a_{l i}(z, \omega) \frac{\partial}{\partial z_{l}}
$$

then $T_{(z, \omega)} Y$ consists of vectors $\sum_{l} b_{l} \frac{\partial}{\partial z_{l}}$ such that $\sum_{l, j} a_{l i}(z, \omega) \bar{b}_{j} g_{l j}(z)=0$ for all $1 \leq i \leq k$. 
It is easily seen that $\iota^{*} T Y$ coincides with the holomorphic tangent bundle of the complex submanifold $\iota^{-1}(Y) \subset X$. Since $\iota\left(\iota^{-1}(Y)\right)$ is dense in $Y$, the bundle $T Y$ is uniquely defined by the latter condition (see Subsection 5.5).

Consequently, we obtain the notion of the (holomorphic) normal bundle $N Y \subset$ $\left.T c_{\mathfrak{a}} X\right|_{Y}$ of $Y$.

The tangent bundle $T X_{0}$ is generated by finitely many holomorphic vector fields $V_{0, k}, 1 \leq k \leq m$, which determine holomorphic local flows $\varphi_{k}: O_{k} \rightarrow X_{0}$, where $O_{k}$ is an open neighbourhood of $\{0\} \times X_{0}$ in $\mathbb{C} \times X_{0}$ such that the differential of the map

$$
F_{0}(t, \cdot):=\left(\varphi_{m}\left(s_{m}, \cdot\right) \circ \cdots \circ \varphi_{1}\left(s_{1}, \cdot\right)\right): X_{0} \rightarrow X_{0}, \quad t=\left(s_{1}, \ldots, s_{m}\right),
$$

at $t=0$ is non-degenerate. The map $F_{0}$ is defined and holomorphic in a neighbourhood $W_{0}$ of $\{0\} \times X_{0}$ in $\mathbb{C}^{m} \times X_{0}$.

We will need notation and results of Section 4 in [15]. There, we have established that $c_{\mathfrak{a}} X$ (as a set) is the disjoint union of connected complex manifolds $X_{H}$ $(H \in \Upsilon)$ each is a covering of $X_{0}$. Using the lifting property, for every $H \in \Upsilon$ we can lift $F_{0}$ to a unique map $\tilde{F}_{H}(t, \cdot): W_{H} \rightarrow X_{H}$ that is defined and holomorphic on the neighbourhood $W_{H}:=\left(\operatorname{Id}_{\mathbb{C}^{m}} \times p_{H}\right)^{-1}\left(W_{0}\right)$ of $\{0\} \times X_{H}$ in $\mathbb{C}^{m} \times X_{H}$, where $p_{H}: X_{H} \rightarrow X_{0}$ is the covering projection. It is not difficult to show that these maps constitute a holomorphic map

$$
\tilde{F}: W \rightarrow c_{\mathfrak{a}} X
$$

where $W:=\left(\operatorname{Id}_{\mathbb{C}^{m}} \times \bar{p}\right)^{-1}\left(W_{0}\right)$ is a neighbourhood of $\{0\} \times c_{\mathfrak{a}} X$ in $\mathbb{C}^{m} \times c_{\mathfrak{a}} X$. (Alternatively, one can define the map $\tilde{F}$ using the covering homotopy theorem and the local structure of $c_{\mathfrak{a}} X$, see Subsection 5.1. Note that in local coordinates lifted from $X_{0}$ the map $\tilde{F}$ looks exactly the same as $F_{0}$.)

Next, for a fixed $x \in c_{\mathfrak{a}} X$ we consider a linear map

$$
\theta_{x}=\left.\partial_{t}\right|_{t=0} \tilde{F}(t, x): \mathbb{C}^{m} \rightarrow T_{x} c_{\mathfrak{a}} X .
$$

We denote by $\theta$ the corresponding holomorphic bundle morphism $\mathbb{C}^{m} \times c_{\mathfrak{a}} X \rightarrow$ $T c_{\mathfrak{a}} X$.

Since vector fields $\bar{p}^{*} V_{0, j}$ span $T c_{\mathfrak{a}} X$, the maps $\theta_{x}$ are surjective, for every $x \in c_{\mathfrak{a}} X$. Since $\left.T Y \subset T c_{\mathfrak{a}} X\right|_{Y}$, we can define a holomorphic vector bundle over $Y$,

$$
E^{\prime}:=\theta^{*}(T Y) \subset Y \times \mathbb{C}^{m}
$$

Lemma 9.2. There exists a holomorphic vector bundle $E$ over $Y$ such that

$$
E^{\prime} \oplus E=Y \times \mathbb{C}^{m}
$$

Proof. We have an exact sequence of holomorphic vector bundles over $Y$

$$
0 \rightarrow E^{\prime} \rightarrow Y \times \mathbb{C}^{m} \stackrel{q}{\rightarrow} E^{\prime \prime} \rightarrow 0
$$


(here $E^{\prime \prime}$ is the quotient bundle) which induces an exact sequence of Čech cohomology groups with values in the sheaves of germs of holomorphic sections of the corresponding holomorphic vector bundles

$$
\begin{aligned}
0 \rightarrow \Gamma\left(Y, \operatorname{Hom}_{\mathcal{O}}\left(E^{\prime \prime}, E^{\prime}\right)\right) & \rightarrow \Gamma\left(Y, \operatorname{Hom}_{\mathcal{O}}\left(E^{\prime \prime}, Y \times \mathbb{C}^{m}\right)\right) \rightarrow \Gamma\left(Y, \operatorname{Hom}_{\mathcal{O}}\left(E^{\prime \prime}, E^{\prime \prime}\right)\right) \\
& \stackrel{\delta}{\rightarrow} H^{1}\left(Y, \operatorname{Hom}_{\mathcal{O}}\left(E^{\prime \prime}, E^{\prime}\right)\right) \rightarrow \cdots .
\end{aligned}
$$

Recall that sequence (9.2) splits if and only if $\delta(I)=0$, where $I: E^{\prime \prime} \rightarrow E^{\prime \prime}$ is the identity homomorphism (see, e.g., Ch.1,4.1d-f in [37]). Since the sheaf $\operatorname{Hom}_{\mathcal{O}}\left(E^{\prime \prime}, E^{\prime}\right)$ is locally free, Theorem 5.12 implies that $H^{1}\left(Y, \operatorname{Hom}_{\mathcal{O}}\left(E^{\prime \prime}, E^{\prime}\right)\right)=0$. Hence there is a holomorphic homomorphism $u: E^{\prime \prime} \rightarrow Y \times \mathbb{C}^{m}$ such that $q \circ u=\mathrm{Id}$, i.e., $Y \times \mathbb{C}^{m}=E^{\prime} \oplus E$ with $E:=u\left(E^{\prime \prime}\right)$.

It follows from this lemma that the restriction $\theta:\left.E \rightarrow T c_{\mathfrak{a}} X\right|_{E}$ is an injective holomorphic bundle morphism such that $\left.T c_{\mathfrak{a}} X\right|_{Y}=T Y \oplus \theta(E)$. Therefore, we have:

Lemma 9.3. $\left.\theta\right|_{E}$ determines a holomorphic isomorphism between the bundles $E$ and $N Y$.

Further, we define

$$
F:=\tilde{F} \circ\left(\left.\theta\right|_{E}\right)^{-1}: N Y \rightarrow c_{\mathfrak{a}} X
$$

and show that there exists a neighbourhood $V$ of the zero section of $N Y$ that is mapped by $F$ biholomorphically to a neighbourhood of $Y$ in $c_{\mathfrak{a}} X$. Using this and replacing $V$ by a smaller neighbourhood of the zero section of $N Y$ with convex fibres over $Y$, we can define the required maps $h_{t}$ by dilations along images of the fibres of this smaller neighbourhood under $F$. This would complete the proof of the theorem.

We prove that $F$ is a biholomorphism near the zero section of $N Y$ in two steps.

(1) First, we show that $F$ is a local biholomorphism, i.e., every point of the zero section of $N Y$ has a neighbourhood $V$ such that the restriction $\left.F\right|_{V}$ determines a biholomorphism between $V$ and $F(V) \subset c_{\mathfrak{a}} X$.

Indeed, by Proposition 5.4 for every $x \in Y$ one can find its neighbourhood $U_{x} \subset c_{\mathfrak{a}} X$ biholomorphic to $U_{0} \times K$, where $U_{0} \subset X_{0}, K \subset \hat{G}_{\mathfrak{a}}$ are open, such that (a) $Y \cap U$ is biholomorphic to $Y_{0} \times K$ for $Y_{0} \subset U_{0}$ a complex submanifold of $U_{0}$; (b) $\left.N Y\right|_{Y \cap U} \cong N Y_{0} \times K$; (c) $F: N Y_{0} \times K \rightarrow U_{0} \times K$ is determined by a collection of maps $N Y_{0} \rightarrow U_{0}$ continuously depending on variable in $K$ such that the maximum of moduli of determinants of square submatrices of their Jacobian matrices are uniformly bounded away from zero.

The required result now follows from the inverse function theorem with continuous dependence on parameter (Theorem 6.2).

(2) Now, we show that there is a neighbourhood $V$ of the zero section of $N Y$ such that $\left.F\right|_{V}$ is an injection; since $F$ is holomorphic, this would imply the required.

We have defined $F$ in such a way that it maps the fibres of $E$ that lie over the points of $Y \cap X_{H}$ into $X_{H}$, for every $H \in \Upsilon$ (see the definition of $\tilde{F}$ above). 
Let $\rho_{H}$ be the path metric determined by the pullback to $X_{H}$ of the (complete) Hermitian metric on $X_{0}$ (that we fixed previously). We define a pseudo-metric $\rho$ on $c_{\mathfrak{a}} X$ by

$$
\rho\left(x_{1}, x_{2}\right):=\rho_{H}\left(x_{1}, x_{2}\right)<\infty \text { if } x_{1}, x_{2} \in X_{H}, \quad \rho\left(x_{1}, x_{2}\right):=\infty \text { otherwise. }
$$

Let $\|\cdot\|_{x}$ denote the norm on the fibres of bundle $N Y$ determined by the restriction to $N Y$ of the Hermitian metric on $T c_{\mathfrak{a}} X$, defined above. For $y \in Y$ by $v_{y}$ we denote an element of $N_{y} Y$. For $x \in Y$ we set

$$
V_{\delta}(x):=\left\{v_{y} \in N Y: \rho(x, y)<\delta,\left\|v_{y}\right\|_{y}<\delta\right\} .
$$

Using the construction of part (1) based on the inverse function theorem with continuous dependence on parameter, one can easily show that there is a positive function $b \in C(Y)$ such that

$$
\rho\left(x, F\left(v_{x}\right)\right) \leq b(x)\left\|v_{x}\right\|_{x}
$$

for all $v_{x}$ in a neighbourhood of the zero section of $N Y$. Also, using the assertion of part (1), one can show that there is a positive function $r \in C(Y)$ such that $\left.F\right|_{V_{r(x)}(x)}$ is a biholomorphism for all $x \in Y$. Now, we set

$$
V:=\left\{v_{y} \in N Y:\left\|v_{y}\right\|_{y}<\frac{r(y)}{2 \max \{1, b(y)\}}\right\}
$$

This is an open neighbourhood of the zero section of $N Y$. Let us show that $\left.F\right|_{V}$ is injective. Indeed, assume that $v_{x}, v_{y} \in V$ and $F\left(v_{x}\right)=F\left(v_{y}\right)$. Without loss of generality we may assume that $r(y) \leq r(x)$. Thus, using the triangle inequality and (9.3) we obtain:

$$
\begin{aligned}
\rho(x, y) & \leq \rho\left(x, F\left(v_{x}\right)\right)+\rho\left(F\left(v_{x}\right), F\left(v_{y}\right)\right)+\rho\left(y, F\left(v_{y}\right)\right) \\
& =\rho\left(x, F\left(v_{x}\right)\right)+\rho\left(y, F\left(v_{y}\right)\right) \leq \frac{1}{2} r(x)+\frac{1}{2} r(y) \leq r(x) .
\end{aligned}
$$

It follows that $v_{x}, v_{y} \in V_{r(x)}(x)$. Since $\left.F\right|_{V_{r(x)}(x)}$ is a biholomorphism, we arrive to a contradiction with the assumption $F\left(v_{x}\right)=F\left(v_{y}\right)$. Therefore, $\left.F\right|_{V}$ is injective.

The proof of the theorem is complete.

Remark 9.4. In the classical tubular neighbourhood theorem the neighbourhood of a closed submanifold is chosen to be a Stein open submanifold (see, e.g. [26]). The following question naturally arises: is it possible to choose $\Omega$ in Theorem 9.1 to be a Stein open submanifold of $c_{\mathfrak{a}} X$ (see the definition in Subsection 5.4)?

\subsection{Proof of Theorem $\mathbf{2 . 1 0}$}

In view of Propositions 5.7 and 5.9, and (2.3), Theorem 2.10 follows from:

Theorem 9.5. Let $X_{0}$ be a Stein manifold, $Y \subset c_{\mathfrak{a}} X$ be a complex submanifold, $f \in \mathcal{O}(Y)$. Then there exists a function $F \in \mathcal{O}\left(c_{\mathfrak{a}} X\right)$ such that $\left.F\right|_{Y}=f$. 
Proof. We will need:

Lemma 9.6. Let $f \in \mathcal{O}(Y)$. For every point $y_{0} \in Y$, there exist a neighbourhood $V \subset c_{\mathfrak{a}} X$ of $y_{0}$ and a function $F_{V} \in \mathcal{O}(V)$ such that $\left.F_{V}\right|_{V \cap Y}=\left.f\right|_{V \cap Y}$.

Proof of Lemma 9.6. By Proposition 5.4, there exist an open neighbourhood $V \subset$ $c_{\mathfrak{a}} X$ of $y_{0}$, open subsets $V_{0} \subset \mathbb{C}^{n}, K \subset \hat{G}_{\mathfrak{a}}$, and a biholomorphic map $\Phi \in \mathcal{O}\left(V_{0} \times\right.$ $K, V)$ such that

$\Phi^{-1}(V \cap Y)=Z_{0} \times K, \quad$ where $Z_{0}=\left\{\left(0, \ldots, 0, z_{k+1}, \ldots, z_{n}\right):\left(z_{1}, \ldots, z_{n}\right) \in V_{0}\right\}$.

Let $\tilde{f}:=\Phi^{*} f \in \mathcal{O}\left(Z_{0} \times K\right)$. We define

$$
\tilde{F}_{V}\left(z_{1}, \ldots, z_{n}, \omega\right):=\tilde{f}\left(z_{k+1}, \ldots, z_{n}, \omega\right), \quad\left(z_{1}, \ldots, z_{n}, \omega\right) \in V_{0} \times K,
$$

and $F_{V}:=\left(\Phi^{-1}\right)^{*} \tilde{F}_{V}$.

Now, by Lemma 9.6 there exist an open cover $\mathcal{U}=\left\{U_{j}\right\}$ of $c_{\mathfrak{a}} X$ and functions $f_{j} \in \mathcal{O}\left(U_{j}\right)$ such that $\left.f_{j}\right|_{Y \cap U_{j}}=\left.f\right|_{Y \cap U_{j}}$ if $Y \cap U_{j} \neq \varnothing$; if $Y \cap U_{j}=\varnothing$, we define $f_{j}:=0$. Then $\left\{g_{i j}:=f_{i}-f_{j}\right.$ on $\left.U_{i} \cap U_{j} \neq \varnothing\right\}$ is a 1-cocycle with values in sheaf $I_{Y}$ of ideals of $Y$. By Proposition 5.5 sheaf $I_{Y}$ is coherent, so by Theorem 2.4 $H^{1}\left(c_{\mathfrak{a}} X, I_{Y}\right)=0$. Thus, $\left\{g_{i j}\right\} \mid \mathcal{V}$ represents 0 in $H^{1}\left(\mathcal{V}, I_{Y}\right)$ for a refinement $\mathcal{V}$ of $\mathcal{U}$. To avoid abuse of notation we may assume without loss of generality that $\mathcal{V}=\mathcal{U}$. Therefore, we can find holomorphic functions $h_{j} \in \Gamma\left(U_{j}, I_{Y}\right)$ such that $g_{i j}=h_{i}-h_{j}$ on $U_{i} \cap U_{j} \neq \varnothing$. Now, we define $\left.F\right|_{U_{j}}:=f_{j}-h_{j}$ for all $j$.

\section{Proofs of Theorems 2.17, 2.18, 2.20 and Proposition 2.19}

In the proofs we use the following results.

(1) Let $\left\{U_{\alpha}\right\}$ be an open cover of $Z \subset X$ and $L$ and $L^{\prime}$ be line bundles on $Z$ in one of the categories introduced in Subsection 2.3 defined on $\left\{U_{\alpha}\right\}$ by cocycles $d_{\alpha \beta}$ and $d_{\alpha \beta}^{\prime}$, respectively. Recall that an isomorphism between $L$ and $L^{\prime}$ is given by nowhere zero functions $h_{\alpha}$ on $U_{\alpha}$ (of the same category as $d_{\alpha \beta}, d_{\alpha \beta}^{\prime}$ ) such that $d_{\alpha \beta}^{\prime}=h_{\alpha} d_{\alpha \beta} h_{\beta}^{-1}$ on $U_{\alpha} \cap U_{\beta}$ for all $\alpha, \beta$.

(2) In the proofs below we work with the Čech cohomology groups of sheaves on $X$ or complex a-submanifolds of $X$ associated to presheaves of functions defined on subsets of $X$ open in topology $\mathcal{T}_{\mathfrak{a}}$ or their intersections with the submanifolds. By definition (see, e.g., [33]), these groups are inverse limits of the Čech cohomology groups defined on open covers of class $\left(\mathcal{T}_{\mathfrak{a}}\right)$ of $X$ or of its complex $\mathfrak{a}$-submanifolds (see Definitions 2.5 and 2.12).

(3) Recall (cf. (2.3)) that $\mathcal{O}_{\mathfrak{a}}(V)=\iota^{*} \mathcal{O}(U)$, where $V=\iota^{-1}(U) \in \mathcal{T}_{\mathfrak{a}}, U \subset c_{\mathfrak{a}} X$ are open. In particular, $\mathcal{O}_{\mathfrak{a}}=\iota^{*} \mathcal{O}$, where $\mathcal{O}$ is the structure sheaf of $c_{\mathfrak{a}} X$, and $\iota: X \rightarrow c_{\mathfrak{a}} X$ is the canonical map (see Section 2). Since $\iota(X)$ is dense in $c_{\mathfrak{a}} X$, spaces $\mathcal{O}_{\mathfrak{a}}(V)$ and $\mathcal{O}(U)$ are isomorphic. It follows from the definition of cohomology groups that $H^{p}\left(X, \mathcal{O}_{\mathfrak{a}}\right)=H^{p}\left(c_{\mathfrak{a}} X, \mathcal{O}\right), p \in \mathbb{N}$. A similar argument yields $H^{p}\left(Z, \mathcal{O}_{\mathfrak{a}}\right)=H^{p}(Y, \mathcal{O}), p \in \mathbb{N}$, where $Y \subset c_{\mathfrak{a}} X$ is a complex submanifold and $Z:=\iota^{-1}(Y) \subset X$. 


\subsection{Proof of Theorem 2.17}

Let us prove the first assertion.

Let $E=\left\{f_{\alpha} \in \mathcal{O}_{\mathfrak{a}}\left(U_{\alpha}\right)\right\}$ so that $L_{E}$ is determined by the cocycle $\left\{d_{\alpha \beta}:=\right.$ $\left.f_{\alpha} f_{\beta}^{-1} \in \mathcal{O}_{\mathfrak{a}}\left(U_{\alpha} \cap U_{\beta}\right)\right\}$. By Definition 2.16 there exist nowhere zero functions $h_{\alpha} \in \mathcal{O}_{\ell_{\infty}}\left(U_{\alpha}\right)$ with $\left|h_{\alpha}\right| \in C_{\mathfrak{a}}\left(U_{\alpha}\right)$ such that $d_{\alpha \beta}=h_{\alpha}^{-1} h_{\beta}$ for all $\alpha, \beta$, see (1) in the beginning of Section 10. We define $f:=f_{\alpha} h_{\alpha}$ on $U_{\alpha}$. This is a function in $\mathcal{O}(Z)$ such that $|f|_{U_{\alpha}}|=| f_{\alpha}|| h_{\alpha} \mid \in C_{\mathfrak{a}}\left(U_{\alpha}\right)$ for each $\alpha$. One can easily show using a partition of unity on the complex submanifold $Y \subset c_{\mathfrak{a}} X$ such that $Z=\iota^{-1}(Y)$ (see Subsection 5.3) that the latter implies $|f| \in C_{\mathfrak{a}}(Z)$. By our construction, divisor $E_{f} \in \operatorname{Div}(X)$ is $\ell_{\infty}$-equivalent to $E$.

Conversely, suppose that $\mathfrak{a}$ is such that $\hat{G}_{\mathfrak{a}}$ is a compact topological group and $j(G) \subset \hat{G}_{\mathfrak{a}}$ is a dense subgroup, and let $E=\left\{f_{\alpha} \in \mathcal{O}_{\mathfrak{a}}\left(U_{\alpha}\right)\right\} \in \operatorname{Div}_{\mathfrak{a}}(X)$. By our assumption there exist nowhere zero functions $h_{\alpha} \in \mathcal{O}_{\ell_{\infty}}\left(U_{\alpha}\right)$ with $h_{\alpha}^{-1} \in$ $\mathcal{O}_{\ell_{\infty}}\left(U_{\alpha}\right)$ such that $\left.f\right|_{U_{\alpha}}=h_{\alpha} f_{\alpha}$ for all $\alpha$ (see Definition 2.15). It is clear that the family $\left\{h_{\alpha}\right\}$ determines an $\ell_{\infty}$-isomorphism of the line a-bundle $L_{E}:=\left\{\left(U_{\alpha} \cap\right.\right.$ $\left.\left.U_{\beta}, d_{\alpha \beta}:=f_{\alpha} f_{\beta}^{-1}\right)\right\}$ of $E$ onto the trivial bundle $\left\{\left(U_{\alpha} \cap U_{\beta}, 1\right)\right\}$ (see (1) in the beginning of Section 10); to conclude that $L_{E}$ is a-semi-trivial it remains to show that $\left|h_{\alpha}\right|,\left|h_{\alpha}\right|^{-1} \in C_{\mathfrak{a}}\left(U_{\alpha}\right)$ for all $\alpha$ (see Definition 2.16).

By Proposition 5.1 there exist open subsets $V_{\alpha} \subset c_{\mathfrak{a}} X$ and functions $\hat{f}_{\alpha} \in \mathcal{O}\left(V_{\alpha}\right)$ such that $U_{\alpha}=\iota^{-1}\left(V_{\alpha}\right)$ and $f_{\alpha}=\iota^{*} \hat{f}_{\alpha}$ for all $\alpha$; also, there exists a function $F \in C\left(c_{\mathfrak{a}} X\right)$ such that $|f|=\iota^{*} F$. We will show that there exist positive functions $g_{\alpha} \in C\left(V_{\alpha}\right)$ such that $\iota^{*} g_{\alpha}=\left|h_{\alpha}\right|$. Then by $(2.3)\left|h_{\alpha}\right|,\left|h_{\alpha}\right|^{-1} \in C_{\mathfrak{a}}\left(U_{\alpha}\right)$.

Let us fix $\alpha$. First, note that since the required inclusion is a local property, we may assume without loss of generality that $V_{\alpha}$ is biholomorphic to $V_{0} \times K$, where $V_{0} \subset X_{0}$ is an open coordinate chart and $K \subset \hat{G}_{\mathfrak{a}}$ is open, $K \in \mathfrak{Q}$ (see Subsection 5.1). In particular, we can identify $V_{\alpha}$ with $V_{0} \times K$.

The proof of the required inclusion consists of three parts.

(1) Let us show that $\hat{f}_{\alpha}(\cdot, \eta) \not \equiv 0$ for every $\eta \in K$.

By definition, the family of functions $\hat{f}:=\left\{\hat{f}_{\alpha}\right\}$ determines a not identically zero holomorphic section of a holomorphic line bundle $\hat{L}_{E}$ on $c_{\mathfrak{a}} X$ (see Subsection 2.3). Based on results in [15] we have:

$c_{\mathfrak{a}} X=\sqcup_{H \in \Upsilon} \iota_{H}\left(X_{H}\right)$, where $X_{H}=X, \iota_{H}: X_{H} \rightarrow c_{\mathfrak{a}} X$ is holomorphic (see Subsection 5.1) and $\iota_{H}\left(X_{H}\right)$ is dense in $c_{\mathfrak{a}} X$ for every $H \in \Upsilon$ (cf. Subsection 4.1 and Example 4.2 in [15]).

In particular, since $\iota_{H}\left(X_{H}\right)$ is dense in $c_{\mathfrak{a}} X$, assuming that section $\hat{f} \equiv 0$ on $\iota_{H}\left(X_{H}\right)$ for some $H \in \Upsilon$ we obtain $\hat{f} \equiv 0$ on $c_{\mathfrak{a}} X$, a contradiction.

Suppose, on the contrary, that $\hat{f}_{\alpha}(\cdot, \eta) \equiv 0$ for some $\eta \in K$. Then there exists a unique $H \in \Upsilon$ such that $V_{0} \times\{\eta\}\left(\cong \hat{\Pi}\left(V_{0},\{\eta\}\right)\right) \subset \iota_{H}\left(X_{H}\right)$. We set $\hat{f}_{\eta}:=\iota_{H}^{*} \hat{f}$. This is a holomorphic section of holomorphic line bundle $\iota_{H}^{*} \hat{L}_{E}$ on $X_{H}$. By our assumption $\hat{f}_{\eta}$ is zero on an open subset of $X_{H}(=X)$. Since $\hat{f}_{\eta}$ is holomorphic and $X_{H}$ is connected, $\hat{f}_{\eta} \equiv 0$; hence $\left.\hat{f}\right|_{\iota_{H}\left(X_{H}\right)} \equiv 0$, a contradiction, i.e., $\hat{f}_{\alpha}(\cdot, \eta) \not \equiv 0$.

(2) Next, we show that $\left.F\right|_{V_{\alpha}}(\cdot, \eta) \not \equiv 0$ for every $\eta \in K$.

Assume on the contrary that there exists $\eta_{0} \in K$ such that $\left.F\right|_{V_{\alpha}}(\cdot, \eta) \equiv 0$. 
By part $(1), \hat{f}_{\alpha}\left(\cdot, \eta_{0}\right) \not \equiv 0$, so we can choose an open $V_{0}^{\prime} \Subset V_{0}$ such that $\left|\hat{f}_{\alpha}\left(\cdot, \eta_{0}\right)\right| \geq$ $c>0$ on $V_{0}^{\prime}$. Now, under the identification $V_{\alpha}$ with $V_{0} \times K$ the set $U_{\alpha}=\iota^{-1}\left(V_{\alpha}\right)$ is identified with $U_{\alpha}=V_{0} \times L$, where $L:=j^{-1}(K) \subset G$, and so $\left.\iota\right|_{U_{\alpha}}=\operatorname{Id}_{V_{0}} \times\left. j\right|_{L}$. Since $\left.F\right|_{V_{\alpha}}$ is continuous and $j(L)$ is dense in $K$ (see Subsection 2.1), there exists a net $\left\{g_{\gamma}\right\} \subset L$ such that the net $\left\{j\left(g_{\gamma}\right)\right\} \subset K$ converges to $\eta_{0}$. By continuity $\hat{f}_{\alpha}\left(\cdot, j\left(g_{\gamma}\right)\right)$ converges to $\hat{f}_{\alpha}\left(\cdot, \eta_{0}\right)$ uniformly on $V_{0}^{\prime}$ (so we may assume without loss of generality that $\left|\hat{f}_{\alpha}\left(\cdot, j\left(g_{\gamma}\right)\right)\right| \geq c / 2>0$ for all $\gamma$ ), while $\left.F\right|_{V_{\alpha}}\left(\cdot, j\left(g_{\gamma}\right)\right)$ converges to 0 uniformly on $V_{0}^{\prime}$. Since $|f|_{U_{\alpha}}|=| h_{\alpha}|| f_{\alpha} \mid$, where $|f|=\iota^{*} F, f_{\alpha}=\iota^{*} \hat{f}$, the latter implies that $\left|h_{\alpha}\left(\cdot, g_{\gamma}\right)\right| \rightarrow 0$ uniformly over $V_{0}^{\prime}$. We will show that this leads to a contradiction with our assumption.

Indeed, due to results of Subsection 4.1 of [15] there exists an equivariant with respect to right actions of $G$ continuous proper map $\kappa: \hat{G}_{\ell_{\infty}} \rightarrow \hat{G}_{\mathfrak{a}}$. Set $K^{\prime}:=\kappa^{-1}(K)$. Passing to the corresponding subnets, if necessary, we may assume without loss of generality that there exists a net $\left\{\xi_{\gamma}\right\} \subset K^{\prime}$ having limit $\xi_{0} \in K^{\prime}$ such that $\kappa\left(\xi_{0}\right)=\eta_{0}$ and $\kappa\left(\xi_{\gamma}\right)=j\left(g_{\gamma}\right)$ for all $\gamma$. Further, since by our assumption $h_{\alpha} \in \mathcal{O}_{\ell_{\infty}}\left(U_{\alpha}\right)$, by Proposition 5.1 there exists a function $\tilde{h}_{\alpha} \in \mathcal{O}\left(V_{0} \times K^{\prime}\right)$ such that $\left(\operatorname{Id}_{V_{0}} \times j_{\ell_{\infty}}\right) * \tilde{h}_{\alpha}=h_{\alpha}$ (see Subsection 2.1 for notation). Now, since $\left|h_{\alpha}\left(\cdot, g_{\gamma}\right)\right| \rightarrow 0$ uniformly on $V_{0}^{\prime}$, we obtain that $\left|\tilde{h}_{\alpha}\left(\cdot, \xi_{\gamma}\right)\right| \rightarrow 0$ uniformly on $V_{0}^{\prime}$; so by continuity $\tilde{h}_{\alpha}\left(\cdot, \xi_{0}\right) \equiv 0$ on $V_{0}^{\prime}$. However, by (2.3) function $h_{\alpha}^{-1} \in \mathcal{O}_{\ell_{\infty}}\left(U_{\alpha}\right)$ admits a continuous extension to $V_{0} \times K^{\prime}$ such that its product with $\tilde{h}_{\alpha}$ is identically 1 (because $h_{\alpha} h_{\alpha}^{-1} \equiv 1$ on $U_{\alpha}$ and $\left(\operatorname{Id}_{V_{0}} \times j_{\ell_{\infty}}\right)\left(U_{\alpha}\right)$ is dense in $\left.V_{0} \times K^{\prime}\right)$. This contradicts the identity $\tilde{h}_{\alpha}\left(\cdot, \xi_{0}\right) \equiv 0$ on $V_{0}^{\prime}$ and completes the proof of step (2).

(3) Finally, we show that there exists a positive function $g_{\alpha} \in C\left(V_{\alpha}\right), V_{\alpha}=$ $V_{0} \times K$, such that $\iota^{*} g_{\alpha}=\left|h_{\alpha}\right|$.

Let $\mathcal{Z} \subset V_{\alpha}$ be the union of zero loci of functions $\left.F\right|_{V_{\alpha}}$ and $\left|\hat{f}_{\alpha}\right|$. By parts (1) and (2), we obtain that the open set $\mathcal{Z}^{c}:=V_{\alpha} \backslash \mathcal{Z}$ is dense in $V_{\alpha}$ and, moreover, $\left.F\right|_{V_{\alpha}} /\left|\hat{f}_{\alpha}\right|$ and $\left|\hat{f}_{\alpha}\right| /\left.F\right|_{V_{\alpha}}$ are continuous on $\mathcal{Z}^{c}$. We set $\tilde{\kappa}:=\left(\operatorname{Id}_{V_{0}} \times \kappa\right): V_{0} \times \hat{G}_{\ell_{\infty}} \rightarrow$ $V_{0} \times \hat{G}_{\mathfrak{a}}$. By the definition pullbacks by $\tilde{\kappa}$ of $\left(F /\left|\hat{f}_{\alpha}\right|\right) \mid \mathcal{Z}^{c}$ and $\left(\left|\hat{f}_{\alpha}\right| / F\right) \mid \mathcal{Z}^{c}$ to $\tilde{\kappa}^{-1}\left(\mathcal{Z}^{c}\right) \subset V_{0} \times K^{\prime}$ coincide with $\left|\tilde{h}_{\alpha}\right|$ and $\left|\tilde{h}_{\alpha}\right|^{-1}$ there (see part (2)). This, the fact that the open set $\tilde{\kappa}^{-1}\left(\mathcal{Z}^{c}\right)$ is dense in $V_{0} \times K^{\prime}$ and the definition of $\tilde{\kappa}$ imply that $\left|\tilde{h}_{\alpha}\right|$ is constant on fibres of $\tilde{\kappa}$. Since $\tilde{\kappa}$ is a proper continuous map and $V_{0} \times K$, $V_{0} \times K^{\prime}$ are locally compact Hausdorff spaces, the latter implies that there exists a positive function $g_{\alpha} \in C\left(V_{0} \times K\right)$ such that $\tilde{\kappa}^{*} g_{\alpha}=\left|\tilde{h}_{\alpha}\right|$. By the definition $g_{\alpha}=\left.F\right|_{V_{\alpha}} /\left|\hat{f}_{\alpha}\right|$ on $\mathcal{Z}^{c}$. This yields $\iota^{*} g_{\alpha}=\left|h_{\alpha}\right|$, as required.

\subsection{Proof of Theorem 2.18}

Suppose that conditions (1) and (2) are satisfied. Let us show that the holomorphic line a-bundle $L$ is $\mathfrak{a}$-semi-trivial.

Suppose that $L$ is defined by a holomorphic 1-cocycle $\left\{c_{\alpha \beta}\right\}$ on an open cover $\left\{U_{\alpha}\right\}$ of $Z$ of class $\left(\mathcal{T}_{\mathfrak{a}}\right)$. In what follows, we may need to pass several times to refinements of class $\left(\mathcal{T}_{\mathfrak{a}}\right)$ of the cover $\left\{U_{\alpha}\right\}$. To avoid abuse of notation we may assume without loss of generality that $\left\{U_{\alpha}\right\}$ is acyclic with respect to the corresponding sheaves so that according to the classical Leray lemma we can work only with cover $\left\{U_{\alpha}\right\}$. 
By (1) we can find functions $c_{\alpha} \in \mathcal{O}_{\mathfrak{a}}\left(U_{\alpha}\right)$ such that $c_{\alpha}^{-1} \in \mathcal{O}_{\mathfrak{a}}\left(U_{\alpha}\right)$ and $c_{\alpha}^{-1} c_{\alpha \beta} c_{\beta}=d_{\alpha \beta}$ is locally constant on $U_{\alpha} \cap U_{\beta}$ for all $\alpha, \beta$; hence, $\left\{d_{\alpha \beta}\right\}$ determines an equivalent discrete $\mathfrak{a}$-bundle $L^{\prime}$ on $Z$. Now, we have polar representation

$$
d_{\alpha \beta}=\left|d_{\alpha \beta}\right| e^{i l_{\alpha \beta}} \quad \text { for all } \alpha, \beta .
$$

Then $\left\{\left|d_{\alpha \beta}\right|\right\} \in Z^{1}\left(\left\{U_{\alpha}\right\}, \mathbb{R}_{+}\right),\left\{e^{i l_{\alpha \beta}}\right\} \in Z^{1}\left(\left\{U_{\alpha}\right\}, \mathbb{U}_{1}\right)$, where $\mathbb{U}_{1}$ is the 1-dimensional unitary group, are multiplicative locally constant cocycles.

Since $\left|d_{\alpha \beta}\right| \neq 0$ are locally constant and belong to $\mathcal{O}_{\mathfrak{a}}\left(U_{\alpha} \cap U_{\beta}\right)$, functions $\log \left|d_{\alpha \beta}\right| \in \mathcal{O}_{\mathfrak{a}}\left(U_{\alpha} \cap U_{\beta}\right)$ as well and form an additive holomorphic 1-cocyle on $\left\{U_{\alpha}\right\}$. We can resolve this cocycle by Theorem 5.12 (see definitions in the beginning of Section 10), i.e., there exist functions $g_{\alpha} \in \mathcal{O}_{\mathfrak{a}}\left(U_{\alpha}\right)$ such that $e^{g_{\alpha}} \cdot e^{-g_{\beta}}=\left|d_{\alpha \beta}\right|$ for all $\alpha, \beta$.

Further, by condition (2) bundle $L^{\prime}$ is trivial in the category of discrete line bundles on $Z$. This implies existence of functions $e^{i l_{\alpha}} \in \mathcal{O}\left(U_{\alpha}\right)$, where $l_{\alpha}$ are real-valued locally constant, such that

$$
e^{i l_{\alpha \beta}}=e^{i l_{\alpha}} \cdot e^{-i l_{\beta}} \quad \text { on } \quad U_{\alpha} \cap U_{\beta} .
$$

Now, we define

$$
\psi_{\alpha}:=e^{-g_{\alpha}} \cdot e^{-i l_{\alpha}} \cdot c_{\alpha} \in \mathcal{O}_{\ell_{\infty}}\left(U_{\alpha}\right) .
$$

Then $d_{\alpha \beta}=\psi_{\alpha} \psi_{\beta}^{-1}$, so the family of functions $\left\{\psi_{\alpha}\right\}$ determines an isomorphism in category $\mathcal{L}_{\ell_{\infty}}(Z)$ of $L$ onto the trivial line bundle (see (1) in the beginning of Section 10). Moreover, $\left|\psi_{\alpha}\right|=e^{-\operatorname{Re} g_{\alpha}}\left|c_{\alpha}\right|,\left|\psi_{\alpha}\right|^{-1}=e^{\operatorname{Re} g_{\alpha}}\left|c_{\alpha}^{-1}\right| \in C_{\mathfrak{a}}\left(U_{\alpha}\right)$ for all $\alpha$, as required.

Conversely, suppose that the holomorphic line $\mathfrak{a}$-bundle $L$ is $\mathfrak{a}$-semi-trivial. Let us show that conditions (1) and (2) are satisfied. As before, we assume that $L$ is determined by a cocycle $\left\{c_{\alpha \beta} \in \mathcal{O}_{\mathfrak{a}}\left(U_{\alpha} \cap U_{\beta}\right)\right\}$ on cover $\left\{U_{\alpha}\right\}$ of $Z$. By Definition 2.16 there exist nowhere zero functions $\psi_{\alpha} \in \mathcal{O}\left(U_{\alpha}\right)$ with $\left|\psi_{\alpha}\right|,\left|\psi_{\alpha}\right|^{-1} \in C_{\mathfrak{a}}\left(U_{\alpha}\right)$ such that $\psi_{\alpha} c_{\alpha \beta} \psi_{\beta}^{-1} \equiv 1$ on $U_{\alpha} \cap U_{\beta} \neq \emptyset$ (see (1) in the beginning of Section 10).

We will use notation and results of Subsection 5.5. Denote

$$
\Lambda_{\mathfrak{a}}^{p, k}\left(U_{\alpha}\right):=\iota^{*} \Lambda^{p, k}\left(V_{\alpha}\right), \quad Z_{\mathfrak{a}}^{p, k}\left(U_{\alpha}\right):=\iota^{*} Z^{p, k}\left(V_{\alpha}\right)
$$

where $V_{\alpha} \subset Y$ is open and such that $U_{\alpha}=\iota^{-1}\left(V_{\alpha}\right), Y \subset c_{\mathfrak{a}} X$ is the closure of $\iota(Z)$ (a complex submanifold of $c_{\mathfrak{a}} X$, see Proposition 5.7), $\Lambda^{p, k}(Y)$ is the space of $(p, k)$-forms on $Y$ and $Z^{p, k}(Y)$ is the space of $\bar{\partial}$-closed form on $Y$. Also, denote $C_{\mathfrak{a}}^{\infty}\left(U_{\alpha}\right):=\iota^{*} C^{\infty}\left(V_{\alpha}\right)$.

Let us show that $\left|\psi_{\alpha}\right|,\left|\psi_{\alpha}\right|^{-1} \in C_{\mathfrak{a}}^{\infty}\left(U_{\alpha}\right)$. We may assume without loss of generality that $U_{\alpha}=U_{0} \times j^{-1}(K), V_{\alpha}=U_{0} \times K$, where $U_{0} \subset \mathbb{C}^{m}, m:=\operatorname{dim}_{\mathbb{C}} Z$, is an open ball and $K \subset \hat{G}_{\mathfrak{a}}, K \in \mathfrak{Q}$, see (5.3), is open (see Proposition 5.4 and Subsection 5.1). Then there exist $\tilde{\psi}_{\alpha}, \tilde{\psi}_{\alpha}^{-1} \in \mathcal{O}\left(U_{0} \times K^{\prime}\right), K^{\prime}:=\kappa^{-1}(K) \subset \hat{G}_{\ell_{\infty}}$, such that $\left(\operatorname{Id}_{U_{0}} \times j_{\ell_{\infty}}\right)^{*}\left(\tilde{\psi}_{\alpha}\right)^{ \pm 1}=\left(\psi_{\alpha}\right)^{ \pm 1}$ (see part $(2)$ in the proof of Theorem 2.17 and Subsection 5.1) which can be viewed as holomorphic functions on $U_{0}$ taking values in the Fréchet space $C\left(K^{\prime}\right)$. In particular, these are $C\left(K^{\prime}\right)$-valued $C^{\infty}$ functions. Further, since $\left|\psi_{\alpha}\right|,\left|\psi_{\alpha}\right|^{-1} \in C_{\mathfrak{a}}\left(U_{\alpha}\right)$, there exist nowhere zero functions $\hat{\psi}_{\alpha}$, 
$\hat{\psi}_{\alpha}^{-1} \in C\left(U_{0} \times K\right)$ whose pullbacks by $\operatorname{Id}_{U_{0}} \times \kappa$ to $U_{0} \times K^{\prime}$ coincide with $\tilde{\psi}_{\alpha}$ and $\tilde{\psi}_{\alpha}^{-1}$, respectively. The last two facts imply easily that $\hat{\psi}_{\alpha}, \hat{\psi}_{\alpha}^{-1} \in C^{\infty}\left(U_{0} \times K\right)$ (see Subsection 5.5 for the definition). Pullbacks of $\hat{\psi}_{\alpha}, \hat{\psi}_{\alpha}^{-1}$ by $\iota:=\operatorname{Id}_{U_{0}} \times j$ are functions $\left|\psi_{\alpha}\right|,\left|\psi_{\alpha}\right|^{-1}$. Thus these functions are in $C_{\mathfrak{a}}^{\infty}\left(U_{\alpha}\right)$.

Now, since $\hat{\psi}_{\alpha}, \hat{\psi}_{\alpha}^{-1}$ are nowhere zero, $\log \left|\psi_{\alpha}\right| \in C_{\mathfrak{a}}^{\infty}\left(U_{\alpha}\right)$; hence, forms $\partial\left(\log \left|\psi_{\alpha}\right|\right)$ belong to $\Lambda_{\mathfrak{a}}^{1,0}\left(U_{\alpha}\right)$ and satisfy $\bar{\partial} \partial\left(\log \left|\psi_{\alpha}\right|\right)=0$, that is, $\partial\left(\log \left|\psi_{\alpha}\right|\right) \in Z_{\mathfrak{a}}^{1,0}\left(U_{\alpha}\right)$. Identifying $U_{\alpha}$ with $U_{0} \times j^{-1}(K)$ by a biholomorphism (see Proposition 5.4) we obtain that $\partial\left(\log \left|\psi_{\alpha}\right|\right)$ is the pullback by $\iota$ of the $d$-closed holomorphic 1-form $\partial\left(\log \hat{\psi}_{\alpha}\right)$ on $U_{0}$ with values in the Fréchet space $C(K)$ (see Subsection 5.5 for notation). Integrating the latter form along rays in $U_{0}$ emanating from the center and taking the pullback of the obtained function by $\iota$ we obtain a function $u_{\alpha} \in$ $\mathcal{O}_{\mathfrak{a}}\left(U_{\alpha}\right)$ such that $\partial u_{\alpha}=\partial\left(\log \left|\psi_{\alpha}\right|\right)$. Hence, $\log \left|\psi_{\alpha}\right|-u_{\alpha}=\bar{v}_{\alpha}$ for some $v_{\alpha} \in$ $\mathcal{O}_{\mathfrak{a}}\left(U_{\alpha}\right)$. We define $b_{\alpha}:=u_{\alpha}+v_{\alpha} \in \mathcal{O}_{\mathfrak{a}}\left(U_{\alpha}\right)$. Then $\log \left|\psi_{\alpha}\right|=\operatorname{Re} b_{\alpha}$. Now, set

$$
d_{\alpha \beta}:=e^{b_{\alpha}} c_{\alpha \beta} e^{-b_{\beta}} \in \mathcal{O}_{\mathfrak{a}}\left(U_{\alpha} \cap U_{\beta}\right) \quad \text { for all } \alpha, \beta .
$$

Then $\left|d_{\alpha \beta}\right|=\left|\psi_{\alpha} c_{\alpha \beta} \psi_{\beta}^{-1}\right| \equiv 1$, i.e., $\left\{d_{\alpha \beta}\right\}$ is a locally constant 1-cocycle on the cover $\left\{U_{\alpha}\right\}$ of $Z$ with values in the unitary group $\mathbb{U}_{1}$. Therefore condition (1) is satisfied.

Further, $\psi_{\alpha} e^{-b_{\alpha}} d_{\alpha \beta} \psi_{\beta}^{-1} e^{b_{\beta}} \equiv 1$ for all $\alpha, \beta$ and $\left|\psi_{\alpha} e^{-b_{\alpha}}\right| \equiv 1$ on $U_{\alpha}$, that is, functions $\psi_{\alpha} e^{-b_{\alpha}}$ are locally constant for all $\alpha$. Hence the discrete line $\mathfrak{a}$-bundle $L^{\prime}:=\left\{\left(U_{\alpha} \cap U_{\beta}, d_{\alpha \beta}\right)\right\}$ is trivial in the category of discrete line bundles on $Z$, i.e., condition (2) is satisfied as well.

The proof of the theorem is complete.

\subsection{Proof of Proposition 2.19}

We use notation and results of Subsection 5.5. Suppose that algebra $\mathfrak{a}$ is selfadjoint. Then $Z=\iota^{-1}(Y)$ for a complex submanifold $Y$ of $\subset c_{\mathfrak{a}} X$ (see Definition 2.6 and Subsection 5.3). We set $\Lambda_{\mathfrak{a}}^{p, k}(Z):=\iota^{*} \Lambda^{p, k}(Y), Z_{\mathfrak{a}}^{p, k}(Z):=\iota^{*} Z^{p, k}(Y)$, and define

$$
H_{\mathfrak{a}}^{p, k}(Z):=Z_{\mathfrak{a}}^{p, k}(Z) / \bar{\partial} \Lambda_{\mathfrak{a}}^{p, k-1}(Z), \quad p \geq 0, k \geq 1, \quad H_{\mathfrak{a}}^{p, 0}(Z):=Z_{\mathfrak{a}}^{p, 0}(Z) .
$$

These spaces of forms and cohomology groups are isomorphic to their counterparts on $Y$, so we have analogues of Proposition 5.15 and Corollaries 5.16, 5.17 on $Z$ (see (2) and (3) in the beginning of Section 10).

We will also need an analogue of the de Rham complex on $Y$.

Let $Z^{m}(Y) \subset \Lambda^{m}(Y)$ denote the subspace of $d$-closed forms. Define

$$
\begin{aligned}
H^{m}(Y) & :=Z^{m}(Y) / d \Lambda^{m-1}(Y), \quad p \geq 0, \quad m \geq 1, \\
H^{0}(Y) & :=Z^{0}(Y) .
\end{aligned}
$$

("de Rham cohomology groups of $Y$ "). Now, set $\Lambda_{\mathfrak{a}}^{m}(Z):=\iota^{*} \Lambda^{m}(Y), Z_{\mathfrak{a}}^{m}(Z):=$ $\iota^{*} Z^{m}(Y)$,

$$
H_{\mathfrak{a}}^{m}(Z):=Z_{\mathfrak{a}}^{m-1}(Y) / d \Lambda_{\mathfrak{a}}^{m}(Z), \quad p \geq 0, m \geq 1, \quad H_{\mathfrak{a}}^{0}(Z):=Z_{\mathfrak{a}}^{0}(Z) .
$$

Then $H_{\mathfrak{a}}^{m}(Z)$ and $H^{m}(Y)$ are isomorphic. 
Let us denote by $\mathcal{O}_{\mathfrak{a}}$ the sheaf associated to the presheaf of functions $\mathcal{O}_{\mathfrak{a}}(U)$, $U \subset Z, U \in \mathcal{T}_{\mathfrak{a}}$ (see Definition 2.12). Let $\mathbb{Z}_{\mathfrak{a}}, \mathbb{R}_{\mathfrak{a}} \subset \mathcal{O}_{\mathfrak{a}}$ denote subsheaves of locally constant functions with values in groups $\mathbb{Z}, \mathbb{R}$, respectively. Using an argument similar to that of the proof of Proposition 5.15, where instead of Lemma 6.1 we use the Poincaré $d$-lemma for Banach-valued $d$-closed forms on a ball (see Subsection 8.5), one obtains an analogue of the $d$-Poincaré lemma on $Y$ (i.e., a $d$-closed $C^{\infty} m$-form, $m \geq 1$, on an open subset of $Y$ is locally $d$-exact). Then since sheaves $\Lambda^{m}$ of germs of $C^{\infty} m$-forms on $Y$ are fine, see Lemma 5.14 , by a standard result about cohomology groups of sheaves admitting acyclic resolutions (see, e.g., Ch. B, §1.3, of [34]), we obtain

$$
H_{\mathfrak{a}}^{m}(Z) \cong H^{m}\left(Z, \mathbb{R}_{\mathfrak{a}}\right), \quad m \geq 0 .
$$

Finally, by $\mathcal{O}_{\mathfrak{a}}^{*} \subset \mathcal{O}_{\mathfrak{a}}$ we denote a multiplicative subsheaf associated to the presheaf of functions $f \in \mathcal{O}_{\mathfrak{a}}(U), U \subset Z, U \in \mathcal{T}_{\mathfrak{a}}$, such that $f^{-1} \in \mathcal{O}_{\mathfrak{a}}(U)$ as well.

Proof of Proposition 2.19. First, we show that condition (1) of Theorem 2.18 is satisfied.

We have an exact sequence of sheaves

$$
0 \rightarrow \mathbb{Z}_{\mathfrak{a}} \rightarrow \mathcal{O}_{\mathfrak{a}} \stackrel{e^{2 \pi i}}{\rightarrow} \mathcal{O}_{\mathfrak{a}}^{*} \rightarrow 0
$$

which induces an exact sequence of cohomology groups

$$
\cdots \rightarrow H^{1}\left(Z, \mathbb{Z}_{\mathfrak{a}}\right) \rightarrow H^{1}\left(Z, \mathcal{O}_{\mathfrak{a}}\right) \rightarrow H^{1}\left(Z, \mathcal{O}_{\mathfrak{a}}^{*}\right) \stackrel{\delta}{\rightarrow} H^{2}\left(Z, \mathbb{Z}_{\mathfrak{a}}\right) \rightarrow \cdots
$$

By definition the class of holomorphic $\mathfrak{a}$-bundles isomorphic to the line $\mathfrak{a}$-bundle $L:=L_{E}$ of a divisor $E \in \operatorname{Div}_{\mathfrak{a}}(Z)$ determines an element of group $H^{1}\left(Z, \mathcal{O}_{\mathfrak{a}}^{*}\right)$; its image under $\delta$ in $H^{2}\left(Z, \mathbb{Z}_{\mathfrak{a}}\right)$ is denoted by $\delta(L)$ and is called the Chern class of $L$. On a suitable open cover $\left\{U_{\alpha}\right\}$ of $Z$ of class $\left(\mathcal{T}_{\mathfrak{a}}\right)$ element $\delta(L)$ is defined by a locally constant 2-cocycle $\left\{m_{\alpha \beta \gamma}^{L}\right\} \in Z^{2}\left(\left\{U_{\alpha}\right\}, \mathbb{Z}_{\mathfrak{a}}\right)$ given by the formula (see, e.g., [30])

$$
m_{\alpha \beta \gamma}^{L}=\frac{1}{2 \pi i}\left(\log c_{\alpha \beta}+\log c_{\beta \gamma}+\log c_{\gamma \alpha}\right) \quad \text { on } \quad U_{\alpha} \cap U_{\beta} \cap U_{\gamma},
$$

where $L$ is determined on $\left\{U_{\alpha}\right\}$ by 1-cocycle $\left\{c_{\alpha \beta} \in \mathcal{O}_{\mathfrak{a}}^{*}\left(U_{\alpha} \cap U_{\beta}\right)\right\}$.

Let $c(L)$ denote the image of $\delta(L)$ in $H^{2}\left(Z, \mathbb{R}_{\mathfrak{a}}\right)$ under the natural homomorphism $H^{2}\left(Z, \mathbb{Z}_{\mathfrak{a}}\right) \rightarrow H^{2}\left(Z, \mathbb{R}_{\mathfrak{a}}\right)$. We identify the last group with $H_{\mathfrak{a}}^{2}(Z)$, see (10.1). Since $\operatorname{dim}_{\mathbb{C}} Z=1$, element $c(L)$ is determined by a $d$-closed $(1,1)$-form $\eta \in Z_{\mathfrak{a}}^{2}(Z)$.

Lemma 10.1. $\eta=d \lambda$ for some $\lambda \in \Lambda_{\mathfrak{a}}^{1}(Z)$.

Proof. Since $Z$ is 1-dimensional, $\bar{\partial} \eta=0$. Hence, by the analogue of Corollary 5.17 on $Z$ we have $\eta=\bar{\partial} \lambda$ for some $\lambda \in \Lambda_{\mathfrak{a}}^{1,0}(Z)$. We have $\partial \lambda=0$, as $\Lambda_{\mathfrak{a}}^{2,0}(Z)=0$, so $d \lambda=(\bar{\partial}+\partial) \lambda=\bar{\partial} \lambda=\eta$, as required. 
The lemma implies that $c(L)=0$. Replacing cover $\left\{U_{\alpha}\right\}$ by its refinement of class $\left(\mathcal{T}_{\mathfrak{a}}\right)$, if necessary, we may assume without loss of generality that there exists a locally constant 1-cochain $\left\{s_{\alpha \beta} \in C_{\mathfrak{a}}\left(U_{\alpha} \cap U_{\beta}, \mathbb{R}_{\mathfrak{a}}\right)\right\}$ on $\left\{U_{\alpha}\right\}$ such that, for all $\alpha, \beta, \gamma$,

$$
m_{\alpha \beta \gamma}^{L}=s_{\alpha \beta}+s_{\beta \gamma}+s_{\gamma \alpha} \quad \text { on } \quad U_{\alpha} \cap U_{\beta} \cap U_{\gamma} .
$$

Then $\left\{\log c_{\alpha \beta}-2 \pi i \cdot s_{\alpha \beta}\right\} \in Z^{1}\left(\left\{U_{\alpha}\right\}, \mathcal{O}_{\mathfrak{a}}\right)$. According to Theorem 2.4 this cocycle represents 0 in $H^{1}\left(Z, \mathcal{O}_{\mathfrak{a}}\right)$ (as $H^{1}\left(Z, \mathcal{O}_{\mathfrak{a}}\right)=H^{1}(Y, \mathcal{O})$, see discussion in the beginning of Section 10). Again, passing to a refinement of cover $\left\{U_{\alpha}\right\}$ of class $\left(\mathcal{T}_{\mathfrak{a}}\right)$, if necessary, we may assume without loss of generality that this cocycle can be resolved on $\left\{U_{\alpha}\right\}$, that is, there exist $h_{\alpha} \in \mathcal{O}_{\mathfrak{a}}\left(U_{\alpha}\right)$ such that

$$
\log c_{\alpha \beta}-2 \pi i \cdot s_{\alpha \beta}=h_{\alpha}-h_{\beta} \quad \text { on } U_{\alpha} \cap U_{\beta} .
$$

We set $d_{\alpha \beta}:=e^{-h_{\alpha}} c_{\alpha \beta} e^{h_{\beta}}$ on $U_{\alpha} \cap U_{\beta}$. Then cocycle $\left\{d_{\alpha \beta}\right\}$ determines a discrete line a-bundle $L^{\prime}$ isomorphic to $L$. Therefore, condition (1) of Theorem 2.18 is satisfied.

Now, we show that under the additional hypothesis $H^{1}(Z, \mathbb{C})=0$ condition $(2)$ of Theorem 2.18 is satisfied as well.

First, note that $H^{2}(Z, \mathbb{Z})=0$. Indeed, $Z$ is a complex submanifold of a Stein manifold $X$, and hence itself is a Stein manifold. Therefore, since $\operatorname{dim}_{\mathbb{C}} Z=1$, $Z$ is homotopically equivalent to a 1-dimensional $\mathrm{CW}$-complex, which implies the required.

A discrete line bundle on $Z$ is determined (up to an isomorphism in the corresponding category) by an element of group $H^{1}\left(Z, \mathbb{C}^{*}\right)$, where $\mathbb{C}^{*}:=\mathbb{C} \backslash\{0\}$. Therefore, to show that the discrete line a-bundle $L^{\prime}$ is trivial in the category of discrete bundles on $Z$, it suffices to show that $H^{1}\left(Z, \mathbb{C}^{*}\right)=0$. In turn, the exact sequence of locally constant sheaves $0 \rightarrow \mathbb{Z} \rightarrow \mathbb{C} \stackrel{\text { exp }}{\rightarrow} \mathbb{C}^{*} \rightarrow 0$ on $Z$ induces an exact sequence of cohomology groups

$$
\cdots \rightarrow H^{1}(Z, \mathbb{Z}) \rightarrow H^{1}(Z, \mathbb{C}) \rightarrow H^{1}\left(Z, \mathbb{C}^{*}\right) \rightarrow H^{2}(Z, \mathbb{Z}) \rightarrow \cdots .
$$

Since $H^{1}(Z, \mathbb{C})=H^{2}(Z, \mathbb{Z})=0$, group $H^{1}\left(Z, \mathbb{C}^{*}\right)=0$, as required.

\subsection{Proof of Theorem 2.20}

Since $X_{0}$ is homotopy equivalent to open subset $Y_{0} \subset X_{0}, \pi_{1}\left(X_{0}\right)=\pi_{1}\left(Y_{0}\right)$ and the space $c_{\mathfrak{a}} X$ is homotopy equivalent to open subset $c_{\mathfrak{a}} Y \subset c_{\mathfrak{a}} X, Y:=p^{-1}\left(Y_{0}\right) \subset X$ (for $\mathfrak{a}=\ell_{\infty}$ the proof is given in Proposition 4.2 of [10]; the proof in the general case repeats it word by word).

We retain notation of Subsection 10.3. For the exact sequence of locally constant sheaves on $X$

$$
0 \rightarrow \mathbb{Z}_{\mathfrak{a}} \rightarrow \mathcal{O}_{\mathfrak{a}} \stackrel{e^{2 \pi i}}{\rightarrow} \mathcal{O}_{\mathfrak{a}}^{*} \rightarrow 0
$$

consider the induced exact sequences of cohomology groups

$$
\cdots \rightarrow H^{1}\left(X, \mathbb{Z}_{\mathfrak{a}}\right) \rightarrow H^{1}\left(X, \mathcal{O}_{\mathfrak{a}}\right) \rightarrow H^{1}\left(X, \mathcal{O}_{\mathfrak{a}}^{*}\right) \stackrel{\delta}{\rightarrow} H^{2}\left(X, \mathbb{Z}_{\mathfrak{a}}\right) \rightarrow \cdots
$$

We have similar exact sequences over $Y=p^{-1}\left(Y_{0}\right)$ so that the embedding $Y \hookrightarrow X$ induces a commutative diagram of these sequences. 
Since $X_{0}$ is a Stein manifold, $H^{1}\left(X, \mathcal{O}_{\mathfrak{a}}\right)=H^{1}\left(c_{\mathfrak{a}} X, \mathcal{O}\right)=0$ by Theorem 2.4. Thus, $\delta$ is an injection. Also, since $c_{\mathfrak{a}} X$ is homotopy equivalent to $c_{\mathfrak{a}} Y$, by the homotopy invariance for cohomology of locally constant sheaves (see, e.g., Chapter II.11 of [4]),

$$
H^{k}\left(Y, \mathbb{Z}_{\mathfrak{a}}\right)=H^{k}\left(c_{\mathfrak{a}} Y, \mathbb{Z}\right) \cong H^{k}\left(c_{\mathfrak{a}} X, \mathbb{Z}\right)=H^{k}\left(X, \mathbb{Z}_{\mathfrak{a}}\right), \quad k \geq 0
$$

(see definitions of the corresponding cohomology groups in the beginning of Section 10).

Let $c_{L} \in H^{1}\left(X, \mathcal{O}_{\mathfrak{a}}^{*}\right)$ be the cohomology class determined by the line a-bundle $L=L_{E}$ of the a-divisor $E$. We show that $\delta\left(c_{L}\right)=0$; since $\delta$ is an injection, this would imply that $L$ is isomorphic to the trivial line $\mathfrak{a}$-bundle, and hence $E$ is $\mathfrak{a}$-equivalent to an $\mathfrak{a}$-principal divisor.

Indeed, the restriction $\left.\delta\left(c_{L}\right)\right|_{Y} \in H^{2}\left(Y, \mathbb{Z}_{\mathfrak{a}}\right)$ of $\delta\left(c_{L}\right)$ to $Y$ is, by definition, the Chern class of the restriction $\left.L\right|_{Y}$. Since $\left.E\right|_{Y}$ is a-equivalent to an a-principal divisor on $Y$, the line a-bundle $\left.L\right|_{Y}$ is isomorphic to the trivial line $\mathfrak{a}$-bundle in $\mathcal{L}_{\mathfrak{a}}(Y)$, so we have $\left.\delta\left(c_{L}\right)\right|_{Y}=0$. Since the restriction homomorphism $H^{2}\left(X, \mathbb{Z}_{\mathfrak{a}}\right) \rightarrow$ $H^{2}\left(Y, \mathbb{Z}_{\mathfrak{a}}\right)$ is an isomorphism (see above), $\delta\left(c_{L}\right)=0$, as required.

Let us prove the second assertion of the theorem. Assume that $\mathfrak{a}$ is such that $\hat{G}_{\mathfrak{a}}$ is a compact topological group and $j(G) \subset \hat{G}_{\mathfrak{a}}$ is a dense subgroup, and $\operatorname{supp}(E) \cap Y=\emptyset$. We retain notation and results of parts (1) and (2) of the proof of Theorem 2.17. By definition divisor $E$ determines a holomorphic line bundle $\hat{L}_{E}$ on $c_{\mathfrak{a}} X$ and a holomorphic section $s$ of $\hat{L}_{E}$ such that $s$ is not identically zero on each 'slice' $\iota_{H}\left(X_{H}\right) \subset c_{\mathfrak{a}} X$ and $\iota^{*} \hat{L}_{E}=L_{E}$. If $\operatorname{supp}(s) \subset c_{\mathfrak{a}} X$ is zero loci of $s$, then $\iota^{-1}(\operatorname{supp}(s))=\operatorname{supp}(E)$. Let us show that $\operatorname{supp}(s) \cap c_{\mathfrak{a}} Y=\emptyset$. Indeed, assuming the contrary we find a point $x \in \iota_{H}\left(X_{H}\right) \cap c_{\mathfrak{a}} Y$ for some $H \in \Upsilon$ such that $s(x)=0$. Since $c_{\mathfrak{a}} Y \subset c_{\mathfrak{a}} X$ is open, there exists an open neighbourhood of $x$ which is contained in $c_{\mathfrak{a}} Y$. Without loss of generality we may identify this neighbourhood with $V_{0} \times K$, where $V_{0} \subset Y_{0}$ is an open coordinate chart and $K \subset \hat{G}_{\mathfrak{a}}$ is open, $K \in \mathfrak{Q}$ (see Subsection 5.1). Then $s(z, \eta)=0$ for some $(z, \eta) \in V_{0} \times K$. Let $S \subset K$ be a dense subset such that $j^{-1}(S) \subset G$, the deck transformation group of $X$ (see Subsection 2.1 for notation). By definition, $\iota^{-1}\left(V_{0} \times S\right) \subset Y$. Also, $s(\cdot, \xi) \in \mathcal{O}\left(V_{0}\right)$ for all $\xi \in K$ and $s(\cdot, \eta)$ is not identically zero. Since $s \in C\left(V_{0} \times K\right)$, by the Montel theorem there exists a sequence $\left\{s\left(\cdot, \xi_{j}\right)\right\}_{j \in \mathbb{N}},\left\{\xi_{j}\right\}_{j \in \mathbb{N}} \subset S$, converging to $s(\cdot, \eta)$ uniformly on compact subsets of $V_{0}$. Then according to the Hurwitz theorem (on zeros of a sequence of univariate holomorphic functions uniformly converging to a nonidentically zero holomorphic function), there exists $\left(w, \xi_{i}\right) \in V_{0} \times S$ such that $s\left(w, \xi_{i}\right)=0$. This implies that $\iota^{-1}\left(\left(w, \xi_{i}\right)\right) \in \operatorname{supp}(E) \cap Y$, a contradiction proving the required claim.

Thus we obtain that $\left.s\right|_{c_{\mathrm{a}} Y}$ is nowhere zero, i.e., $\left.\hat{L}_{E}\right|_{c_{\mathrm{a}} Y}$ is holomorphically trivial. In turn, $\iota^{*}\left(\left.\hat{L}_{E}\right|_{c_{\mathfrak{a}} Y}\right):=\left.\left(L_{E}\right)\right|_{Y}=L_{\left.E\right|_{Y}}$ is the trivial a-bundle on $Y$. Hence, the restriction of $E$ to $Y$ is $\mathfrak{a}$-equivalent to an $\mathfrak{a}$-principal divisor. The first part of the theorem then implies that $E$ is a-equivalent to an a-principal divisor on $X$.

The proof of the theorem is complete. 


\section{Proofs of Theorem 2.21 and Proposition 2.23}

Proof of Theorem 2.21. We will use notation and results of Subsection 5.1 and Example 4.4 in [15].

Using the axiom of choice we construct a (not necessarily continuous) right inverse $\lambda: \hat{G}_{\mathfrak{a}} \rightarrow \hat{G}_{\ell_{\infty}}$ to $\kappa$, i.e., $\kappa \circ \lambda=$ Id. Given a subset $K \subset G$, by $\hat{K}_{\mathfrak{a}} \subset \hat{G}_{\mathfrak{a}}$ and $\hat{K}_{\ell_{\infty}} \subset \hat{G}_{\ell_{\infty}}$ we denote the closures of sets $j_{\mathfrak{a}}(K)$ and $j_{\ell_{\infty}}(K)$ in $\hat{G}_{\mathfrak{a}}$ and $\hat{G}_{\ell_{\infty}}$, respectively.

For $\Pi\left(U_{0}, K\right):=\Pi_{\gamma_{*}}\left(U_{0}, K\right)$ we have a commutative diagram

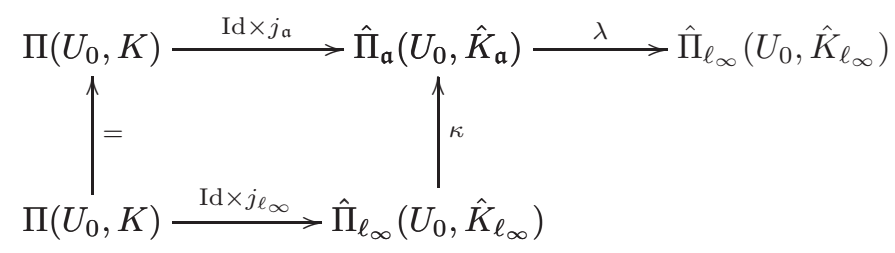

All maps, except possibly for $\lambda$, are continuous.

We will need the following results.

Lemma 11.1. Under the hypotheses of the theorem, there exists a unique function $\hat{f} \in \mathcal{O}\left(\hat{\Pi}_{\mathfrak{a}}\left(U_{0}, \hat{K}_{\mathfrak{a}}\right)\right)$ such that

$$
\left.f\right|_{\Pi\left(U_{0}, K\right)}=\left(\operatorname{Id} \times j_{\mathfrak{a}}\right)^{*} \hat{f} .
$$

Proof. Since $f \in \mathcal{O}_{\ell_{\infty}}(X)$, there exists a function $\tilde{f} \in \mathcal{O}\left(\hat{\Pi}_{\ell_{\infty}}\left(U_{0}, \hat{K}_{\mathfrak{a}}\right)\right)$ such that $\left.f\right|_{\Pi\left(U_{0}, K\right)}=\left(\operatorname{Id} \times j_{\ell_{\infty}}\right)^{*} \tilde{f}$. We set $\hat{f}:=(\operatorname{Id} \times \lambda)^{*} \tilde{f}: \hat{\Pi}_{\mathfrak{a}}\left(U_{0}, \hat{K}_{\mathfrak{a}}\right) \rightarrow \mathbb{C}$. Clearly, $(11.2)$ is satisfied. Identifying $\hat{\Pi}_{\mathfrak{a}}\left(U_{0}, \hat{K}_{\mathfrak{a}}\right)$ with $U_{0} \times \hat{K}_{\mathfrak{a}}$ (see $(5.2)$ ), we obtain that $\hat{f}(\cdot, \omega) \in \mathcal{O}\left(U_{0}\right)$ for all $\omega \in \hat{K}_{\mathfrak{a}}$. It remains to show that $\hat{f}$ is continuous. Since $f \in C_{\mathfrak{a}}(Z)$, there exists a function $F \in C\left(\hat{\Pi}_{\mathfrak{a}}\left(Z_{0}, \hat{K}_{\mathfrak{a}}\right)\right)$ such that $\left.f\right|_{\Pi\left(Z_{0}, K\right)}=$ $\left(\operatorname{Id} \times j_{\mathfrak{a}}\right)^{*} F$. Also, since $\left(\operatorname{Id} \times j_{\mathfrak{a}}\right)\left(\Pi\left(Z_{0}, K\right)\right)$ is dense in $\hat{\Pi}_{\mathfrak{a}}\left(Z_{0}, \hat{K}_{\mathfrak{a}}\right)$ and diagram (11.1) is commutative,

$$
\left.\hat{f}\right|_{\hat{\Pi}_{\mathfrak{a}}\left(Z_{0}, \hat{K}_{\mathfrak{a}}\right)}=F
$$

We identify $\hat{\Pi}_{\mathfrak{a}}\left(U_{0}, \hat{K}_{\mathfrak{a}}\right)$ with $U_{0} \times \hat{K}_{\mathfrak{a}}$, and $\hat{\Pi}_{\mathfrak{a}}\left(Z_{0}, \hat{K}_{\mathfrak{a}}\right)$ with $Z_{0} \times \hat{K}_{\mathfrak{a}}$. Suppose that $\hat{f}$ is discontinuous, i.e., there exists a net $\left\{\left(z_{\alpha}, \omega_{\alpha}\right)\right\} \subset U_{0} \times \hat{K}_{\mathfrak{a}},\left(z_{\alpha}, \omega_{\alpha}\right) \rightarrow(z, \omega) \in$ $U_{0} \times \hat{K}_{\mathfrak{a}}$, such that $\lim _{\alpha} \hat{f}\left(z_{\alpha}, \omega_{\alpha}\right)$ exists but does not coincide with $\hat{f}(z, \omega)$. Using the Montel theorem we find a subnet $\left\{\hat{f}\left(\cdot, \omega_{\alpha_{\beta}}\right)\right\}$ of the net $\left\{\hat{f}\left(\cdot, \omega_{\alpha}\right)\right\} \subset \mathcal{O}\left(U_{0}\right)$ which converges to a function $g$. Since $\left.\hat{f}\right|_{Z_{0} \times \hat{K}_{\mathfrak{a}}}$ is continuous and $Z_{0}$ is a uniqueness set for functions in $\mathcal{O}\left(U_{0}\right), g=\hat{f}(\cdot, \omega)$. But $g(z)=\lim _{\alpha} \hat{f}\left(z_{\alpha}, \omega_{\alpha}\right)$, a contradiction showing that $\hat{f} \in C\left(\hat{\Pi}_{\mathfrak{a}}\left(Z_{0}, \hat{K}_{\mathfrak{a}}\right)\right)$. Hence, $\hat{f} \in \mathcal{O}\left(\hat{\Pi}_{\mathfrak{a}}\left(Z_{0}, \hat{K}_{\mathfrak{a}}\right)\right)$.

Lemma 11.2. We have $\cup_{i=1}^{m} \hat{L}_{\mathfrak{a}} \cdot j_{\mathfrak{a}}\left(g_{i}\right)=\hat{G}_{\mathfrak{a}}$.

(Recall that $\hat{L}_{\mathfrak{a}}$ is the closure of $j_{\mathfrak{a}}(L)$ in $\hat{G}_{\mathfrak{a}} \cdot$ ) 
Proof. Indeed, $\cup_{i=1}^{m} \hat{L}_{\mathfrak{a}} \cdot j_{\mathfrak{a}}\left(g_{i}\right)$ is a closed subset of $\hat{G}_{\mathfrak{a}}$ containing $j_{\mathfrak{a}}(G)$, as by the assumption of the theorem $\cup_{i=1}^{m} L \cdot g_{i}=G$. Since set $j_{\mathfrak{a}}(G)$ is dense in $\hat{G}_{\mathfrak{a}}$, we obtain that $\cup_{i=1}^{m} \hat{L}_{\mathfrak{a}} \cdot j_{\mathfrak{a}}\left(g_{i}\right)=\hat{G}_{\mathfrak{a}}$.

We now complete the proof of Theorem 2.21 by means of an analytic continuation-type argument.

Let us consider the open cover $\mathcal{U}=\left\{U_{0, \gamma}\right\}$ of $X_{0}$ and the corresponding system of trivializations $\psi_{\gamma}: p^{-1}\left(U_{0, \gamma} \times G\right) \rightarrow U_{0, \gamma} \times G$ of the covering $p: X_{0} \rightarrow X$ introduced in Subsection 2.4. (Recall that $\Pi_{\gamma}\left(U_{0, \gamma}, S\right):=\psi_{\gamma}^{-1}\left(U_{0} \times S\right), S \subset G$.) This system determines a system of trivializations $\bar{\psi}_{\gamma}: \hat{\Pi}_{\mathfrak{a}, \gamma}\left(U_{0, \gamma}, L\right) \rightarrow U_{0, \gamma} \times L$, $L \subset \hat{G}_{\mathfrak{a}}$, of the fibrewise compactification $\bar{p}: c_{\mathfrak{a}} X \rightarrow X_{0}$, see Subsection 5.1. Passing to a refinement of $\mathcal{U}$, if necessary, we may and will assume without loss of generality that all nonempty sets $U_{0, \gamma} \cap U_{0, \delta}$ are connected and simply connected, and that $U_{0}=U_{0, \gamma_{*}}$ for $\gamma_{*}$ from the statement of the theorem.

First, we will prove that

$\left.{ }^{*}\right)$ there exists a function $\hat{f}_{U_{0}} \in \mathcal{O}\left(\bar{p}^{-1}\left(U_{0}\right)\right)$ such that $\iota^{*} \hat{f}_{U_{0}}=\left.f\right|_{p^{-1}\left(U_{0}\right)}$.

Let us fix $1 \leq i \leq m$.

Lemma 11.3. There exist families $\left\{U_{0, \gamma_{l}}\right\}_{l=1}^{s(i)} \subset \mathcal{U}$ and $\left\{K_{l}\right\}_{l=1}^{s(i)} \subset G$ such that

(1) $\gamma_{1}=\gamma_{s(i)}=\gamma_{*}, K_{1}:=K$ and $K_{s(i)}=K \cdot g_{i}$,

(2) $U_{0, \gamma_{l}} \cap U_{0, \gamma_{l+1}} \neq \varnothing$ for all $1 \leq l \leq s(i)-1$, and

(3) $\Pi_{\gamma_{l}}\left(U_{0, \gamma_{l}} \cap U_{0, \gamma_{l+1}}, K_{l}\right)=\Pi_{\gamma_{l+1}}\left(U_{0, \gamma_{l}} \cap U_{0, \gamma_{l+1}}, K_{l+1}\right)$ for all $1 \leq l \leq s(i)-1$.

Proof. Take $x_{0} \in U_{0}$ and define $y_{0}:=\psi_{\gamma_{*}}^{-1}\left(x_{0}, 1\right)$. Since covering $p: X \rightarrow X_{0}$ is regular, there exists a continuous path joining $y_{0}$ and $g_{i} \cdot y_{0}$ obtained as the lift of a loop $\gamma_{0}:[0,1] \rightarrow X_{0}$ with basepoint $x_{0}$. Then there exist a partition $0=t_{0}<t_{1}<\cdots<t_{s(i)}=1$ of $[0,1]$ and a family $\left\{U_{0, \gamma_{l}}\right\}_{l=1}^{s(i)} \subset \mathcal{U}$ such that

$$
\begin{aligned}
& \gamma_{0}([0,1]) \subset \bigcup_{i=1}^{s(i)} U_{0, \gamma_{i}} ; \quad U_{0, \gamma_{1}}=U_{0, \gamma_{s(i)}}:=U_{0}\left(=U_{0, \gamma_{*}}\right) ; \\
& \gamma_{0}\left(\left[t_{i}, t_{i+1}\right]\right) \subset U_{0, \gamma_{i+1}} \quad \forall i \geq 0 .
\end{aligned}
$$

Now, we define $K_{1}=K$ and $K_{l+1}:=K_{l} \cdot c_{\gamma_{l} \gamma_{l+1}}$ for all $1 \leq l \leq s(i)-1$ (note that $U_{0, \gamma_{l}} \cap U_{0, \gamma_{l+1}} \neq \varnothing$ by our construction), where $\left\{c_{\delta \gamma}\right\}$ is the 1-cocycle on $\mathcal{U}$ determining covering $p: X \rightarrow X_{0}$ (see Subsection 2.4). Clearly, conditions (1)-(3) are satisfied.

Further, using Lemma 11.1 we can find a function $\hat{f}_{1} \in \mathcal{O}\left(\hat{\Pi}_{\mathfrak{a}, \gamma_{1}}\left(U_{0, \gamma_{1}}, \hat{K}_{1 \mathfrak{a}}\right)\right)$ such that $\iota^{*} \hat{f}_{1}=f$ on $\Pi_{\gamma_{1}}\left(U_{0, \gamma_{1}}, K_{1}\right)$. Since the open set $U_{0, \gamma_{1}} \cap U_{0, \gamma_{2}}(\neq \varnothing)$ is a uniqueness set for functions in $\mathcal{O}\left(U_{0, \gamma_{2}}\right)$, we can apply Lemma 11.1 to $\left.f\right|_{\Pi_{\gamma_{2}}\left(U_{0, \gamma_{2}}, K_{2}\right)}$ to find a function $\hat{f}_{2} \in \mathcal{O}\left(\hat{\Pi}_{\mathfrak{a}, \gamma_{2}}\left(U_{0, \gamma_{2}}, \hat{K}_{2 \mathfrak{a}}\right)\right)$ such that $\iota^{*} \hat{f}_{2}=f$ on $\Pi_{\gamma_{2}}\left(U_{0, \gamma_{2}}, K_{2}\right)$. (Indeed, as the set $Z$ in the lemma we can take $\Pi_{\gamma_{2}}\left(V, K_{2}\right)$, where $V$ is a compact subset of $U_{0, \gamma_{1}} \cap U_{0, \gamma_{2}}$ with nonempty interior. Then $f=\iota^{*} \hat{f}_{1}$ on $Z$ and the continuous function $\hat{f}_{1}$ defined on compact subset $\hat{\Pi}_{\mathfrak{a}, \gamma_{1}}\left(U_{0, \gamma_{1}}, \hat{K}_{1 \mathfrak{a}}\right)$ of $c_{\mathfrak{a}} X$ admits a continuous extension to $c_{\mathfrak{a}} X$ by the Tietze-Urysohn theorem. Thus, $f \in C_{\mathfrak{a}}(Z)$, 
as required in the lemma.) We repeat this construction for $3 \leq l \leq s(i)$ to obtain functions $\hat{f}_{l} \in \mathcal{O}\left(\hat{\Pi}_{\mathfrak{a}, \gamma_{l}}\left(U_{0, \gamma_{l}}, \hat{K}_{l \mathfrak{a}}\right)\right)$ such that $\iota^{*} \hat{f}_{l}=f$ on $\Pi_{\gamma_{l}}\left(U_{0, \gamma_{l}}, K_{l}\right)$. Using these arguments for all $1 \leq i \leq m$ we obtain functions $\hat{f}_{s(i)} \in \mathcal{O}\left(\hat{\Pi}_{\mathfrak{a}, \gamma_{*}}\left(U_{0, \gamma_{*}}, \hat{K}_{\mathfrak{a}}\right.\right.$. $\left.\left.j_{\mathfrak{a}}\left(g_{i}\right)\right)\right)$ such that $\iota^{*} \hat{f}_{s(i)}=\left.f\right|_{\Pi_{\gamma_{*}}\left(U_{\gamma_{*}}, K \cdot g_{i}\right)}$.

Let $K_{\mathfrak{a}}^{\circ}$ denote the interior of $\hat{K}_{\mathfrak{a}}$. Then $K_{\mathfrak{a}}^{\circ} \cdot j_{\mathfrak{a}}\left(g_{i}\right)$ is the interior of $\hat{K}_{\mathfrak{a}} \cdot j_{\mathfrak{a}}\left(g_{i}\right)$ for all $i \geq 1$. We have $\hat{f}_{s(i)}=\hat{f}_{s(j)}$ on $\hat{\Pi}_{\mathfrak{a}, \gamma_{*}}\left(U_{0, \gamma_{*}}, K_{\mathfrak{a}}^{\circ} \cdot j_{\mathfrak{a}}\left(g_{i}\right)\right) \cap \hat{\Pi}_{\mathfrak{a}, \gamma_{*}}\left(U_{0, \gamma_{*}}, K_{\mathfrak{a}}^{\circ}\right.$. $\left.j_{\mathfrak{a}}\left(g_{j}\right)\right) \neq \emptyset$ since by our construction these functions are continuous and coincide on dense subset $\iota\left(\Pi_{\gamma_{*}}\left(U_{0, \gamma_{*}}, K \cdot g_{i}\right) \cap \Pi_{\gamma_{*}}\left(U_{0, \gamma_{*}}, K \cdot g_{j}\right)\right)$ of the latter set. Finally, by Lemma 11.2, $\cup_{i=1}^{m} \hat{L}_{\mathfrak{a}} \cdot j_{\mathfrak{a}}\left(g_{i}\right)=\hat{G}_{\mathfrak{a}}$, and since $\hat{L}_{\mathfrak{a}} \subset K_{\mathfrak{a}}^{\circ}$ by the assumption of the theorem, we have $\cup_{i=1}^{m} K_{\mathfrak{a}}^{\circ} \cdot j_{\mathfrak{a}}\left(g_{i}\right)=\hat{G}_{\mathfrak{a}}$. This shows that $\bar{p}^{-1}\left(U_{0}\right)=$ $\cup_{i=1}^{m} \hat{\Pi}_{\mathfrak{a}, \gamma_{*}}\left(U_{0, \gamma_{*}}, K_{\mathfrak{a}}^{\circ} \cdot j_{\mathfrak{a}}\left(g_{i}\right)\right)$. Therefore $\left.\hat{f}_{U_{0}}\right|_{\hat{\Pi}_{\mathfrak{a}, \gamma_{*}}\left(U_{0, \gamma_{*}}, K_{\mathfrak{a}}^{\circ} \cdot j_{\mathfrak{a}}\left(g_{i}\right)\right)}:=\hat{f}_{s(i)}, 1 \leq i \leq$ $m$, is a function in $\mathcal{O}\left(\bar{p}^{-1}\left(U_{0}\right)\right)$ satisfying $(*)$ as required. By definition (cf. (2.3)), $\left.f\right|_{p^{-1}\left(U_{0}\right)} \in \mathcal{O}_{\mathfrak{a}}\left(p^{-1}\left(U_{0}\right)\right)$.

Let $W_{0} \subset X_{0}$ be the maximal connected open subset which consists of unions of elements of the cover $\mathcal{U}$ and such that $\left.f\right|_{p^{-1}\left(W_{0}\right)} \in \mathcal{O}_{\mathfrak{a}}\left(p^{-1}\left(W_{0}\right)\right.$ ). (Existence of $W_{0}$ follows from Zorn's lemma; also, $U_{0} \subset W_{0}$.) Let us show that $W_{0}=X_{0}$. Assuming the contrary, we find (because of connectedness of $X_{0}$ ) a subset $U_{0}^{\prime} \in \mathcal{U}$ such that $W_{0} \cap U_{0}^{\prime} \neq \emptyset$ and $W_{0}$ is a proper subset of the open connected set $W_{0} \cup U_{0}^{\prime}$. Now in conditions of Theorem 2.21 we replace $U_{0}, Z$ and $K$ by sets $U_{0}^{\prime}, Z^{\prime}:=p^{-1}\left(Z_{0}^{\prime}\right)$, where $Z_{0}^{\prime} \Subset U_{0}^{\prime} \cap U_{0}$ is compact with nonempty interior (hence, a uniqueness set for functions in $\left.\mathcal{O}\left(U_{0}^{\prime}\right)\right)$, and $K^{\prime}:=G$, respectively. Since $\left.f\right|_{p^{-1}\left(U_{0}\right)} \in \mathcal{O}_{\mathfrak{a}}\left(p^{-1}\left(U_{0}\right)\right)$, we have $\left.f\right|_{Z^{\prime}} \in C_{\mathfrak{a}}\left(Z^{\prime}\right)$. Therefore claim $\left(^{*}\right)$ in this setting gives a function $\hat{f}_{U_{0}^{\prime}} \in$ $\mathcal{O}\left(\bar{p}^{-1}\left(U_{0}^{\prime}\right)\right)$ such that $\iota^{*} \hat{f}_{U_{0}^{\prime}}=\left.f\right|_{p^{-1}\left(U_{0}^{\prime}\right)}$, i.e., $\left.f\right|_{p^{-1}\left(U_{0}^{\prime}\right)} \in \mathcal{O}_{\mathfrak{a}}\left(p^{-1}\left(U_{0}^{\prime}\right)\right)$. Since $\left.f\right|_{p^{-1}\left(W_{0}\right)} \in \mathcal{O}_{\mathfrak{a}}\left(p^{-1}\left(W_{0}\right)\right)$, this implies that $\left.f\right|_{p^{-1}\left(W_{0} \cup U_{0}^{\prime}\right)} \in \mathcal{O}_{\mathfrak{a}}\left(p^{-1}\left(W_{0} \cup U_{0}^{\prime}\right)\right)$ contradicting the maximality of $W_{0}$. Thus, $W_{0}=X_{0}$ and $f \in \mathcal{O}_{\mathfrak{a}}(X)$.

The proof of the theorem is complete.

Proof of Proposition 2.23. (a) $\Rightarrow$ (b). Suppose that there exist $g_{1}, \ldots, g_{m} \in G$ such that $\cup_{i=1}^{m} K \cdot g_{i}=G$. Let us show that the closure $\hat{K}_{\mathfrak{a}}$ of $j(K), j:=j_{\mathfrak{a}}$, in $\hat{G}_{\mathfrak{a}}$ has a nonempty interior $\hat{K}_{\mathfrak{a}}^{\circ}$. Indeed, by Lemma $11.2, \cup_{i=1}^{m} \hat{K}_{\mathfrak{a}} \cdot j\left(g_{i}\right)=\hat{G}_{\mathfrak{a}}$. Assuming that $\hat{K}_{\mathfrak{a}} \neq \hat{G}_{\mathfrak{a}}$ (in this case the statement is trivial) we may choose $1 \leq k \leq m-1$ such that $K^{\prime}=\cup_{i=1}^{k} \hat{K}_{\mathfrak{a}} \cdot j\left(g_{i}\right)$ does not cover $\hat{G}_{\mathfrak{a}}$ but $\cup_{i=1}^{k+1} \hat{K}_{\mathfrak{a}} \cdot j\left(g_{i}\right)=\hat{G}_{\mathfrak{a}}$. Thus the complement of $K^{\prime}$ is a nonempty open subset of $\hat{K}_{\mathfrak{a}} \cdot j\left(g_{k+1}\right)$. This implies that $\hat{K}_{\mathfrak{a}}^{\circ} \neq \emptyset$.

(b) $\Rightarrow(\mathrm{c})$. Let $U \subset \hat{G}_{\mathfrak{a}}$ be open. Since $j(G)$ is a dense subgroup of the compact topological group $\hat{G}_{\mathfrak{a}}$, the set $\cup_{g \in G} U \cdot j(g)$ coincides with $\hat{G}_{\mathfrak{a}}$. (For otherwise, there exists $v \in \hat{G}_{\mathfrak{a}}$ such that the closure in $\hat{G}_{\mathfrak{a}}$ of the set $\{v \cdot j(g)\}_{g \in G}$ is a proper subset of $\hat{G}_{\mathfrak{a}}$ which contradicts the density of $j(G)$ in $\hat{G}_{\mathfrak{a}}$.) Thus there exist $g_{1}, \ldots, g_{m} \in G$ such that $\cup_{i=1}^{m} U \cdot j\left(g_{i}\right)=\hat{G}_{\mathfrak{a}}$. This implies that $\cup_{i=1}^{m} j^{-1}(U) \cdot g_{i}=G$.

Now, suppose that $K \subset G$ is such that $\hat{K}_{\mathfrak{a}}^{\circ} \neq \emptyset$. Choose an open set $U \Subset \hat{K}_{\mathfrak{a}}^{\circ}$ and define $L:=j^{-1}(U) \subset G$. The previous argument shows that the pair $L \subset K$ satisfies conditions of Theorem 2.21.

$(\mathrm{c}) \Rightarrow(\mathrm{a})$. Follows from the definitions. 


\section{Proofs of Proposition 2.24 and Theorems 2.26, 2.28 and 2.29}

\subsection{Proof of Proposition 2.24}

It is easy to see that any function $f \in \mathcal{O}_{\mathfrak{a}}(X)$ is locally Lipschitz with respect to metric $d$ (see Section 1), i.e.,

$$
\left|f\left(x_{1}, g\right)-f\left(x_{2}, g\right)\right| \leq C d\left(\left(x_{1}, g\right),\left(x_{2}, g\right)\right)=C d_{0}\left(x_{1}, x_{2}\right)
$$

for all $\left(x_{1}, g\right),\left(x_{2}, g\right) \in W_{0} \times G \cong p^{-1}\left(W_{0}\right)$, where $W_{0} \Subset X_{0}$ is a simply connected coordinate chart. (Here $C$ depends on $d_{0}$ and $W_{0}$ only.) We set $f_{x_{0}}:=\left.f\right|_{p^{-1}\left(x_{0}\right)} \in$ $\mathfrak{a}, x_{0} \in X_{0}$, and define

$$
\tilde{f}\left(x_{0}\right):=f_{x_{0}}, \quad x_{0} \in X_{0} .
$$

Then $\tilde{f}$ is a section of bundle $C_{\mathfrak{a}} X_{0}$. Using (12.1) for any linear functional $\varphi \in \mathfrak{a}^{*}$ we have $\varphi(\tilde{f}(x)(g)):=\varphi(f(x, g)) \in \mathcal{O}\left(W_{0}\right), g \in G, x \in W_{0} \Subset X_{0}$, a simply connected coordinate chart, see [44] or [8] for similar arguments. Thus $\tilde{f}$ is a holomorphic section of $C_{\mathfrak{a}} X_{0}$. Reversing these arguments we obtain that any holomorphic section of $C_{\mathfrak{a}} X_{0}$ determines a holomorphic a-function on $X$.

\subsection{Proof of Theorem 2.26}

Let $B$ be a (complex) Banach space. We define

$$
\mathcal{A}\left(D_{0}, B\right):=C\left(\bar{D}_{0}, B\right) \cap \mathcal{O}\left(D_{0}, B\right) .
$$

Consider a family of bounded linear operators $\mathcal{L}_{z}^{B}: B \rightarrow \mathcal{A}\left(D_{0}, B\right), z \in D_{0}$, holomorphic in $z$ such that $\mathcal{L}_{z}^{B}(b)=b$ for every $b \in B$ and $\sup _{z \in D_{0}}\left\|\mathcal{L}_{z}^{B}\right\|=1$ defined by the formula

$$
\mathcal{L}_{z}^{B}(b)(x):=b \quad \text { for all } x \in D_{0} .
$$

We use notation and results of Subsection 2.5.1. Namely, we identify functions in algebra $\mathfrak{a}_{z}, z \in D_{0}$, with sections over $z$ of the holomorphic Banach vector bundle $\tilde{p}: C_{\mathfrak{a}} X_{0} \rightarrow X_{0}$ associated to the principal fibre bundle $p: X \rightarrow X_{0}$ and having fibre $\mathfrak{a}$, and functions in $\mathcal{O}_{\mathfrak{a}}(D)$ with holomorphic sections of $\left.\mathcal{O}\left(C_{\mathfrak{a}} X_{0}\right)\right|_{D_{0}}$. Recall that there is a holomorphic Banach vector bundle $E$ such that $C_{\mathfrak{a}} X_{0} \oplus E=X_{0} \times B$ for some Banach space $B$. By $q: X_{0} \times B \rightarrow C_{\mathfrak{a}} X_{0}$ and $i: C_{\mathfrak{a}} X_{0} \rightarrow X_{0} \times B, q \circ i=\mathrm{Id}$, we denote the corresponding bundle morphisms. Now, for every $h \in \mathfrak{a}_{z}$ we define

$$
L_{z}(h):=\left(q \circ \mathcal{L}_{z}^{B} \circ i\right)(h) \in \mathcal{A}_{\mathfrak{a}}(D) .
$$

Clearly, the family $\left\{L_{z}\right\}_{z \in D_{0}}$ satisfies conditions (1), (2) of Theorem 2.26.

\subsection{Proof of Theorem 2.28}

The arguments below are analogous to those in [7]. 
Using the construction of Subsection 2.5.1 we identify functions in $C_{\mathfrak{a}}(X)$ and $\mathcal{O}_{\mathfrak{a}}(X)$ with continuous and holomorphic sections of the holomorphic Banach vector bundle $\tilde{p}: C_{\mathfrak{a}} X_{0} \rightarrow X_{0}$ associated to the principal fibre bundle $p: X \rightarrow X_{0}$ and having fibre a. Further, there exist holomorphic Banach vector bundles $p_{1}: E_{1} \rightarrow X_{0}$ and $p_{2}: E_{2} \rightarrow X_{0}$ with fibres $B_{1}$ and $B_{2}$ such that $E_{2}=E_{1} \oplus C_{\mathfrak{a}} X_{0}$ and $E_{2}$ is holomorphically trivial, i.e., $E_{2} \cong X_{0} \times B_{2}$ (see, e.g., [55]); so continuous and holomorphic sections of $E_{2}$ can be identified with $B_{2}$-valued continuous and holomorphic functions on $X_{0}$. By $q: E_{2} \rightarrow C_{\mathfrak{a}} X_{0}$ and $i: C_{\mathfrak{a}} X_{0} \rightarrow E_{2}$ we denote the corresponding quotient and embedding homomorphisms of the bundles so that $q \circ i=\mathrm{Id}$.

As before we identify function $f$ satisfying the hypothesis of Theorem 2.28 with a continuous section of $C_{\mathfrak{a}} X_{0}$ over $\partial D_{0}$. Then $h:=i(f) \in C\left(\partial D_{0}, B_{2}\right)$. Since $f \in C_{\mathfrak{a}}(\partial D)$ satisfies the tangential Cauchy-Riemann equations, $h$ satisfies the weak tangential Cauchy-Riemann equations on $\partial D_{0}$ :

$$
\int_{\partial D_{0}}(\varphi \circ h) \bar{\partial} \omega=0
$$

for any smooth form $\omega \in \Lambda^{n, n-2}\left(X_{0}\right)$ having compact support and any $\varphi \in B_{2}^{*}$. Hence, applying the Hartogs-type theorem of [36] to functions $\varphi \circ h$ we obtain that there exists a function $H \in \mathcal{O}\left(D_{0}, B_{2}^{* *}\right) \cap C\left(\bar{D}_{0}, B_{2}^{* *}\right)$, where the second dual $B_{2}^{* *}$ of $B_{2}$ is considered with weak* topology, such that $\left.H\right|_{\partial D_{0}}=h$ (here $B_{2}$ is naturally identified with its isometric copy in $\left.B_{2}^{* *}\right)$.

Now, we use the integral representation result of Corollary 5.4 in [28], asserting that there exist a compact subset $S \subset \bar{D}_{0} \backslash D_{0}$, a positive Radon measure $\mu$ on $S$ and a function $Q$ on $D_{0} \times S$ such that (a) $Q(\cdot, y)$ is holomorphic for all $y \in S$; (b) $Q(x, \cdot)$ is $\mu$-integrable for all $x \in D_{0}$; (c) $x \mapsto \int_{S}|Q(x, y)| d \mu(y)$ is continuous; (d) for any function $w \in \mathcal{O}\left(D_{0}\right) \cap C\left(\bar{D}_{0}\right)$

$$
w(x)=\int_{S} Q(x, y) f(y) d \mu(y) \quad \text { for all } x \in D_{0} .
$$

Using the Bochner integration we define

$$
H^{\prime}(x):=\int_{M} Q(x, y) h(y) d \mu(y), \quad x \in D_{0} .
$$

Then $H^{\prime} \in C\left(D_{0}, B_{2}\right)$. Since the Bochner integral commutes with the action of bounded linear functionals, $\varphi \circ H^{\prime}=\varphi \circ H$ on $D_{0}$ for all $\varphi \in B_{2}^{*}$. Thus, $H^{\prime}=H$ on $D_{0}$ and so $H \in \mathcal{O}\left(D_{0},\left(B_{2}, w\right)\right) \cap C\left(\bar{D}_{0},\left(B_{2}, w\right)\right)$, where $\left(B_{2}, w\right)$ is $B_{2}$ equipped with weak topology, and $H \in \mathcal{O}\left(D_{0}, B_{2}\right)$.

Now, the required holomorphic extension of $f$ is given by $F:=q\left(H^{\prime}\right)$. Indeed, by our construction $\left.F\right|_{D_{0}} \in \mathcal{O}\left(D_{0}, C_{\mathfrak{a}} X_{0}\right)$. By Proposition $2.24,\left.F\right|_{D_{0}}$ can be viewed as a function in $\mathcal{O}_{\mathfrak{a}}(D)$. Further, since map $q$ is continuous also if we equip fibres of the corresponding bundles with weak topologies, $F$ is a continuous section of $\left(C_{\mathfrak{a}} X_{0}, w\right)$ over $\bar{D}_{0}$, i.e., of $C_{\mathfrak{a}} X_{0}$ with fibres endowed with weak topology. Using presentation (2.9) of $C_{\mathfrak{a}} X_{0}$ and evaluation functionals at points of $G$, we easily 
obtain from the weak continuity of $F$ that considered as a function on $X$ it is continuous up to the boundary. Hence, $F \in \mathcal{O}_{\mathfrak{a}}(D) \cap C(\bar{D})$ and $\left.F\right|_{\partial D}=f$, as required.

\subsection{Proof of Theorem 2.29}

Let $D_{0}$ be a relatively compact subdomain of $X_{0}, D:=p^{-1}\left(D_{0}\right)$. We set $\mathcal{A}_{\mathfrak{a}}(D):=$ $\mathcal{O}_{\mathfrak{a}}(D) \cap C_{\mathfrak{a}}(\bar{D})$. By $\mathcal{A}_{\iota}(D)$ we denote the space of holomorphic functions $f \in$ $\mathcal{A}_{\mathfrak{a}}(D)$ such that for every $x_{0} \in \bar{D}_{0}$ the function $g \mapsto f(g \cdot x)\left(g \in G, x \in p^{-1}\left(x_{0}\right)\right)$ is in $\mathfrak{a}_{\iota}$, and by $\mathcal{A}_{0}(D)$ the $\mathbb{C}$-linear hull of spaces $\mathcal{A}_{\iota}(D), \iota \in I$.

Theorem 2.29 is a corollary of the following result.

Theorem 12.1. If $X_{0}$ is a Stein manifold and $D_{0} \subset X_{0}$ is a strictly pseudoconvex domain, then $\mathcal{A}_{0}(D)$ is dense in $\mathcal{A}_{\mathfrak{a}}(D)$.

First, we deduce Theorem 2.29 from Theorem 12.1 and then prove the latter.

By $C_{\mathfrak{a}_{\iota}} X_{0}(\iota \in I)$ we denote the holomorphic Banach vector bundle associated to the principal fibre bundle $p: X \rightarrow X_{0}$ and having fibre $\mathfrak{a}_{\iota}$ (see (2.9)). For a given open subset $D_{0} \subset X_{0}$ by $\mathcal{O}\left(D_{0}, C_{\mathfrak{a}_{\iota}} X_{0}\right)$ we denote the space of holomorphic sections of bundle $C_{\mathfrak{a}_{\iota}} X_{0}$ over $D_{0}$ endowed with the topology of uniform convergence on compact subsets of $D_{0}$ which makes it a Fréchet space. We have an isomorphism of Fréchet spaces

$$
\mathcal{O}_{\mathfrak{a}_{\iota}}(D) \stackrel{\cong}{\rightrightarrows} \mathcal{O}\left(D_{0}, C_{\mathfrak{a}_{\iota}} X_{0}\right)
$$

(the proof repeats literally that of Proposition 2.24).

Let $X_{0}$ be a Stein manifold, $Y_{0} \Subset X_{0}$ be open such that $\bar{Y}_{0}$ is holomorphically convex, and $D_{0} \subset X_{0}$ be an open neighbourhood of $\bar{Y}_{0}$. We set $Y:=p^{-1}\left(Y_{0}\right)$.

Proposition 12.2. Let $f \in \mathcal{O}_{\mathfrak{a}_{\iota}}(D)$. For every $\varepsilon>0$ there exists $h \in \mathcal{O}_{\mathfrak{a}_{\iota}}(X)$ such that $\sup _{z \in Y}|f(z)-h(z)|<\varepsilon$.

Proof. We need the following approximation result established in Theorem C of $[16]$.

Let $B$ be a complex Banach space and $\mathcal{O}\left(X_{0}, B\right)$ the space of $B$-valued holomorphic functions on $X_{0}$.

$(\diamond)$ Let $\hat{f} \in \mathcal{O}\left(D_{0}, B\right)$. For every $\varepsilon>0$ there exists $\hat{h} \in \mathcal{O}\left(X_{0}, B\right)$ such that $\sup _{z \in Y_{0}}\|\hat{f}(z)-\hat{h}(z)\|_{B}<\varepsilon$.

Further, since $X_{0}$ is a Stein manifold, there exist holomorphic Banach vector bundles $p_{1}: E_{1} \rightarrow X_{0}$ and $p_{2}: E_{2} \rightarrow X_{0}$ with fibres $B_{1}$ and $B_{2}$ such that $E_{2}=E_{1} \oplus C_{\mathfrak{a}_{\iota}} X_{0}$ and $E_{2}$ is holomorphically trivial, i.e., $E_{2} \cong X_{0} \times B_{2}$ (cf. the proof of Theorem 2.28). Thus, any holomorphic section of $E_{2}$ can be naturally identified with a $B_{2}$-valued holomorphic function on $X_{0}$. By $q: E_{2} \rightarrow$ $C_{\mathfrak{a}_{\iota}} X_{0}$ and $i: C_{\mathfrak{a}_{\iota}} X_{0} \rightarrow E_{2}$ we denote the corresponding quotient and embedding homomorphisms of these bundles so that $q \circ i=$ Id. Given a function $f \in \mathcal{O}_{\mathfrak{a}_{\iota}}(D)$ by $\hat{f} \in \mathcal{O}\left(D_{0}, C_{\mathfrak{a}_{\iota}} X_{0}\right)$ we denote its image under isomorphism (12.3). 
Set $\tilde{f}:=i(\hat{f}) \in \mathcal{O}\left(D_{0}, B_{2}\right)$. By $(\diamond)$, for every $\tilde{\varepsilon}>0$ there exists a function $\tilde{h} \in \mathcal{O}\left(X_{0}, B_{2}\right)$ such that $\sup _{z \in Y_{0}}\|\tilde{f}(z)-\tilde{h}(z)\|_{B_{2}}<\tilde{\varepsilon}$. We define $\hat{h}:=q(\tilde{h}) \in$ $\mathcal{O}\left(X_{0}, C_{\mathfrak{a}_{\iota}} X_{0}\right)$ and by $h \in \mathcal{O}_{\mathfrak{a}_{\iota}}(X)$ denote the image of $\hat{h}$ under the inverse isomorphism in (12.3). The continuity of $i$ and $q$ and the compactness of $\bar{Y}_{0}$ now imply that $\sup _{z \in Y}|f(z)-h(z)|<C \tilde{\varepsilon}$ for some $C>0$ independent of $\hat{f}$ and $\tilde{\varepsilon}$.

Using this proposition we complete the proof of Theorem 2.29 as follows.

Let $f \in \mathcal{O}_{\mathfrak{a}}(X)$. It suffices to show that for a sequence $Y_{0,1} \Subset \cdots \Subset Y_{0, k} \Subset \cdots$ of open subsets of $X_{0}$ such that $\cup_{k} Y_{0, k}=X_{0}$ and for any $\varepsilon>0$ there exist functions $h_{k} \in \mathcal{O}_{0}(X)$ such that $\sup _{x \in Y_{k}}\left|f(x)-h_{k}(x)\right|<\varepsilon / k$, where $Y_{k}:=p^{-1}\left(Y_{0, k}\right)$. Since $X_{0}$ is a Stein manifold, we may assume without loss of generality that each $\bar{Y}_{0, k}$ is holomorphically convex. Then there is a strictly pseudoconvex open neighbourhood $D_{0, k} \Subset X_{0}$ of $\bar{Y}_{0, k}, k \geq 1$ (see, e.g., [35]). Since restriction $\left.f\right|_{\bar{D}_{k}} \in$ $\mathcal{A}_{\mathfrak{a}}\left(D_{k}\right), D_{k}:=p^{-1}\left(D_{0, k}\right)$, by Theorem 12.1 there exist functions $h_{k}^{\prime} \in \mathcal{A}_{0}\left(D_{k}\right)$ such that $\sup _{x \in D_{k}}\left|f(x)-h_{k}^{\prime}(x)\right|<\varepsilon /(2 k), k \geq 1$. By the definition of space $\mathcal{A}_{0}\left(D_{k}\right)$, there exists $\iota_{k} \in I$ such that $h_{k}^{\prime} \in \mathcal{A}_{\iota_{k}}\left(D_{k}\right)$. Now, by Proposition 12.2, there exists $h_{k} \in \mathcal{O}_{\mathfrak{a}_{\iota_{k}}}(X)$ such that $\sup _{x \in Y_{k}}\left|h_{k}^{\prime}(x)-h_{k}(x)\right|<\varepsilon /(2 k)$. Thus, $\sup _{x \in Y_{k}}\left|f(x)-h_{k}(x)\right|^{k}<\varepsilon / k$. Since $\mathcal{O}_{\mathfrak{a}_{\iota_{k}}}(X) \subset \mathcal{O}_{0}(X)$, this implies the required result modulo Theorem 12.1 .

Proof of Theorem 12.1. By $\mathcal{A}\left(D_{0}, C_{\mathfrak{a}} X_{0}\right)$ and $\mathcal{A}\left(D_{0}, C_{\mathfrak{a}_{\iota}} X_{0}\right)$ we denote spaces of sections of bundles $\left.C_{\mathfrak{a}} X_{0}\right|_{\bar{D}_{0}}$ and $\left.C_{\mathfrak{a}_{\iota}} X_{0}\right|_{\bar{D}_{0}}$ continuous over $\bar{D}_{0}$ and holomorphic on $D_{0}$. We equip $\mathcal{A}\left(D_{0}, C_{\mathfrak{a}} X_{0}\right)$ with norm $\|f\|:=\sup _{x \in \bar{D}_{0}}\|f(x)\|_{\mathfrak{a}}$ which makes it a Banach space. Then $\mathcal{A}\left(D_{0}, C_{\mathfrak{a}_{\iota}} X_{0}\right)$ is a closed subspace of $\mathcal{A}\left(D_{0}, C_{\mathfrak{a}} X_{0}\right)$. Also, we define linear space

$$
\mathcal{A}_{0}\left(D_{0}, C_{\mathfrak{a}} X_{0}\right):=\bigcup_{\iota \in I} \mathcal{A}\left(D_{0}, C_{\mathfrak{a}_{\iota}} X_{0}\right)
$$

We have natural isomorphisms of vector spaces defined similarly to that of Proposition 2.24:

$$
\mathcal{A}_{\mathfrak{a}_{\iota}}(D) \cong \mathcal{A}\left(D_{0}, C_{\mathfrak{a}_{\iota}} X_{0}\right), \quad \mathcal{A}_{0}(D) \stackrel{\cong}{\rightarrow} \mathcal{A}_{0}\left(D_{0}, C_{\mathfrak{a}} X_{0}\right)
$$

(the proof is analogous to the proof of Proposition 2.24). In view of (12.4), Theorem 12.1 can be restated as follows:

(*) Suppose that $X_{0}$ is a Stein manifold and $D_{0} \Subset X_{0}$ is a strictly pseudoconvex subdomain. Then $\mathcal{A}_{0}\left(D_{0}, C_{\mathfrak{a}} X_{0}\right)$ is dense in $\mathcal{A}\left(D_{0}, C_{\mathfrak{a}} X_{0}\right)$.

For the proof of this claim we need the following results.

As before, we define

$$
\mathcal{A}\left(D_{0}, B\right):=\mathcal{O}\left(D_{0}, B\right) \cap C\left(\bar{D}_{0}, B\right)
$$

and endow this space with norm $\|f\|_{\bar{D}_{0}}:=\sup _{x \in \bar{D}_{0}}\|f(x)\|_{B}$. The next result follows easily from a similar result in [35] (in case $B=\mathbb{C}$ ) since all integral formulas and estimates used in its proof are preserved when passing to Banach-valued forms. 
Lemma 12.3. Let $K \subset \mathcal{A}\left(D_{0}, B\right)$ be compact. For every $\varepsilon>0$ there exist an open neighbourhood $D_{0}^{\prime} \Subset X_{0}$ of $\bar{D}_{0}$ and a bounded linear operator $A_{K, \varepsilon}=A_{D_{0}, K, \varepsilon} \in$ $\mathcal{L}\left(\mathcal{A}\left(D_{0}, B\right), \mathcal{A}\left(D_{0}^{\prime}, B\right)\right)$ such that $\left\|f-\left.A f\right|_{\bar{D}_{0}}\right\|_{\bar{D}_{0}}<\varepsilon$ for each $f \in K$.

We prove assertion $(*)$ in three steps.

(1) Let $f \in \mathcal{A}\left(D_{0}, C_{\mathfrak{a}} X_{0}\right)$. Using the construction of Subsection 2.5 .1 (cf. the proof of Theorem 2.28) and Lemma 12.3, we may assume without loss of generality that $f=\left.f^{\prime}\right|_{\bar{D}_{0}}$, where $f^{\prime} \in \mathcal{O}\left(D_{0}^{\prime}, C_{\mathfrak{a}} X_{0}\right)$ and $D_{0}^{\prime} \Subset X_{0}$ is an open neighbourhood of $\bar{D}_{0}$.

We have to show that for every $\varepsilon>0$ there exists a section $F \in \mathcal{A}_{0}\left(D_{0}, C_{\mathfrak{a}} X_{0}\right)$ such that $\sup _{x \in \bar{D}_{0}}\|f(x)-F(x)\|_{\mathfrak{a}}<\varepsilon$.

(2) Let $\mathcal{U}=\left\{U_{k}\right\}_{k=1}^{M}$, where each $U_{k} \Subset D_{0}^{\prime}$ is open biholomorphic to a polydisk in $\mathbb{C}^{n}$, and $D_{0} \Subset \cup_{k=1}^{M} U_{k}$.

Lemma 12.4. For every $\varepsilon>0$ there exist a subspace $\mathfrak{a}_{\iota_{\varepsilon}} \subset \mathfrak{a}\left(\iota_{\varepsilon} \in I\right)$ and sections $F_{\varepsilon, k} \in \mathcal{A}\left(U_{k}, C_{\mathfrak{a}_{\iota \varepsilon}} X_{0}\right)$ such that

$$
\left\|f^{\prime}(x)-F_{\varepsilon, k}(x)\right\|_{\mathfrak{a}}<\varepsilon \quad \text { for all } x \in U_{k}, \quad 1 \leq k \leq M
$$

Proof. Since each $U_{k}, 1 \leq k \leq M$, is simply connected, the bundles $C_{\mathfrak{a}} X_{0}$ and $C_{\mathfrak{a}_{\iota}} X_{0}$ $(\iota \in I)$ admit holomorphic trivializations over $U_{k}$. Using these trivializations we identify sections of these bundles over $U_{k}$ with $\mathfrak{a}$-valued and $\mathfrak{a}_{\iota}$-valued functions on $U_{k}$.

By our assumption, for every $1 \leq k \leq M$ there exists a biholomorphism between $U_{k}$ and an open polydisk $\Delta \subset \mathbb{C}^{n}$ centered at 0 . Without loss of generality we may assume that $f_{k}^{\prime}:=\left.f^{\prime}\right|_{U_{k}}$ is defined over an open neighbourhood of $\bar{\Delta}$. Then $f_{k}^{\prime}$ can be identified by means of the corresponding holomorphic trivialization of bundle $C_{\mathfrak{a}} X_{0}$ with a holomorphic a-valued function defined on an open neighbourhood of $\bar{\Delta}$.

For a given function $h \in \mathcal{O}(\Delta, \mathfrak{a})$ by $T_{0}^{N} h$ we denote its Taylor polynomial of degree $N$ at $x=0$. Choose $N$ so large that

$$
\left\|f_{k}^{\prime}(x)-T_{0}^{N} f_{k}^{\prime}(x)\right\|_{\mathfrak{a}}<\frac{\varepsilon}{2} \quad \text { for all } x \in \Delta, \quad 1 \leq k \leq M,
$$

where $T_{0}^{N} f_{k}^{\prime}(x):=\sum_{|\alpha| \leq N} a_{k, \alpha} x^{\alpha}, a_{k, \alpha} \in \mathfrak{a}$, and $\alpha$ is a multi-index. Since $\mathfrak{a}_{0}$ is dense in $\mathfrak{a}$, for every $\delta>0$ and all $1 \leq k \leq M,|\alpha| \leq N$, there exist $a_{k, \alpha}^{\varepsilon} \in \mathfrak{a}_{0}$ such that $\left\|a_{k, \alpha}-a_{k, \alpha}^{\varepsilon}\right\|_{\mathfrak{a}}<\delta$. We choose $\delta>0$ to be sufficiently small so that

$$
\sup _{x \in \Delta}\left\|\sum_{|\alpha| \leq N} a_{k, \alpha} x^{\alpha}-F_{\varepsilon, k}(x)\right\|_{\mathfrak{a}}<\frac{\varepsilon}{2},
$$

where $F_{\varepsilon, k}(x):=\sum_{|\alpha| \leq N} a_{k, \alpha}^{\varepsilon} x^{\alpha}$. Therefore,

$$
\left\|f_{k}^{\prime}(x)-F_{\varepsilon, k}(x)\right\|_{\mathfrak{a}}<\varepsilon \quad \text { for all } x \in \Delta, \quad 1 \leq k \leq M .
$$

By definition, there exists $\iota_{\varepsilon} \in I$ such that $\mathfrak{a}_{\iota_{\varepsilon}}$ contains all $a_{k, \alpha}^{\varepsilon}(1 \leq k \leq M$, $|\alpha| \leq N)$; hence $F_{\varepsilon, k} \in \mathcal{A}\left(\Delta, \mathfrak{a}_{\iota_{\varepsilon}}\right)$. 
(3) We also need the following result.

Lemma 12.5. In notation of Lemma 12.4, for every $\varepsilon>0$ there exists a section $F \in \mathcal{A}\left(D_{0}, C_{\mathfrak{a}_{\iota \varepsilon}} X_{0}\right) \subset \mathcal{A}_{0}\left(D_{0}, C_{\mathfrak{a}} X_{0}\right)$ such that

$$
\left\|F(x)-F_{\varepsilon, k}(x)\right\|_{\mathfrak{a}}<C \varepsilon \quad \text { for all } x \in U_{k} \cap \bar{D}_{0}, \quad 1 \leq k \leq M,
$$

for some $C>0$ independent of section $f^{\prime} \in \mathcal{A}_{0}\left(D_{0}^{\prime}, C_{\mathfrak{a}} X_{0}\right)$ and $\varepsilon>0$.

Proof. There exists an open neighbourhood $D_{0}^{\prime \prime} \Subset D_{0}^{\prime}$ of $\bar{D}_{0}$ such that $D_{0}^{\prime \prime} \Subset$ $\cup_{k=1}^{M} U_{k}$. We may assume without loss of generality that $D_{0}^{\prime \prime}$ is strictly pseudoconvex. Let $B$ be a complex space. By $\Lambda_{b}^{(0, q)}\left(D_{0}^{\prime \prime}, B\right), q \geq 0$, we denote the space of bounded continuous $B$-valued $(0, q)$-forms on $D_{0}^{\prime \prime}$ endowed with norm $\|\cdot\|_{D_{0}^{\prime \prime}, B}^{(0, q)}$ defined by formula (6.1) with respect to local coordinates on the cover $\left\{U_{k}\right\}_{k=1}^{M}$ of $D_{0}^{\prime \prime}$.

Next, we define a holomorphic 1-cocycle as follows. If $U_{k} \cap U_{l} \neq \varnothing$, then

$$
g_{k l}:=\left.F_{\varepsilon, k}\right|_{U_{k} \cap U_{l} \cap D_{0}^{\prime \prime}}-\left.F_{\varepsilon, l}\right|_{U_{k} \cap U_{l} \cap D_{0}^{\prime \prime}} \in \mathcal{A}\left(U_{k} \cap U_{l} \cap D_{0}^{\prime \prime}, C_{\mathfrak{a}_{\iota \varepsilon}} X_{0}\right),
$$

and $g_{k l}:=0$ if $U_{k} \cap U_{l} \cap D_{0}^{\prime \prime}=\varnothing$.

Let $\left\{\rho_{k}\right\}_{k=1}^{M} \subset C^{\infty}\left(X_{0}\right)$ be a collection of nonnegative functions such that $\operatorname{supp}\left(\rho_{k}\right) \Subset U_{k}, 1 \leq k \leq M$, and $\sum_{k=1}^{m} \rho_{k} \equiv 1$ on $\bar{D}_{0}^{\prime \prime}$.

We set $\tilde{g}_{l}:=\sum_{k=1}^{M} \rho_{k} g_{k l} \in C^{\infty}\left(U_{l} \cap D_{0}^{\prime \prime}\right)$ so that $g_{k l}=\tilde{g}_{k}-\tilde{g}_{l}$ on $U_{k} \cap U_{l} \cap D_{0}^{\prime \prime}$. Then the family $\left\{\bar{\partial} \tilde{g}_{l}\right\}$ determines a $\bar{\partial}$-closed $(0,1)$-form $\omega$ on $D_{0}^{\prime \prime}, \omega:=\bar{\partial} \tilde{g}_{l}$ on $U_{l} \cap D_{0}^{\prime \prime}$, taking values in bundle $C_{\mathfrak{a}_{\iota \varepsilon}} X_{0}$.

Recall (Subsection 2.5) that since $X_{0}$ is a Stein manifold, there exists a holomorphic Banach vector bundle $E$ such that $C_{\mathfrak{a}_{\iota \varepsilon}} X_{0} \oplus E=X_{0} \times B$ for some Banach space $B$. By $q: X_{0} \times B \rightarrow C_{\mathfrak{a}_{\iota \varepsilon}} X_{0}$ and $i: C_{\mathfrak{a}} X_{0} \rightarrow X_{0} \times B, q \circ i=\mathrm{Id}$, we denote the corresponding bundle morphisms.

Let $\tilde{\omega}:=i(\omega) \in \Lambda_{b}^{(0,1)}\left(D_{0}^{\prime \prime}, B\right)$. Since $q$ is a holomorphic bundle morphism, $\tilde{\omega}$ is $\bar{\partial}$-closed. Moreover, according to Lemma 12.4 ,

$$
\sup _{x \in U_{k} \cap U_{l} \cap D_{0}^{\prime \prime}}\left\|g_{k l}(x)\right\|_{\mathfrak{a}}<2 \varepsilon \quad \text { for all } k, l .
$$

Therefore by the construction of $\omega$ and by continuity of $i$ and the fact that $D_{0}^{\prime \prime} \Subset X_{0}$ we obtain that for some $c>0$ independent of $\omega$,

$$
\|\tilde{\omega}\|_{D_{0}^{\prime \prime}, B}^{(0,1)} \leq c \varepsilon .
$$

Then by Lemma 6.1 there exists a function $\tilde{\eta} \in \Lambda_{b}^{0,0}\left(D_{0}^{\prime \prime}, B\right)$ such that $\bar{\partial} \tilde{\eta}=\tilde{\omega}$ and

$$
\|\tilde{\eta}\|_{D_{0}^{\prime \prime}, B}^{(0,0)} \leq C_{1}\|\tilde{\omega}\|_{D_{0}^{\prime \prime}, B}^{(0,1)} \leq C_{1} c \varepsilon
$$

for some $C_{1}>0$ independent of $\omega$. We set $\eta:=q(\tilde{\eta}) \in C^{1}\left(D_{0}^{\prime \prime}, C_{\mathfrak{a}_{\iota \varepsilon}} X_{0}\right)$. Since $q$ is a holomorphic bundle morphism and $D_{0}^{\prime \prime} \Subset X_{0}$,

$$
\bar{\partial} \eta=\omega \quad \text { and } \quad \sup _{x \in D_{0}^{\prime \prime}}\|\eta(x)\|_{\mathfrak{a}} \leq C_{2} C_{1} c \varepsilon
$$

for some $C_{2}>0$ independent of $\omega$. 
Since $D_{0} \Subset D_{0}^{\prime \prime}$, the restriction $\left.\eta\right|_{\bar{D}_{0}}$ is continuous on $\bar{D}_{0}$. We define

$$
\left.F\right|_{U_{k} \cap \bar{D}_{0}}:=\left.F_{\varepsilon, k}\right|_{U_{k} \cap \bar{D}_{0}}-\left.\tilde{g}_{k}\right|_{U_{k} \cap \bar{D}_{0}}+\left.\eta\right|_{U_{k} \cap \bar{D}_{0}}, \quad 1 \leq k \leq M .
$$

It follows that $F \in \mathcal{A}\left(D_{0}, C_{\mathfrak{a}_{\llcorner\varepsilon}} X_{0}\right)$ and

$$
\sup _{x \in \bar{D}_{0}}\left\|F-F_{\varepsilon, k}\right\|_{\mathfrak{a}} \leq 2 M \varepsilon+C_{2} C_{1} c \varepsilon=: C \varepsilon,
$$

as required. This completes the proof of the lemma.

Assertion $(*)$ now follows from Lemmas 12.4 and 12.5. The proof of Theorem 12.1 is complete.

\section{Proofs of Theorems 5.10, 5.18 and Lemmas 5.13, 5.14}

\subsection{Proof of Theorem $\mathbf{5 . 1 0}$}

We will use notation and results of Subsection 5.1.

By definition, every point in $Y$ has a neighbourhood $V \subset Y$ over which, for every $N \geq 1$, there exists a free resolution

$$
\left.\left.\left.\left.\mathcal{O}_{Y}^{m_{4 N}}\right|_{V} \stackrel{\varphi_{4 N-1}}{\longrightarrow} \cdots \stackrel{\varphi_{2}}{\longrightarrow} \mathcal{O}_{Y}^{m_{2}}\right|_{V} \stackrel{\varphi_{1}}{\longrightarrow} \mathcal{O}_{Y}^{m_{1}}\right|_{V} \stackrel{\varphi_{0}}{\longrightarrow} \mathcal{A}\right|_{V} \longrightarrow 0 .
$$

We need to show that sheaf $\tilde{\mathcal{A}}$ is coherent on $c_{\mathfrak{a}} X$, i.e., that every point $x \in c_{\mathfrak{a}} X$ has a neighbourhood $U \subset c_{\mathfrak{a}} X$ over which sheaf $\left.\tilde{\mathcal{A}}\right|_{U}$ has free resolutions of any finite length. If $x \in c_{\mathfrak{a}} X \backslash Y$, then we can choose $U$ such that $U \cap Y=\varnothing$; hence, $\left.\tilde{\mathcal{A}}\right|_{U}=0$ trivially has free resolutions of any finite length. Now, let $x \in Y$. Shrinking $V$, if necessary, and applying Proposition 5.4 we can choose $U \ni x$ such that $V=Y \cap U$ and there exists a biholomorphism that maps $U$ onto $U_{0} \times K$, where $U_{0} \subset X_{0}$ is biholomorphic to an open polydisk in $\mathbb{C}^{n}, K \subset \hat{G}_{\mathfrak{a}}$ is open, and $V$ is mapped onto $V_{0} \times K$, where $V_{0} \subset U_{0}$ is a complex submanifold. Thus, applying this biholomorphism we may assume that

$$
U=U_{0} \times K, \quad V=V_{0} \times K .
$$

We will need the following.

Lemma 13.1. The trivial extension $\widetilde{\mathcal{O}_{Y}}$ of $\mathcal{O}_{Y}$ has free resolutions of any finite length over $U$.

Proof. By definition, $\left.\widetilde{\mathcal{O}_{Y}}\right|_{U}$ is isomorphic to the quotient sheaf $\mathcal{O}_{U} / I_{V}$, where $\mathcal{O}_{U}:=\left.\mathcal{O}\right|_{U}$ is the sheaf of germs of holomorphic functions on $U, I_{V} \subset \mathcal{O}_{U}$ is the ideal sheaf of $V \subset U$, i.e., we have an exact sequence

$$
\left.0 \rightarrow I_{V} \rightarrow \mathcal{O}_{U} \rightarrow \widetilde{\mathcal{O}_{Y}}\right|_{U} \rightarrow 0 .
$$

Following the argument of the proof of Proposition 5.5, we obtain that sheaf $I_{V}$ has free resolutions of any finite length over $U$. Then using a free resolution of $I_{V}$ over $U$ of length $N$, we extend (13.2) to a free resolution of $\left.\overline{\mathcal{O}_{Y}}\right|_{U}$ of length $N+1$. Since $N$ was chosen arbitrarily, this completes the proof. 
Now we finish the proof of Theorem 5.10. Since sequence (13.1) is exact, the corresponding sequence of trivial extensions

$$
\left.\left.\left.\left.\widetilde{\mathcal{O}}_{Y}^{m_{4 N}}\right|_{U} \stackrel{\tilde{\varphi}_{4 N-1}}{\longrightarrow} \cdots \stackrel{\tilde{\varphi}_{2}}{\longrightarrow} \widetilde{\mathcal{O}}_{Y}^{m_{2}}\right|_{U} \stackrel{\tilde{\varphi}_{1}}{\longrightarrow} \widetilde{\mathcal{O}}_{Y}^{m_{1}}\right|_{U} \stackrel{\tilde{\varphi}_{0}}{\longrightarrow} \tilde{\mathcal{A}}\right|_{U} \longrightarrow 0
$$

is also exact; here $\tilde{\varphi}_{i}:=e \circ \varphi_{i} \circ r_{U, V}$, where $r_{U, V}:\left.\left.\widetilde{\mathcal{O}}_{Y}\right|_{U} \rightarrow \mathcal{O}_{Y}\right|_{V}$ is the restriction homomorphism, and $e: \mathcal{B} \rightarrow \tilde{\mathcal{B}}$ is the canonical homomorphism that maps an analytic sheaf $\mathcal{B}$ on $V$ to its trivial extension $\tilde{\mathcal{B}}$ on $U$.

By Lemmas 7.15 and 7.17 in [15] sequence (13.1) truncated to the $N$-th term is completely exact over $V$ (i.e., the corresponding sequence of sections is exact, see Definition 7.22 in [15]), therefore sequence (13.3) truncated to the $N$-th term is completely exact as well. Since by Lemma 13.1 each sheaf $\widetilde{\mathcal{O}}_{Y}^{m_{i}}$ in (13.3) has free resolutions over $U$ of any finite length, Lemma 9.3 in [15] implies that $\left.\tilde{\mathcal{A}}\right|_{U}$ has free resolutions over $U$ of any finite length as well. This implies that sheaf $\tilde{\mathcal{A}}$ is coherent on $c_{\mathfrak{a}} X$.

\subsection{Proof of Lemma 5.13}

We use the following consequence of Theorem 4.6 in [15]:

Lemma 13.2. Let $V_{0,1}, V_{0,2} \subset \mathbb{C}^{n}$ be open and connected and $K_{1}, K_{2} \subset \hat{G}_{\mathfrak{a}}$ be open. A map $F \in \mathcal{O}\left(V_{0,1} \times K_{1}, V_{0,2} \times K_{2}\right)$ admits presentation

$$
F(z, \omega)=\left(f_{\omega}(z), h(\omega)\right), \quad(z, \omega) \in V_{0,1} \times K_{1}
$$

where $f_{\omega} \in \mathcal{O}\left(V_{0,1}, V_{0,2}\right)$ depend continuously on $\omega \in K_{1}$ and $h \in C\left(K_{1}, K_{2}\right)$.

Proof. Let $\pi^{1}: V_{0,2} \times K_{2} \rightarrow V_{0,2}, \pi^{2}: V_{0,2} \times K_{2} \rightarrow K_{2}$ be the natural projections. By Theorem 4.6 in [15], $\left(\pi^{2} \circ F\right)(\cdot, \omega) \equiv$ const for all $\omega \in K_{1}$. Thus, we define $h \in C\left(K_{1}, K_{2}\right)$ as $h(\omega):=\left(\pi^{2} \circ F\right)(\cdot, \omega), \omega \in K_{1}$, and $f_{\omega}(z):=\left(\pi^{1} \circ F\right)(z, \omega)$, $(z, \omega) \in V_{0,1} \times K_{1}$.

Now, suppose $\varphi_{i} \in \mathcal{O}\left(V, V_{i} \times K_{i}\right)$, where $V_{i} \subset \mathbb{C}^{n}$ are open and connected, $K_{i} \subset \hat{G}_{\mathfrak{a}}$ are open $(i=1,2)$, are coordinate maps of an open subset $V \subset Y$. Let $F:=\varphi_{2} \circ \varphi_{1}^{-1}$. By the above lemma, $F(z, \omega)=\left(f_{\omega}(z), h(\omega)\right)\left((z, \omega) \in V_{1} \times K_{1}\right)$, where $f_{\omega} \in \mathcal{O}\left(V_{1}, V_{2}\right)$ depend continuously on $\omega$ and $h \in C\left(K_{1}, K_{2}\right)$. Since $F$ is a biholomorphism, $h: K_{1} \rightarrow K_{2}$ is a homeomorphism. Then replacing $F$, if necessary, by the holomorphic map $G \circ F$, where $G(z, \omega):=\left(z, h^{-1}(w)\right),(z, \omega) \in$ $V_{2} \times K_{2}$, we may assume without loss of generality that $K_{2}=K_{1}=: K$ and that $h=\mathrm{Id}$.

In order to prove the lemma it suffices to show that for each $p \in C^{\infty}\left(V_{0,2} \times K\right)$ its pullback $F^{*} p \in C^{\infty}\left(V_{0,1} \times K\right)$. Indeed, we have $\left(F^{*} p\right)(z, \omega)=p\left(f_{\omega}(z), \omega\right)$. Since $V_{1} \ni z \mapsto f .(z)=F(z, \cdot)$ is a holomorphic and, hence, a $C^{\infty}$ function taking values in the Fréchet space $C(K)$, the required result follows by the chain rule. 


\subsection{Proof of Lemma 5.14}

(For similar arguments, see [11].)

Lemma 13.3. For an open cover $\mathcal{U}=\left\{U_{\gamma}\right\}$ of $Y$ there exists an open cover $\mathcal{V}=\left\{\hat{\Pi}\left(V_{0, \alpha}, K_{\alpha, \beta}\right): V_{0, \alpha} \subset X_{0}, K_{\alpha, \beta} \subset \hat{G}_{\mathfrak{a}}\right.$ are open $\}$ of $c_{\mathfrak{a}} X$ such that $\left\{V_{0, \alpha}\right\}$ is a locally finite open cover of $X_{0}$, for each $\alpha$ the number of distinct sets $K_{\alpha, \beta}$ is finite and $\cup_{\beta} \hat{\Pi}\left(V_{0, \alpha}, K_{\alpha, \beta}\right)=\bar{p}^{-1}\left(V_{0, \alpha}\right)$, and $\left\{Y \cap \hat{\Pi}\left(V_{0, \alpha}, K_{\alpha, \beta}\right)\right\}$ is a refinement of $\mathcal{U}$.

Proof. By the definition of the relative topology of $Y$, there exists a collection $\tilde{\mathcal{U}}=\left\{\tilde{U}_{\gamma}\right\}$ of open subsets of $c_{\mathfrak{a}} X$ such that $U_{\gamma}=\tilde{U}_{\gamma} \cap Y$ for all $\gamma$. Further, since $Y \subset c_{\mathfrak{a}} X$ is closed, $\tilde{\mathcal{U}} \cup\left\{c_{\mathfrak{a}} X \backslash Y\right\}$ is an open cover of $c_{\mathfrak{a}} X$. By the definition of topology on $c_{\mathfrak{a}} X$ the latter cover admits a refinement by sets of the form $\hat{\Pi}\left(V_{0}, K\right)$, where $V_{0} \subset X_{0}, K \subset \hat{G}_{\mathfrak{a}}$ are open. Since $c_{\mathfrak{a}} X$ has compact fibres, we may choose this refinement $\left\{\hat{\Pi}\left(V_{0, \alpha}, K_{\alpha, \beta}\right)\right\}$ so that $\left\{V_{0, \alpha}\right\}$ is a locally finite open cover of $X_{0}$ and for each $\alpha$ the number of distinct sets $K_{\alpha, \beta}$ is finite and $\cup_{\beta} \hat{\Pi}\left(V_{0, \alpha}, K_{\alpha, \beta}\right)=$ $\bar{p}^{-1}\left(V_{0, \alpha}\right)$. By our construction, $\left\{Y \cap \hat{\Pi}\left(V_{0, \alpha}, K_{\alpha, \beta}\right)\right\}$ is a refinement of $\mathcal{U}$.

The open cover $\mathcal{V}$ introduced in the lemma admits a subordinate partition of unity $\left\{\nu_{\alpha, \beta}\right\}, \nu_{\alpha, \beta}:=\bar{p}^{*} \rho_{\alpha} \cdot \pi_{\alpha}^{*} \mu_{\alpha, \beta}$, where $\left\{\rho_{\alpha}\right\} \subset C^{\infty}\left(V_{0, \alpha}\right)$ is a partition of unity subordinate to cover $\left\{V_{0, \alpha}\right\}$ of $X_{0},\left\{\mu_{\alpha, \beta}\right\} \subset C\left(\bar{p}^{-1}\left(x_{\alpha}\right)\right), x_{\alpha} \in V_{0, \alpha}$ is fixed, is a partition of unity subordinate to cover $\left\{\hat{\Pi}\left(V_{0, \alpha}, K_{\alpha, \beta}\right) \cap \bar{p}^{-1}\left(x_{\alpha}\right)\right\}_{\beta}$ of $\bar{p}^{-1}\left(x_{\alpha}\right)$ and $\pi_{\alpha}: \bar{p}^{-1}\left(V_{0, \alpha}\right) \rightarrow \bar{p}^{-1}\left(x_{\alpha}\right)$ is the continuous projection defined in local coordinates $(x, \omega)$ on $\bar{p}^{-1}\left(V_{0, \alpha}\right)\left(\cong V_{0, \alpha} \times \hat{G}_{\mathfrak{a}}\right)$ as $\pi_{\alpha}(x, \omega):=\left(x_{\alpha}, \omega\right)$. By definition $\nu_{\alpha, \beta} \in C^{\infty}\left(\hat{\Pi}\left(V_{0, \alpha}, K_{\alpha, \beta}\right)\right)$; hence, the restriction of $\left\{\nu_{\alpha, \beta}\right\}$ to $Y$ is a $C^{\infty}$ partition of unity subordinate to $\mathcal{U}$ (see Subsection 5.5).

\subsection{Proof of Theorem $\mathbf{5 . 1 8}$}

Lemma 13.4. Let $U_{0} \subset X_{0}, K \subset \hat{G}_{\mathfrak{a}}$ be open, $f \in \mathcal{O}\left(U_{0} \times K\right)$ be such that $\nabla_{z} f(z, \eta) \neq 0$ for all $(z, \eta) \in Z_{f}:=\left\{(z, \eta) \in U_{0} \times K: f(z, \eta)=0\right\}$.

If $g \in \mathcal{O}\left(U_{0} \times K\right)$ vanishes on $Z_{f}$, then $h:=g / f \in \mathcal{O}\left(U_{0} \times K\right)$.

(The proof follows straightforwardly from Proposition 5.4.)

Proof of Theorem 5.18. By Proposition 5.1, $M_{X}$ is homeomorphic to the maximal ideal space of $\mathcal{O}\left(c_{\mathfrak{a}} X\right)$. It follows from Theorem 2.10 that algebra $\mathcal{O}\left(c_{\mathfrak{a}} X\right)$ separates points of $c_{\mathfrak{a}} X$, therefore we have a continuous injection $c_{\mathfrak{a}} X \hookrightarrow M_{X}$ defined via point evaluation homomorphisms. Let us show that this map is surjective.

The transpose to the pullback homomorphism $\bar{p}^{*}: \mathcal{O}\left(X_{0}\right) \rightarrow \mathcal{O}\left(c_{\mathfrak{a}} X\right)$ is a map $\bar{p}_{*}: M_{X} \rightarrow M_{X_{0}}$, where the latter is the maximal ideal space of algebra $\mathcal{O}\left(X_{0}\right)$. Since $X_{0}$ is Stein, $M_{X_{0}}$ can be naturally identified with $X_{0}$ (see, e.g., [34]) so that $\left.\bar{p}_{*}\right|_{c_{\mathfrak{a}} X}=\bar{p}$. Hence, for $\varphi \in M_{X}$ there exists a point $x_{0} \in X_{0}$ such that $\bar{p}_{*}(\varphi)=\delta_{x_{0}}$, the evaluation homomorphism at point $x_{0}$.

Next, there exists a function $h \in \mathcal{O}\left(X_{0}\right)$ such that $X_{0}^{n-1}:=\left\{x \in X_{0}: h(x)=0\right\}$, $n:=\operatorname{dim}_{\mathbb{C}} X$, is a non-singular complex hypersurface, $d h(z) \neq 0$ for each $z \in X_{0}^{n-1}$, 
and $x_{0} \in X_{0}^{n-1}$, see [25]. We set $X^{n-1}:=p^{-1}\left(X_{0}^{n-1}\right)$ and $c_{\mathfrak{a}} X^{n-1}:=\bar{p}^{-1}\left(X_{0}^{n-1}\right)$. Now, if $f \in \mathcal{O}\left(c_{\mathfrak{a}} X\right)$ is identically zero on $c_{\mathfrak{a}} X^{n-1}$, then $\varphi(f)=0$. Indeed, by Lemma 13.4, the function $\tilde{f}:=f / \bar{p}^{*} h \in \mathcal{O}\left(c_{\mathfrak{a}} X\right)$, hence,

$$
\varphi(f)=\varphi(\tilde{f}) \varphi\left(\bar{p}^{*} h\right)=\varphi(\tilde{f}) \delta_{x_{0}}(h)=0 .
$$

Thus, there exists a homomorphism $\varphi_{1}$ of the quotient algebra $\mathcal{O}\left(c_{\mathfrak{a}} X\right) / I_{c_{\mathfrak{a}} X^{n-1}}$, where $I_{c_{\mathfrak{a}} X^{n-1}}$ is the ideal of holomorphic functions in $\mathcal{O}\left(c_{\mathfrak{a}} X\right)$ vanishing on $c_{\mathfrak{a}} X^{n-1}$, such that $\varphi=\varphi_{1} \circ q_{1}$, where $q_{1}: \mathcal{O}\left(c_{\mathfrak{a}} X\right) \rightarrow \mathcal{O}\left(c_{\mathfrak{a}} X\right) / I_{c_{\mathfrak{a}} X^{n-1}}$ is the quotient homomorphism. According to Theorem 2.10, we have a natural isomorphism

$$
\mathcal{O}\left(c_{\mathfrak{a}} X\right) / I_{c_{\mathfrak{a}} X^{n-1}} \cong \mathcal{O}\left(c_{\mathfrak{a}} X^{n-1}\right)
$$

defined by restrictions of functions in $\mathcal{O}\left(c_{\mathfrak{a}} X\right)$ to $c_{\mathfrak{a}} X^{n-1}$; hence $\varphi_{1}$ can be identified with an element of the maximal ideal space of algebra $\mathcal{O}\left(c_{\mathfrak{a}} X^{n-1}\right)$.

Starting with $c_{\mathfrak{a}} X^{n-1}$ instead of $c_{\mathfrak{a}} X$ we proceed similarly to define flags of complex submanifolds $X_{0}^{k} \subset X_{0}, X^{k} \subset X, c_{\mathfrak{a}} X^{k} \subset c_{\mathfrak{a}} X$ of codimension $n-k$ and homomorphisms $\varphi_{n-k}: \mathcal{O}\left(c_{\mathfrak{a}} X^{k}\right) \rightarrow \mathbb{C}$ such that $\varphi_{n-k-1}=\varphi_{n-k} \circ q_{n-k}(0 \leq k \leq$ $n-1)$, where $q_{n-k}: \mathcal{O}\left(c_{\mathfrak{a}} X^{k+1}\right) \rightarrow \mathcal{O}\left(c_{\mathfrak{a}} X^{k+1}\right) / I_{c_{\mathfrak{a}} X^{k}}=\mathcal{O}\left(c_{\mathfrak{a}} X^{k}\right)$ are the quotient homomorphisms.

By the definition, $\varphi_{n}$ is an element of the maximal ideal space of algebra $\mathcal{O}\left(c_{\mathfrak{a}} X^{0}\right)$, where $X_{0}^{0}=\left\{x_{0}, x_{1}, \ldots\right\}$ is a discrete set. Clearly, $\mathcal{O}\left(c_{\mathfrak{a}} X^{0}\right) \cong \sqcup_{i \geq 0}$ $C\left(\bar{p}^{-1}\left(x_{i}\right)\right)$. Moreover, if $f \in I_{x_{0}} \subset \mathcal{O}\left(c_{\mathfrak{a}} X^{0}\right)$, the ideal of functions vanishing on $\bar{p}^{-1}\left(x_{0}\right)$, then $f=f \cdot \bar{p}^{*} g_{x_{0}}$, where $g_{x_{0}} \in \mathcal{O}\left(X_{0}^{0}\right), g_{x_{0}}\left(x_{i}\right)=1-\delta_{0 i}$ (Kronecker delta), so that

$$
\varphi_{n}(f)=\varphi_{n}(f) \varphi_{n}\left(\bar{p}^{*} g_{x_{0}}\right)=\varphi_{n}(f) \varphi\left(\bar{p}^{*} g\right)=\varphi_{n}(f) g_{x_{0}}\left(x_{0}\right)=0
$$

here $g \in \mathcal{O}\left(X_{0}\right)$ is such that $\left.g\right|_{X_{0}^{0}}=g_{x_{0}}$.

Thus, there exists a homomorphism

$$
\varphi_{n+1}: \mathcal{O}\left(c_{\mathfrak{a}} X^{0}\right) / I_{x_{0}}=C\left(\bar{p}^{-1}\left(x_{0}\right)\right) \rightarrow \mathbb{C}
$$

such that $\varphi_{n}=\varphi_{n+1} \circ q_{n+1}$, where $q_{n+1}: \mathcal{O}\left(c_{\mathfrak{a}} X^{0}\right) \rightarrow C\left(\bar{p}^{-1}\left(x_{0}\right)\right)$ is the quotient homomorphism. Since $\bar{p}^{-1}\left(x_{0}\right)$ is compact Hausdorff, the maximal ideal space of $C\left(\bar{p}^{-1}\left(x_{0}\right)\right)$ is homeomorphic to $\bar{p}^{-1}\left(x_{0}\right)$. In particular, $\varphi_{n+1}=\delta_{\omega}$ for some $\omega \in \bar{p}^{-1}\left(x_{0}\right)$.

Finally, we have

$$
\varphi(f)=\varphi_{n+1}\left(\left.f\right|_{\bar{p}^{-1}\left(x_{0}\right)}\right)=f(\omega)=\delta_{\omega}(f), \quad f \in \mathcal{O}\left(c_{\mathfrak{a}} X\right)
$$

as required. This shows that the natural map $c_{\mathfrak{a}} X \rightarrow M_{X}$ is a continuous bijection. It is easily seen that this map is a homeomorphism since $c_{\mathfrak{a}} X$ is locally compact and $X_{0} \rightarrow M_{X_{0}}$ is a homeomorphism.

The proof of the theorem is complete. 


\section{References}

[1] Besicovitch, A. S.: Almost periodic functions. Dover, New York, 1955.

[2] Bohr, H.: Almost periodic functions. Chelsea Publishing Co., New York, 1947.

[3] Boggess, A.: CR manifolds and the tangential Cauchy-Riemann complex. Studies in Advanced Mathematics, CRC Press, Boca Raton, FL, 1991.

[4] Bredon, G. E.: Sheaf theory. Graduate texts in Mathematics 170, Springer-Verlag, New York, 1997.

[5] Brudnyi, A.: Projections in the space $H^{\infty}$ and the corona theorem for coverings of bordered Riemann surfaces. Ark. Mat. 42 (2004), no. 1, 31-59.

[6] BRudnyi, A.: On holomorphic functions of slow growth on coverings of strongly pseudoconvex manifolds. J. Funct. Anal. 249 (2007), no. 2, 354-371.

[7] Brudnyi, A.: Hartogs type theorems on coverings of Stein manifolds. Internat. J. Math. 17 (2006), no. 3, 339-349.

[8] Brudnyi, A.: Representation of holomorphic functions on coverings of pseudoconvex domains in Stein manifolds via integral formulas on these domains. J. Funct. Anal. 231 (2006), no. 2, 418-437.

[9] Brudnyi, A.: Holomorphic $L^{p}$-functions on coverings of strongly pseudoconvex manifolds. Proc. Amer. Math. Soc. 137 (2009), no. 1, 227-234.

[10] Brudnyi, A.: Grauert- and Lax-Halmos-type theorems and extension of matrices with entries in $H^{\infty}$. J. Funct. Anal. 206 (2006), 87-108.

[11] Brudnyi, A.: Banach-valued holomorphic functions on the maximal ideal space of $H^{\infty}$. Invent. Math. 193 (2013), no. 1, 187-227.

[12] BRudnyi, A.: Holomorphic Banach vector bundles on the maximal ideal space of $H^{\infty}$ and the operator corona problem of Sz.-Nagy. Adv. Math. 232 (2013), $121-141$.

[13] Brudnyi, A. and Kinzebulatov, D.: Holomorphic almost periodic functions on coverings of complex manifolds. New York J. Math. 17a (2011), 267-300.

[14] Brudnyi, A. And Kinzebulatov, D.: Holomorphic semi-almost periodic functions. Integr. Equ. Operat. Theory 66 (2010), no. 3, 293-325.

[15] Brudnyi, A. And Kinzebulatov, D.: Towards Oka-Cartan theory for algebras of holomorphic functions on coverings of Stein manifolds I. Rev. Mat. Iberoam. 31 (2015), no. 3, 989-1032.

[16] Bungart, L.: Holomorphic functions with values in locally convex spaces and applications to integral formulas. Trans. Amer. Math. Soc. 111 (1964), 317-344; Errata, ibid. 113 (1964), 547.

[17] Demailly, J. P.: Complex analytic and differential geometry. Grenoble, 2009.

[18] Favorov, S.: Zeros of holomorphic almost periodic functions. J. Anal. Math. 84 (2001), 51-66.

[19] Favorov, S.: Almost periodic divisors, holomorphic functions, and holomorphic mappings. Bull. Sci. Math. 127 (2003), no. 10, 859-883.

[20] Favorov, S. and Rashkovskit, A.: Holomorphic almost periodic functions. Acta Appl. Math. 65 (2001), no. 1-3, 217-235.

[21] Favorov, S., Rashkovskit, A. And Ronkin L.: Almost periodic divisors in a strip. J. Anal. Math. 74 (1998), 325-345. 
[22] Favorov, S., Rashkovskit, A. And Ronkin L.: Almost periodic currents and holomorphic chains. C. R. Acad. Sci. Paris Sèr. I Math. 327 (1998), no. 5, 445-449.

[23] Freudenthal, H.: Über die Enden topologischer Räume und Gruppen. Math. Z. 33 (1931), no. 1, 692-713.

[24] Forster, O.: Lectures on Riemann surfaces. Graduates Texts in Mathematics 81, Springer-Verlag, New York-Berlin, 1981.

[25] Forstnerič, F.: Noncritical holomorphic functions on Stein manifolds. Acta Math. 191 (2003), no. 2, 143-189.

[26] Forstnerič, F.: Stein manifolds and holomorphic mappings. The homotopy principle in complex analysis. A Series of Modern Surveys in Mathematics 56, Springer, Heidelberg, 2011.

[27] Gamelin, T.: Uniform algebras. Prentice-Hall, Englewood Cliffs, NJ, 1969.

[28] Gleason, A.: The abstract theorem of Cauchy-Weil. Pacific J. Math. 12 (1962), 511-525.

[29] Grauert, H. And Remmert, R.: Theory of Stein spaces. Die Grundlehren der Mathematischen Wissenschaften 236, Springer-Verlag, Berlin-New York, 1979.

[30] Griffiths, P. And Harris, J.: Principles of algebraic geometry. Wiley Classics Library, John Wiley \& Sons, New York, 1994.

[31] Grigoryan, S.: Generalized analytic functions. Russian Math. Surveys 49 (1994), no. 2, 1-40.

[32] Gromov, M., Henkin G. And Shubin M.: Holomorphic $L^{2}$ functions on coverings of pseudoconvex manifolds. Geom. Funct. Anal. 8 (1998), no. 3, 552-585.

[33] Gunning, R.: Introduction to holomorphic functions of several variables. III. Homological theory. The Wadsworth \& Brooks/Cole Mathematics Series, Wadsworth \& Brooks/Cole Advanced Books \& Software, Monterey, CA, 1990.

[34] Gunning, R. And Rossi, H.: Analytic functions of several complex variables. Prentice-Hall, Englewood Cliffs, NJ, 1965.

[35] Henkin, G. And Leiterer, J.: Theory of functions on complex manifolds. Monographs in Mathematics 79, Birkhäuser Verlag, Basel, 1984.

[36] Harvey, R. And Lawson, G.: On boundaries of complex analytic varieties. I. Ann. of Math. (2) 102 (1975), no. 2, 223-290.

[37] Hirzebruch, F.: Topological methods in algebraic geometry. Die Grundlehren der Mathematischen Wissenschaften 131, Springer-Verlag, New York, 1966.

[38] Jessen, B. And Tornehave, H.: Mean motions and zeros of almost periodic functions. Acta Math. 77 (1945), 137-279.

[39] Kantorovich, L. V. And Akilov, G. P.: Functional analysis. Pergamon Press, Oxford-Elmsford, NY, 1982.

[40] LÁrusson, F.: Holomorphic functions of slow growth on nested covering spaces of compact manifolds. Canad. J. Math. 52 (2000), no. 5, 982-998.

[41] Leiterer, J.: Banach coherent analytic Fréchet sheaves. Math. Nachr. 85 (1978), 91-109.

[42] Lempert, L. and Patyi, I.: Analytic sheaves in Banach spaces. Ann. Sci. École Norm. Sup. (4) 40 (2007), no. 3, 453-486.

[43] Levin, B.: Distribution of zeros of entire functions. Translations of Mathematical Monographs 5, American Mathematical Society, Providence, RI, 1980. 
[44] Lin, V.: Liouville coverings of complex spaces, and amenable groups. Math. USSRSb. 60 (1988), no. 1, 197-216.

[45] Lyons, T. And Sullivan, D.: Function theory, random paths and covering spaces. J. Differential Geom. 19 (1984), no. 2, 299-323.

[46] Mank, W.: Eine neue Definition der fastperiodischen Funktionen. Abh. Math. Sem. Univ. Hamburg 11 (1935), no. 1, 240-244.

[47] von Neumann, J.: Almost periodic functions in a group. I. Trans. Amer. Math. Soc. 36 (1934), no. 3, 445-492.

[48] Ronkin, L.: Jessen's theorem for holomorphic almost periodic functions in tube domains. Sibirskii Mat. Zh. 28 (1987), no. 3, 199-204.

[49] Sarason, D.: Toeplitz operators with semi-almost periodic symbols. Duke Math J. 44 (1977), no. 2, 357-364.

[50] Shabat, B.: Introduction to complex analysis. Part II. Functions of several variables. Translations of Mathematical Monographs 110, American Mathematical Society, Providence, RI, 1992.

[51] Shubin, M.: Almost periodic functions and partial differential operators. Russian Math. Surveys 33 (1978), 1-52.

[52] Tornehave, H.: On the zeros of entire almost periodic function. Math. Fys. Medd. Danske Vid. Selsk. 42 (1989), no. 3, 125-142.

[53] Vesentini, E.: Holomorphic almost periodic functions and positive-definite functions on Siegel domains. Ann. Mat. Pura Appl. (4) 102 (1975), 177-202.

[54] Weil, A.: L'integration dans les groupes topologiques et ses applications. Actual. Sci. Ind. 869, Hermann, Paris, 1940.

[55] Zaidenberg, M., Krein, S. G., Kuchment, P. And Pankov, A.: Banach bundles and linear operators. Russian Math. Surveys 30 (1975), 115-175.

Received September 24, 2013; revised November 12, 2013.

Alexander Brudnyi: Department of Mathematics and Statistics, University of Calgary, 2500 University Drive NW, Calgary, Alberta T2N 1N4, Canada.

E-mail: abrudnyi@ucalgary.ca

Damir Kinzebulatov: The Fields Institute, 222 College Street, Toronto, Ontario M5T 3J1, Canada.

E-mail: damir.kinzebulatov@utoronto.ca 